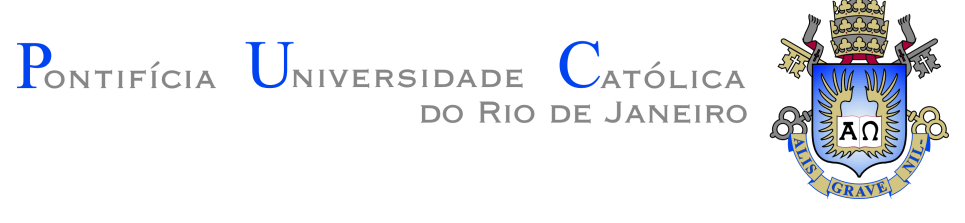

Carolina Moreira Goloni Marques

\title{
Analysis and application of point scanning and full-field optical techniques in vibration detection
}

Tese de Doutorado

Thesis presented to the Programa de Pós-graduação em Engenharia Elétrica of PUC-Rio in partial fulfillment of the requirements for the degree of Doutor em Ciências - Engenharia Elétrica.

Advisor : Prof. Guilherme Penello Temporão Co-Advisor:

Dr. Olivier Jacquin

Co-Advisor:

Dr. Eric Lacot 

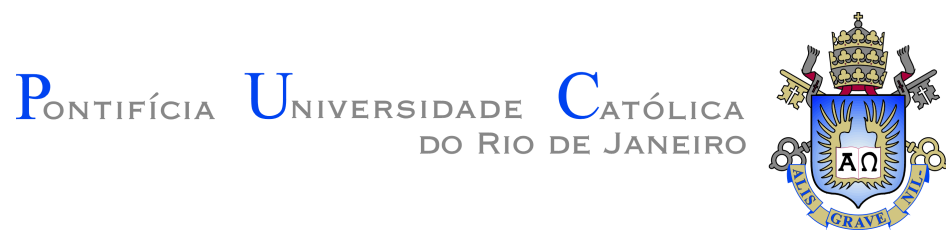

\section{Carolina Moreira Goloni Marques}

\section{Analysis and application of point scanning and full-field optical techniques in vibration \\ detection}

Thesis presented to the Programa de Pós-graduação em Engenharia Elétrica of the Departamento de Engenharia Elétrica do Centro Técnico Científico da PUC-Rio as partial fulfillment of the requirements for the degree of Doutor.

Prof. Guilherme Penello Temporão

Advisor

Centro de Estudos em Telecomunicações - PUC-Rio

Prof. Jean Pierre von de Weid

Centro de Estudos em Telecomunicações - PUC-Rio

Prof. Giancarlo Vilela de Faria Ouro Negro SA

Dr. Rogério Passy

MLS Wireless

Dr. Andrew Henry Cordes

Centro de Estudos em Telecomunicações - PUC-Rio

Dra. lakyra Borrakuens Couceiro Inmetro

Prof. Marcio da Silveira Carvalho Coordinator of the

Centro Técnico Científico - PUC-Rio

Rio de Janeiro, November 29th, 2016 
All rights reserved

\section{Carolina Moreira Goloni Marques}

The author got her bachelor's degree from PUC-Rio in Computer Engineering. Then obtained a master's degree also from PUC-Rio in Electrical Engineering, on optolectronic systems. As part of the doctorate, she collaborated on a project with the Laboratoire Interdisciplinaire de Physique (LIPhy) at the Université Grenoble Alpes, in France.

Bibliographic data

Marques, Carolina Moreira Goloni

Analysis and application of point scanning and full-field optical techniques in vibration detection / Carolina Moreira Goloni Marques; advisor: Guilherme Penello Temporão; coadvisores: Olivier Jacquin, Eric Lacot. - 2016.

v., $101 \mathrm{f}$ : il. color ; $30 \mathrm{~cm}$

1. Tese (doutorado) - Pontifícia Universidade Católica do Rio de Janeiro, Departamento de Engenharia Elétrica, 2016.

Inclui bibliografia.

1. Engenharia Elétrica - Teses. 2. Imageamento Fotoacústico;. 3. LOFI;. 4. Interferometria;. 5. Vibrometria.. I. Temporão, Guilherme Penello. II. Jacquin, Olivier. III. Lacot, Eric. IV. Pontifícia Universidade Católica do Rio de Janeiro. Departamento de Engenharia Elétrica. V. Título. 


\section{Acknowledgements}

To my advisors Professors Guilherme Temporão, Olivier Jacquin and Eric Lacot for their support on the realization of this work.

To the CNPq and the PUC-Rio, for the financial support, without which this work would not have been realized.

To my family, for their never ending incentive throughout this process and during my time in France, especially to Théo without whom none of this would have been possible.

To the OPTIMA group at LIPhy for welcoming me into their lab and the constant help with my adaptation. And to Vadim Girardeau, with whom I had the pleasure of working closely for the past two years.

To my friends in Grenoble and in Rio, who offered me various forms of support, but most importantly, their friendship.

And a special thank you to Joseph, for sharing his extensive knowledge and skills with kindness and enthusiasm, but also for the great support and optimism. 


\section{Abstract}

Marques, Carolina Moreira Goloni; Temporão, Guilherme Penello (Advisor) ; Jacquin, Olivier (Co-Advisor); Lacot, Eric. Analysis and application of point scanning and full-field optical techniques in vibration detection. Rio de Janeiro, 2016. 101p. Tese de Doutorado - Departmento de Engenharia Elétrica, Pontifícia Universidade Católica do Rio de Janeiro.

Photoacoustic imaging combines aspects of optical and ultrasound imaging and, consequently, combines some of their particular advantages: imaging of specific tissue components in a depth up to several centimeters becomes possible with a high image contrast. The most straightforward strategy to produce photoacoustic images is point-wise excitation and detection in a scanning mode. The main focus of this work is to analyze and implement optical techniques to detect ultrasound vibrations in a photoacoustic system. We evaluate different interferometric systems, both point scanning and full field. Two techniques that were implemented and further discussed are the Laser Optical Feedback Imaging (LOFI), which is a sensitive, point scanning, heterodyne interferometer that uses the dynamics of the laser, and a full field, stroboscopic Mach-Zehnder to acquire ultrasound vibration measurements. We discuss the results from each system and its advantages with the intended application.

\section{Keywords}

Photoacoustic Imaging; LOFI; Interferometry; Vibrometry. 


\section{Resumo}

Marques, Carolina Moreira Goloni; Temporão, Guilherme Penello; Jacquin, Olivier; Lacot, Eric. Análise e aplicação de técnicas ópticas de mapeamento pontual e campo pleno na detecção de vibração. Rio de Janeiro, 2016. 101p. Tese de Doutorado - Departamento de Engenharia Elétrica, Pontifícia Universidade Católica do Rio de Janeiro.

A maioria dos métodos de avaliação de ondas vibratórias é realizada através do contato entre o sensor e o objeto a ser analisado. Esse requisito de contato impõe algumas limitações de quais objetos podem ser avaliados, como por exemplo, o tamanho do mesmo. Uma técnica que expandiu a capacidade de medição se comparado aos sensores tradicionais de vibração é a vibrometria laser doppler. Ela possibilita medições não intrusivas com alta resolução espacial e tempo de teste reduzido. Dentre as várias aplicações que se beneficiam das aquisições com o vibrômetro laser doppler, o imageamento fotoacústico se encontra no centro do objetivo deste trabalho. O imageamento fotoacústico é uma tecnologia emergente que supera o alto espalhamento óptico em amostras biológicas utilizando o efeito fotoacústico. Ele combina aspectos de imageamento óptico e ultrassom e, consequentemente, algumas de suas vantagens: imagear certos componentes biológicos em profundidades de alguns centímetros e com alto contraste. A técnica mais direta de obter imagens em um sistema fotoacústico é a excitação pontual da amostra e a detecção por varredura. O foco deste trabalho é analisar e implementar uma técnica óptica para detecção das vibrações ultrassonoras em um sistema fotoacústico. Foram avaliados diferentes sistemas interferométricos experimentais, tanto por mapeamento pontual quanto por campo pleno. O princípio da interferometria e a teoria das medições quantitativas são apresentados para explicar as técnicas experimentais utilizadas para aquisição das ondas vibratórias. Igualmente, a técnica de processamento do sinal vibratório é discutida. Dentre as técnicas testadas experimentalmente, duas foram implementadas, analisadas e discutidas em detalhe e seus resultados foram apresentados e comparados entre si. A primeira técnica discutida é o Laser Optical Feedback Imaging (LOFI), um interferômetro heteródino que utiliza a dinâmica do laser e a sua grande sensibilidade ao fenômeno de reinjeção óptica para realizar medições de alta precisão do sinal retroespalhado da amostra. Essa técnica realiza suas aquisições ponto a ponto, através do 
do escaneamento do feixe do laser na superfície da amostra. Esse modo de aquisição possui um tempo de teste e processamento elevado, mas com alta resolução espacial e com a possibilidade de medir a região transitória. A segunda técnica utilizada para a aquisição de medidas de vibrações ultrassonoras é o estroboscópio Mach-Zehnder, um interferômetro de campo pleno que possibilita a aquisição do padrão de speckle de toda a região de interesse da amostra com baixo tempo de aquisição e processamento. Dessa forma, essa técnica tem um grande potencial para aplicações em tempo real. Os resultados experimentais de ambos os sistemas são apresentados e discutidos em detalhe. Um transdutor piezoelétrico é utilizado para as medições com os dois sistemas, com as mesmas características de teste. Adicionalmente, são apresentados os resultados com a técnica LOFI quando o ganho não-linear é compensado. Esse tipo de teste não é possível no sistema estroboscópico Mach-Zehnder campo pleno, considerando que apenas o LOFI é capaz de realizar medidas transitórias. Para concluir o trabalho, são discutidos e comparados os principais parâmetros para as técnicas testadas: ruído, potência do laser, resolução espacial, resolução temporal, e tempo de aquisição.

\section{Palavras-chave}

Imageamento Fotoacústico; LOFI; Interferometria; Vibrometria. 


\section{Contents}

1 Introduction 13

2 Quantitative measurement of mechanical micrometer and sub microm$\begin{array}{ll}\text { eter deformations } & \mathbf{1 7}\end{array}$

$\begin{array}{lll}2.1 & \text { Principle of interferometry } & 17\end{array}$

$\begin{array}{lll}\text { 2.1.1 Formulation, interference pattern, visibility and phase information } & 17\end{array}$

2.1.2 Specular versus scattering target and its spatial spectrum 21

2.2 Phase measurements 23

2.2.1 Four-phase shifted images and calculus 23

2.2.2 Continuous phase shifting and Hilbert processing 24

$\begin{array}{ll}2.2 .3 & \text { Phase unwrapping } \\ 2.3\end{array}$

2.3 Deformation information extraction 27

$\begin{array}{lll}2.3 .1 & \text { Two measurements } & 28\end{array}$

3 Optical Methods for Vibrometry $\quad 31$

3.1 Laser Optical Feedback Imaging 31

3.2 Shearography 40

3.2.1 The Shearing Relation 42

3.3 Off-axis holography 44

3.4 Stroboscopic Mach-Zehnder 46

3.4.1 Phase analysis 49

4 Experimental results $\quad \mathbf{5 2}$

4.1 LOFI experimental results $\quad 52$

4.1.1 Vibration measurements at $40 \mathrm{kHz}$

4.1.2 Non-linear gain compensation 56

4.2 Stroboscopic Mach-Zehnder experimental results 59

5 Comparative study $\quad 69$

$\begin{array}{lll}5.1 & \text { Parameters } & 69\end{array}$

$\begin{array}{lll}5.2 & \text { Discussion } & 72\end{array}$

$\begin{array}{lll}6 & \text { Conclusion and perspectives } & 74\end{array}$

$\begin{array}{ll}\text { Bibliography } & 77\end{array}$

A Appendix: Non-linear laser dynamics induced by frequency shifted optical feedback

A.1 Laser with frequency-shifted optical feedback 86

$\begin{array}{lll}\text { A.1.1 Basic equations for vibrometry } & 87\end{array}$

$\begin{array}{lll}\text { A.1.2 Signal processing for vibrometry } & 88\end{array}$

A.1.3 Compensation of the gain of the laser dynamics 89

A.1.3.1 Numerical harmonics vibrations $\quad 90$

A.1.3.2 Numerical transient vibrations 92

A.2 Gain of the nonlinear laser dynamics $\quad 95$ 
A.2.1 Bifurcation analysis

95

A.2.2 Asymptotic solution for the nonlinear gain

96

A.2.3 Gain compensation for vibration measurements 


\section{List of Figures}

1.1 Illustration of the principle for the photoacoustic imaging.

2.1 The variation in intensity as a function of the phase difference between two interfering waves.

$\begin{array}{ll}2.2 \text { Example of an interference pattern. } & 19\end{array}$

2.3 High and low contrast fringes. 20

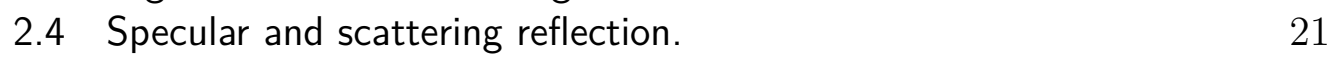

2.5 Fourier Transform of specular and scattering reflection. 22

2.6 Simulation of the spatial spectrum with $f_{0}$ spatial carrier frequency. 24

2.7 Fringe pattern for specular and scattering targets. 25

2.8 2D Spatial spectrum of specular and scattering targets. 25

2.9 Block diagram for the deformation extraction from the phase using two interferograms. 28

$\begin{array}{ll}2.10 \text { Example of an reference interferogram. } & 29\end{array}$

2.11 Fourier Transform of an interferogram with the +1 order selected (on the left) and subsequently filtered and recentered (on the right. 29

2.12 Example phase map of the interferogram of a scattering target a) before (reference) and b) after being deformed.

2.13 Phase difference between the reference and deformed phase maps presented in Figure 2.12.

3.1 LOFI experimental setup.

3.2 $N d^{3+}: Y A G$ microlaser intensity in function of time and its noise spectrum.

3.3 Frequency shift. 34

3.4 Example of an amplitude image recorded by the LOFI. 35

3.5 Example of an phase profile recorded by the LOFI. 36

3.6 Power spectrum of an pumped laser. 39

3.7 Shearography experimental setup. 41

3.8 Result of a shear magnitude calculation using the digital crosscorrelation method.

3.9 Off-axis holography experimental setup.

3.11 Plot of the repeated test for the angle measured in each image by both systems.

3.13 Newport's resolution test target recorded with the system using a non-coherent source.

3.14 Image of a resolution test target recorded with the system using a non-coherent source.

3.15 Fourier spectrum of the interferogram.

4.1 Results for a piezoelectric transducer with a burst of 100 periods at $40 \mathrm{kHz}$. 
4.2 Fourier Transform of the signal with the peaks of the Bessel Function. The green curve shows the linear gain.

4.3 Resulting curve from filtering the +1 order of the Fourier Transform shown in Figure 4.2.

4.4 Resulting 1D filtered signal for one burst at $40 \mathrm{kHz}$.

4.5 Excerpt from the video of the vibrating surface of the piezoelectric sensor.

4.6 Noise spectrum of the LOFI applied to high frequency vibrometry. $\quad 56$

4.7 Detection of a transient-harmonic vibrations. 57

4.8 Piezoelectric transducer analyzed by the Stroboscopic Mach-Zehnder. 59

4.9 Speckle pattern of the piezoelectric transducer's surface. $\quad 60$

4.10 Spatial spectrum of interferometer's beams. 61

4.11 3D plot of a Fourier Transform of one image map. 62

4.12 Resulting curve from filtering the +1 order of the Fourier Transform shown in Figure 4.11.

4.13 3D phase images over a time period.

4.14 Amplitude analysis over time for the 4 different positions defined. 64

4.15 Amplitude of vibration analysis for different voltages applied to the transducer and at different positions.

4.16 Noise analysis with zero voltage applied to the transducer.

4.17 Comparison of amplitude with the transducer running and not running (noise).

4.18 Vibration amplitude at the center of the transducer for 3 different pulse duration.

4.19 Repeatability test.

4.20 Analysis of the repeatability test for the center of the transducer.

5.1 Noise measurements for Stroboscopic Mach-Zehnder (a) and LOFI (b).

A.1 Calculations of a harmonic vibration with a small amplitude of vibration.

A.2 Calculations of a transient vibration with a full time width.

A.3 Calculations of a transient vibration with a full time width.

A.4 Comparison between the complex amplitude of the vibration sidebands obtained from a numerical simulation and the non-linear gain obtained analytically. 


\section{List of Tables}

3.1 LOFI Amplification for different class B lasers. 38

3.2 USAF-1951 resolution target standard specification. 48

5.1 Comparison between LOFI and Mach-Zehnder. 73 


\section{1 \\ Introduction}

Since the first machines were built and the advent of mechanical engineering, we have endeavored to understand the effect of vibrations on such designs to ensure their longevity. For example, before there was a real understanding of modern vibrometry, armies understood the necessity to break step when crossing bridges so as not to destroy them. This was a precaution, due to experience, without actually understanding the phenomenon of resonance (1).

Historically, vibration analysis was first carried out using contact methods. The most simple of these methods was (and still is) the impact hammer motor testing, where a short shock impulse, i.e. a hammer, would be applied to an object and the resulting vibrations would be analyzed by a well trained ear with the help of a stethoscope (2). This method, not being a quantitative evaluation, led to the invention of various vibration sensors for modal analysis. Vibration signals usually consist of several frequencies, which cannot be identified just by analyzing the amplitude-time pattern. The cornerstone of vibration measurements evaluation is the frequency analysis, technique that allows to plot the vibration amplitude against the different frequency components $(3)$.

Most methods for vibration analysis involved contact, which meant physically attaching a sensor to the object to be analyzed (4). This imposed a limitation of which objects could be analyzed, for example, small objects where the mass of the sensor could perturb the natural vibrations of its functioning. For the case of the contact accelerometers, which can be simple to implement and be really small in size (5), they have some major limitations:

- Their intrinsic mass perturbs the vibration measurement unless the mass of the test object is orders of magnitude larger than the mass of the sensor;

○ They have narrow frequency ranges;

- The sensitivity is variable and susceptible to noise; and

o It is not possible to attach them to any object due to physical constraints, for example, targets that are too delicate (e.g. paintings) or too dangerous. 
Despite the advances in technology, with a wide range of sensors, such as electromagnetic, electrostatic, magnetic reluctance, piezoelectric, piezoresistive, potentiometric, capacitance, strain gauge, servo force-balance and motionbalance, and Microelectromechanical systems (MEMS) (6), many objects were still unable to be analyzed. Some of the more prominent examples of non optical vibration measurement tools are capacitive vibration sensors, which present the advantage of the non contact required for measurement. The introduction of LASER and optical vibration analysis changed this scenario.

One development that extended the capabilities of measurements if compared to these traditional vibration sensors is the laser doppler vibrometers (LDV) $(7,8,9)$, as they allow non-intrusive, high spatial resolution measurements with reduced testing time and increased performances (6). It is a precision optical transducer used for determining vibration velocity and displacement at a fixed point (10). Based on the Doppler-effect, the Doppler vibrometer works on the principle of sensing the frequency shift of the backscattered wave from a moving surface by means of an interferometer $(11,12,13)$. Both velocity and displacement can be determined by analyzing the optical interference between a reference beam and the measured beam backscattered from the moving target, which will show a changing optical path length according to its movement. Therefore, the modulation frequency of the interference pattern is proportional to the velocity of the object. As to be able to not only determine the amplitude of the movement but also the direction, the vibrometer works in a heterodyne regime, adding a carrier frequency to one of the beams (14). An acousto-optic modulator or a Bragg cell could be used to generate this frequency shift. A more detailed the description of the principle will be presented at chapter 2 .

Nondestructive testing (NDT) evaluates the properties of a material or system without permanently altering the object of inspection. Hence, Doppler vibrometry is largely applied to NDT and acoustic wave detection. This work focuses on the studies and analysis of high frequency vibrations (from $10 \mathrm{kHz}$ to $5 \mathrm{MHz}$ ), with bi-dimensional and dynamical measurements (15). The main motivation for this work is the acquisition of acoustic waves for photoacoustic imaging.

Photoacoustic imaging is an emerging technology that overcomes the high degree of scattering of optical photons in biological tissue by the use of the photoacoustic effect $(16,17)$. Photoacoustic imaging is based on the emission of pressure waves due to the thermoelastic expansion resulting from the pulsed laser light absorption in a less absorbing environment. Therefore, an excitation wavelength should be chosen accordingly that provides a high 
absorption contrast. The excited pressure waves propagate through the tissue and can be detected at the surface (Figure 1.1).
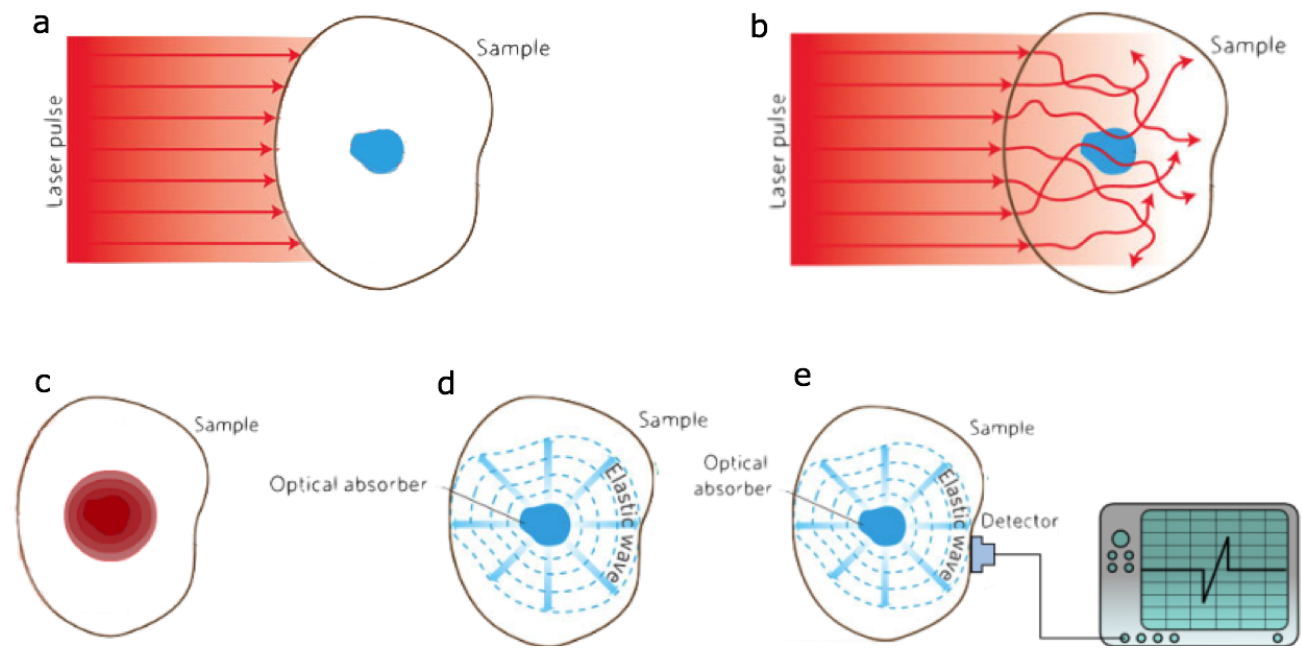

Figure 1.1: Illustration of the principle for the photoacoustic imaging. a. Laser pulse on the sample; b. Optical absorption by the sample; c. Thermal expansion; d. Acoustic waves generated by the thermal expansion; e. Optical detection of the acoustic waves. Based on the figure of (18)

Photoacoustic imaging combines aspects of optical and ultrasound imaging and, consequently, combines some of their particular advantages: imaging of specific tissue components in a depth up to several centimeters becomes possible with a high image contrast. This is an attractive advantage for biomedical imaging, with clinical application varying from skin to brain imaging (19). However, many of the existing approaches share the problem of a relatively high acquisition time, often in the range of minutes, which only allows in vitro or in vivo imaging of anesthetized animals. Furthermore, acoustic contact or, in some cases, total immersion is needed for the sake of impedance matching. The state of science shows a variety of different approaches regarding the recording of the pressure waves $(20,21)$.

The most straightforward strategy to produce photoacoustic images is point-wise excitation and detection in a scanning mode. In order to increase resolution, excitation and detection geometry can be used in a focused mode. Due to the need for scanning both the excitation and detection unit over the object, or the object itself, the time consumption for data acquisition is generally high, e.g., several minutes.

Though the performance of the piezoelectric transducer is still superior to the methods presented in this work, the main purpose for replacing it for an optical detection is its full field, fast acquisition of ultrasound waves with a significant spatial resolution. A thorough description of the measurement of mechanical micrometer and sub micrometer deformations will be detailed on 
chapter 2. It discusses the principle of interferometry, measurements of phase, and the extraction of the deformation information. Chapter 3 will present the different experimental setups assembled to test and assess the applicability of the system to the intended goal: Laser Optical Feedback Imaging (LOFI), shearography (based on a Mach Zehnder interferometer), off-axis holography (based on a Michelson interferometer) and a stroboscopic Mach Zehnder. In chapter 4 , the results of the two prominent systems analyzed for the intended application, the LOFI and the stroboscopic Mach-Zehnder, are presented. Following the presentation of the achieved results for these two techniques, a detailed comparison based on their characteristics is discussed on chapter 5 . To conclude the work and the future perspectives envisioned are discussed at chapter 6 . 


\section{2 \\ Quantitative measurement of mechanical micrometer and sub micrometer deformations}

\section{1}

\section{Principle of interferometry}

\subsection{1}

\section{Formulation, interference pattern, visibility and phase information}

Interferometry is an investigative technique based on the principle of superposition of two or more waves, resulting in a coherent sum of those waves (22). Considering two monochromatic plane waves of equal frequency and polarization represented by the complex wave function:

$$
u(\mathbf{r}, t)=u(\mathbf{r}) \exp (j 2 \pi f t)
$$

where $u(\mathbf{r})=a(\mathbf{r}) \exp (j \phi(\mathbf{r}))$ is the complex amplitude, both depend on the position vector $\mathbf{r}=(x, y, z)$, and $\phi$ is the phase. It is important to point out that both waves are considered linearly polarized and they maintain the same polarization for the following discussion. In case of a difference of polarization between the waves, the interference decreases when the polarization of the waves are non-collinear, disappearing when they become orthogonal $(\pi / 2$ angle).

The superposition principle applies to the sum of waves but, because of the disparity associated with the interference, does not apply to the optical intensity, which is phase dependent. This means that the total intensity of two superposed waves will not be the sum of its intensities. Superposing two waves with complex amplitudes $U_{r}(\mathbf{r})$ and $U_{s}(\mathbf{r})$, the resulting wave has a complex amplitude denoted by:

$$
u(\mathbf{r})=u_{r}(\mathbf{r})+u_{s}(\mathbf{r}) .
$$

As the optical intensity of a wave is $I_{r} \propto\left|u_{r}\right|^{2}$, the total intensity of the resulting superposed wave is the time average of the modulus square of the total complex amplitude: 


$$
\begin{array}{r}
I=\left\langle|u|^{2}\right\rangle_{\text {Tmes }}=\left\langle\left|u_{r}+u_{s}\right|^{2}\right\rangle=\left\langle\left|u_{r}\right|^{2}\right\rangle+ \\
\\
=I_{r}+I_{s}+2 \sqrt{I_{r} I_{s}} \cos \phi
\end{array}
$$

where $\langle\cdot\rangle_{T_{\text {mes }}}$ indicates the time average over a period much longer than $1 / f$ for the measured intensity in $T_{m e s}, I_{r}$ and $I_{s}$ are the intensities of waves $u_{r}$ and $u_{s}$ respectively, and $\phi=\phi_{s}-\phi_{r}$ is the phase difference. This time average is related to the detector, where the exposure time $T_{m e s}$ is of the order of micro and nanosecond on cameras and photodiodes while the wave frequency is of order of hundreds of terahertz. For simplicity, the position vector was suppressed in this equation.

The detected intensity varies sinusoidally with the phase difference between the two waves, as Figure 2.1 represents in 1D the variation of $\phi$ of Equation 2-4. The optical path difference $d$ relates the phase difference with the optical path length between the two wave that have the same frequency $\left(f_{r}=f_{s}\right)$ :

$$
\phi=\phi_{s}-\phi_{r}=\left(\frac{2 \pi}{\lambda}\right)\left(L_{s}-L_{r}\right)=\left(\frac{2 \pi}{\lambda}\right) d,
$$

where $\lambda$ is the wavelength, $L_{r}$ is the reference path and $L_{s}$ is what we call object path, both traveling from the source to the observation plane.

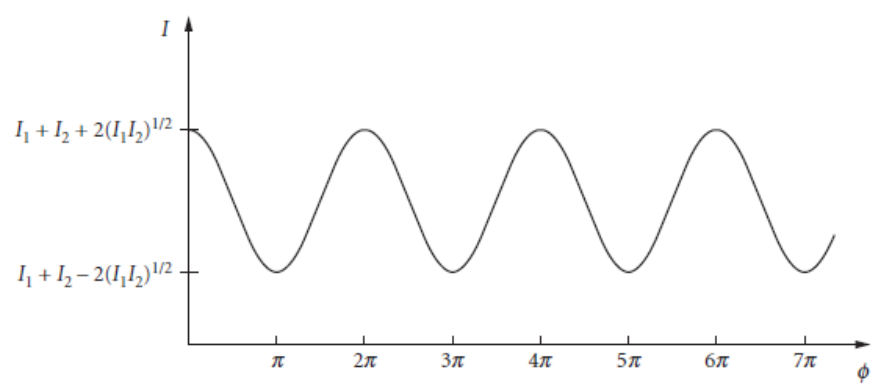

Figure 2.1: The variation in intensity as a function of the phase difference between two interfering waves.

For the case above, there is no shift between the frequencies of the waves. This type of interferometry is know as homodyne. When there is a frequency shift, meaning $f_{r} \neq f_{s}$, it is called heterodyne interferometry. In this case, the phase $\phi$ is described by:

$$
\phi=\frac{2 \pi}{\lambda}(\Delta f+\lambda d)
$$

Varying from a constructive (in phase) to a destructive (out of phase) interference between two Gaussian plane waves, the phase difference will be mapped out as an interference pattern, illustrated by Figure 2.2. The sinusoidal 
variation of the detected intensity will create the interference fringes, where the phase difference is constant along a particular fringe.

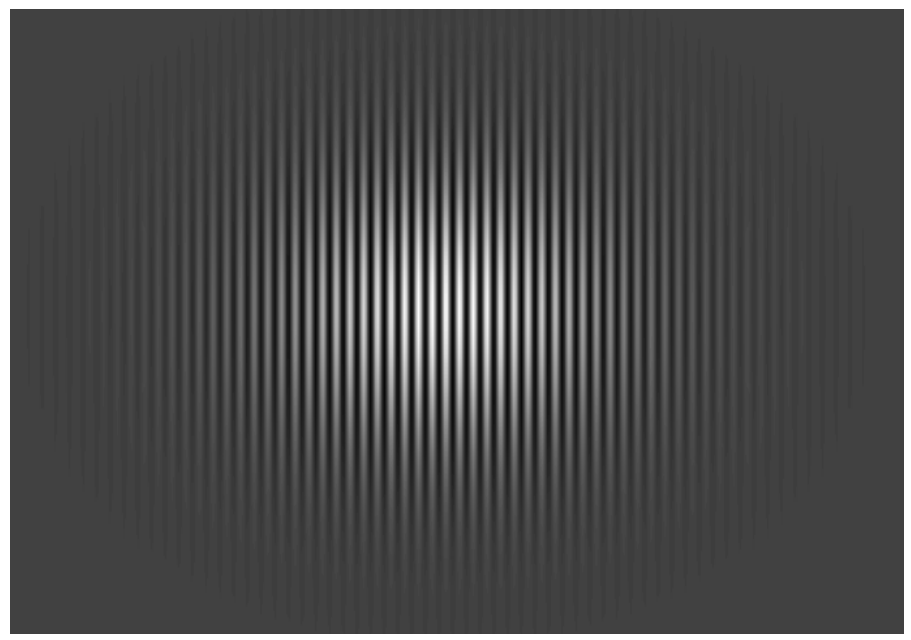

Figure 2.2: Example of an interference pattern. The dark fringes represent the destructive interference while the bright fringes are constructive interference. Along a particular fringe, the phase difference is constant.

Detectors, such as humans eyes, respond to intensity which is the square of the complex amplitude and has the effect of rectifying the waveform. They perceive the time averaged intensity of the rectified waveform, hence the dark and bright areas of the interferogram for out-of-phase and in-phase interference, respectively. Therefore, with the information on the optical path, which is related to the distance compared to the reference wave, it is possible to extract information from the phase, information that is limited by $\lambda$. The precision of the measurements is directly tied to the wavelength.

It can also be noticed in Figure 2.2 that there's a change in intensity throughout the fringe pattern due to a difference in amplitude between the two waves. When the waves don't have the same amplitude, it will compromise both destructive and constructive interference and the fringes won't present optimum contrast, as represented in Figure 2.3, and reduce the maximum amplitude. Therefore, it is clear the importance of approximately achieving equal intensity of the interfering waves. The visibility is defined as:

$$
\gamma=\frac{I_{\max }-I_{\min }}{I_{\max }+I_{\min }}
$$

where $I_{\max }$ is the maximum intensity of the fringe pattern, defined by the sum of the amplitudes of the two interfering beams when they are in phase converted to an intensity and time averaged, and $I_{\text {min }}$ is the minimum intensity, the condition when the two interfering beams are out of phase. The quality 
of the interference pattern is defined by the contrast, aiming to a maximum visibility of 1 .

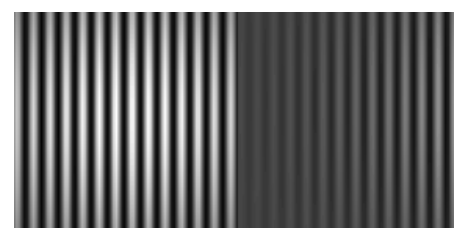

Figure 2.3: High (picture on the left) and low (picture on the right) contrast fringes. The phase varies along $\mathrm{x}$ but not in $\mathrm{y}$, having $\phi=\frac{2 \pi}{\lambda} \alpha x$, where $\alpha$ is the tilting angle.

Considering the visibility, which has values between 0 and 1, Equation 2-4 can be rewritten as:

$$
I=\left(I_{r}+I_{s}\right)\{1+\gamma \cos \phi\}
$$

The maximum visibility occurs when the waves have equal intensity, and these intensities can vary with position, so that the average intensity and fringe visibility can also vary across the fringe pattern. As described, it is possible to acquire from the interference pattern both the information of the intensity difference between the waves (providing the fringe visibility), and the phase information by means of multiple acquisitions, which is related to optical path.

Another factor influencing the contrast is the coherence length. We assumed the light was monochromatic and therefore spatially coherent. Since this does not completely correspond to reality, spatial and temporal coherence will affect the interference between the waves and the its visibility. Two waves are coherent if their wavefronts are correlated in space and in time. It is characterized by its ability to produce observable constructive and destructive interference in a time-averaged basis. However, in certain interferometers, for example the interferometers of amplitude division, the spatial coherence is not as critical.

For heterodyne interferometers, although the frequency of both wave are not the same $\left(\Delta f \ll \frac{1}{T_{m e s}}\right)$, those frequencies must be coherent with the detector.

The observed fringe visibility is a function of the spatial and temporal coherence of the source. The coherence length can be used to quantify the degree of temporal coherence as the propagation length over which coherence degrades significantly. The spatial coherence function relates the interference of these two points independently of other points on the source. 
Chapter 2. Quantitative measurement of mechanical micrometer and sub micrometer deformations

\subsection{2}

\section{Specular versus scattering target and its spatial spectrum}

We can distinguish two types of targets to be analyzed in an optical system: specular targets, which is the case closest to an ideal surface (non rugged surface), e.g. mirrors; and scattering targets, rough surfaces that create what is called speckle patterns.

Considering one ray of the incident light, when a specular surface is illuminated, the light always reflects in the same direction. On the other hand, for a scattering surface we observe what is known as diffuse reflection, when the light reflects and diffuses in many directions, in a random manner. The phase of the light field is randomized in space and the diffraction patterns observed displays a granular pattern called speckle. Figure 2.4 presents the resulting reflection from a specular and a scattering target for a Gaussian laser beam. While we observe a smooth result on the specular surface, the diffuse surface presents a speckle pattern.

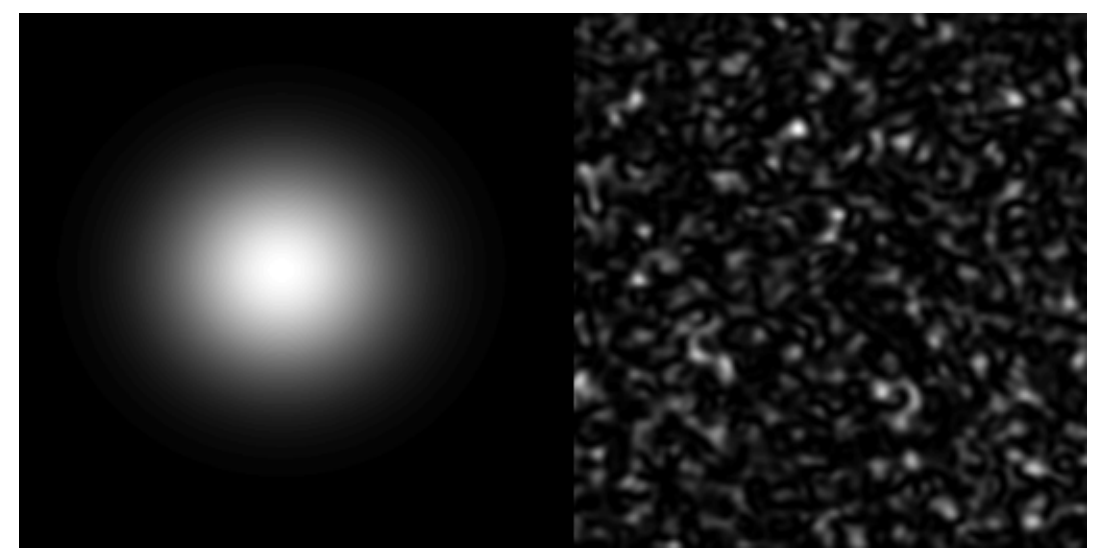

Figure 2.4: Simulations on specular (on the left) and scattering (on the right) reflection of a Gaussian beam.

Figure 2.5 shows the spectrum in $1 \mathrm{D}$ and $2 \mathrm{D}$ of both specular and scattering represented in Figure 2.4. In the frequency domain, the specular Gaussian beam is close to a Dirac delta function while the spectrum of scattering reflection is a random distribution.

When a non specular surface is illuminated with a highly coherent laser light, the phase of the contributions from the many scattering centers is also random. Hence, the speckle pattern influences the resulting interference fringes since by its own consists of a multitude of bright spots where the interference has been highly constructive, dark spots where the interference has been highly destructive (23).

The size of the speckles, a statistical average of the distance between adjacent regions of maximum and minimum brightness, can be given approx- 


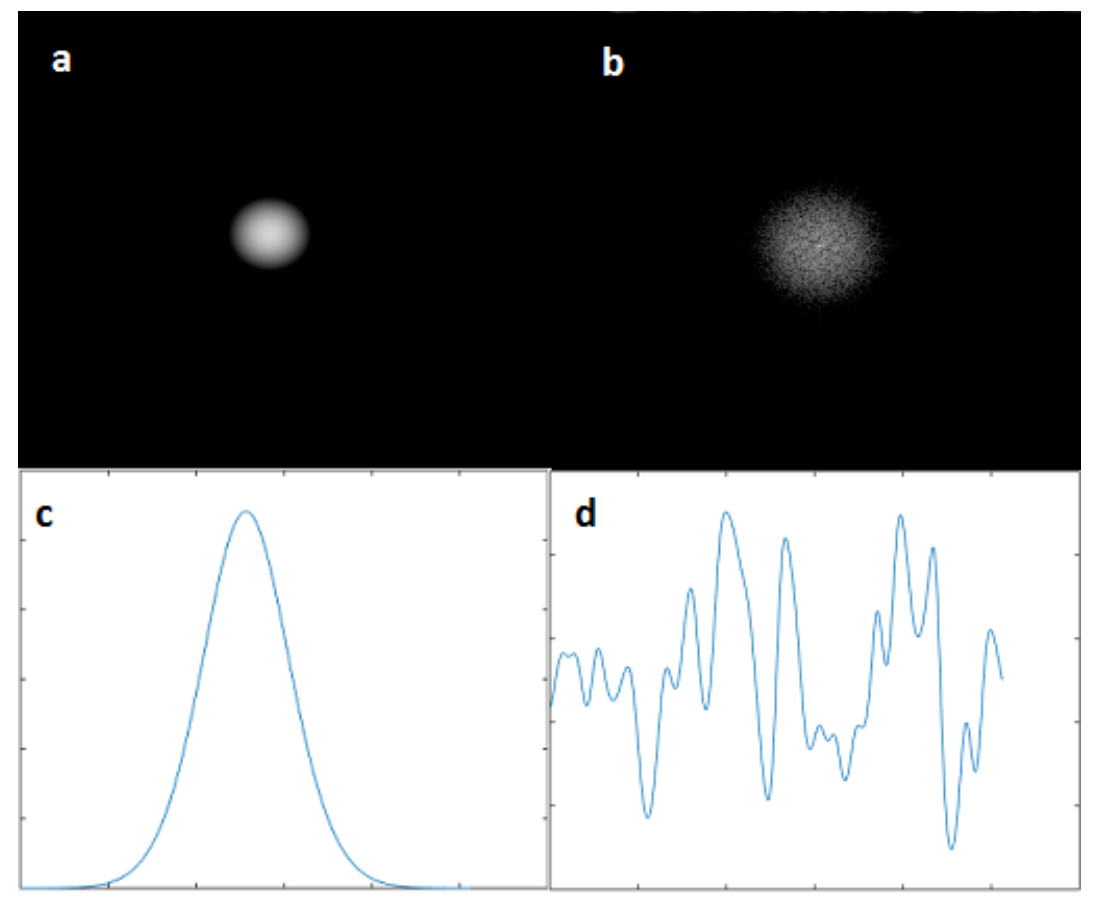

Figure 2.5: 1D and 2D Fourier Transform of the specular (c and a) and the scattering ( $d$ and b) reflection of the Gaussian beams presented in Figure 2.4.

imately for two different cases (24). Speckle patterns are often divided as objective and subjective speckle. Objective speckle is formed naturally when the coherent light illuminates a scattering surface, and its size $\sigma$ is:

$$
\sigma \approx 1.2 \frac{\lambda L}{D}
$$

where $L$ is the distance between the diffuse object and the image plane, and $D$ is the diameter of the region illuminated. Subjective speckle is produced at the image place of a lens and the size of the individual speckles is then related to the numerical aperture of the imaging lens and it is given approximately by:

$$
\sigma \approx 0.6 \frac{\lambda}{N . A .} \approx 1.2(1+M) \lambda F
$$

where $M$ is the magnification factor of the imaging lens and $F$ is the aperture ratio of the lens ( $f$ number). As it will be further discussed in the section describing the experimental systems set up, the relation between the size of the speckle and the aperture of the imaging system play an important part in the analysis of the interference. In the next section, the phase measurement will be discussed and an analysis of the interference of both specular and scattering cases with a reference wave will be presented. 


\section{2}

\section{Phase measurements}

With the theory of interferometry and the fringe pattern formation presented, the main goal is to access the phase information and be able to extract the information intended from the analyzed object. However, in Equation 2.1.1, we are not able to directly access the phase $\phi$. This means some techniques are needed to be able to do so. In this section we present two different methods to extract the phase information.

\subsection{1}

\section{Four-phase shifted images and calculus}

The four-phase shifting measuring technique can determine the shape of a wave by calculating a phase map from the measured intensities. Phaseshifting interferometry requires the acquisition of several images over time, which during this time period, the fringes must be stable (25). The phase difference between the two beams in the interferometer is varied linearly, step by step, with time and the values of intensity at any point in the interference pattern are averaged over a number of equal segments covering one period of the sinusoidal signal. In this method, the intensity values are measured for four known phase increments. Between two consecutive measurements, the phase is incremented by $\alpha_{i}$ and the intensity becomes:

$$
I(x, y)=I_{r}+I_{s}+2 \sqrt{I_{r} I_{s}} \cos \left(\phi+\alpha_{i}\right) .
$$

The minimum number of steps to reconstruct this intensity function is three. One of the most popular phase shifting methods uses four steps, being represented, for examples, as four $\pi / 2$ shifts, as:

$$
\begin{aligned}
& I_{A}=I_{r}+I_{s}+2 \sqrt{I_{r} I_{s}} \cos \phi \\
& I_{B}=I_{r}+I_{s}-2 \sqrt{I_{r} I_{s}} \sin \phi \\
& I_{C}=I_{r}+I_{s}-2 \sqrt{I_{r} I_{s}} \cos \phi \\
& I_{D}=I_{r}+I_{s}+2 \sqrt{I_{r} I_{s}} \sin \phi .
\end{aligned}
$$

The phase difference between the interfering beams is given by the relation:

$$
\phi=\arctan \left(\frac{I_{D}-I_{B}}{I_{A}-I_{C}}\right)
$$

Generally, static fringe analysis is one order of magnitude less precise than phase-shifting interferometry. However, as discussed above, this method requires at least 3 phase steps to construct the phase map and acquire the phase information. Next section presents an alternative to this technique. 


\section{2 .2}

\section{Continuous phase shifting and Hilbert processing}

When the phase shift is made in a continuous manner rather than in steps, the frequency of the shifting beam is permanently modified, and a spatial beating between the two beams is formed. This frequency shift can be added to the system by applying a tilt to the reference mirror of the interferometer. This method is also known as spatial carrier frequency and has the advantage of recording only one interferogram to access the phase information.

$$
I(x, y)=I_{r}+I_{s}+2 \sqrt{I_{r} I_{s}} \cos \left(\phi+2 \pi f_{0} x\right) .
$$

where $f_{0}=\sin \alpha / \lambda$ is the spatial-carrier frequency generated by tilting the reference wave. Applying the Fourier Transform to Equation 2-3,

$$
\mathcal{F}\{I\}=U_{r} \otimes U_{r}^{*}+U_{s} \otimes U_{s}^{*}+U_{r} \otimes U_{s}^{*}+U_{s} \otimes U_{r}^{*}
$$

where $U_{r}=\mathcal{F}\left\{u_{r}\right\}, U_{s}=\mathcal{F}\left\{u_{s}\right\}$, and $\otimes$ represents the convolution operation. With the tilt of angle $\alpha$ between the two waves, we are able to spatially separate the last two convolution terms from each other in Equation 2-18.

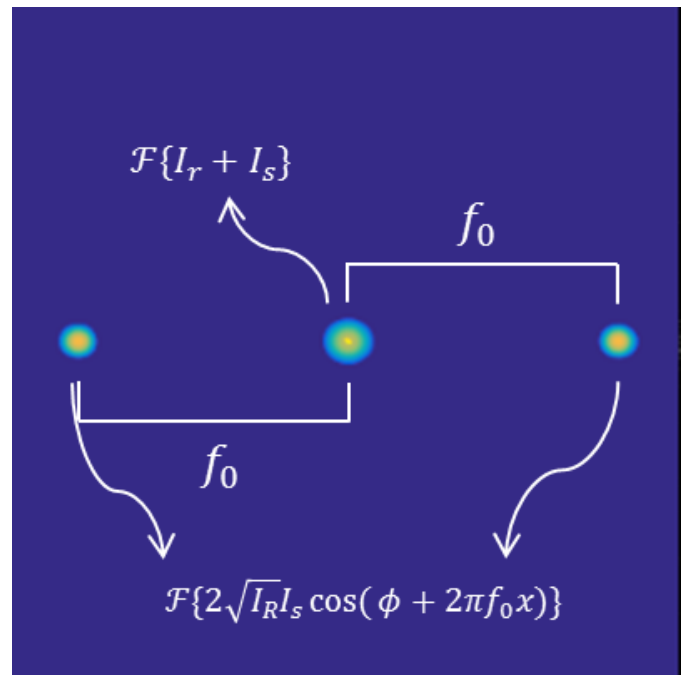

Figure 2.6: Simulation of the spatial spectrum with a $f_{0}$ spatial carrier frequency.

If we interfere the specular and scattering reflected beams presented in Figure 2.4 with a tilted reference wave, the resulting fringe pattern will also present similar differences. Figure 2.7 shows the fringe pattern of the interference of specular beam with a plane wave and the fringe pattern of a scattering target also interfering with a plane wave. The distance between the fringes is influenced by the $\alpha$ angle between the both waves.

When comparing the spatial spectrum of both patterns, as depicted in Figure 2.8, it is easy to identify each case. The distance between the order 


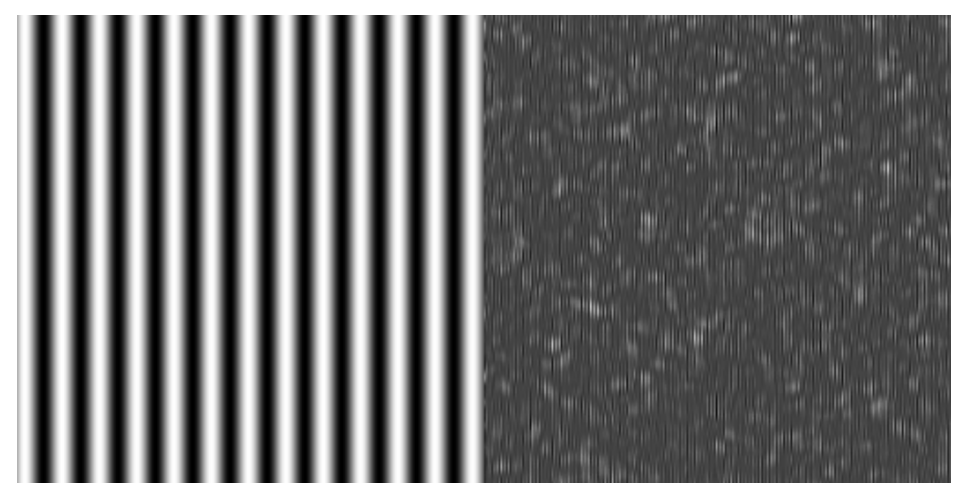

Figure 2.7: Fringe pattern for specular and scattering targets. On the left, interference between two plane waves tilted of $\alpha$ radians. On the right, interference between a plane wave and a wave reflected by diffusing object.

zero and \pm 1 orders are determined $\alpha / \lambda, \alpha$ being the angle created by the tilt added to the reference wave. In both cases, the central order of the resulting Fourier Transform represents the first two terms of Equation 2-18, while the phase information can be extract from the third and forth terms that result in the \pm 1 orders of the FT.

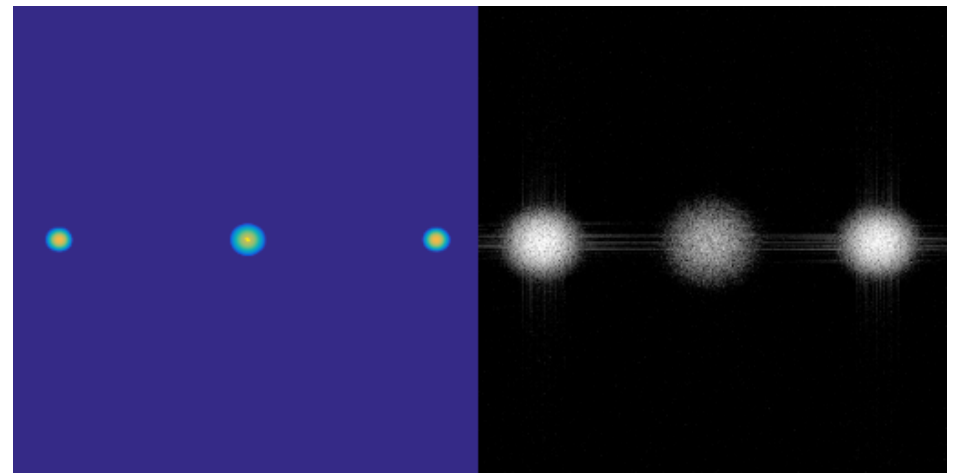

Figure 2.8: Simulation of the spatial spectrum of specular and scattering targets. On the left, the spatial spectrum of two plane waves tilted at $\alpha$ radians and on the right, the spatial spectrum of a diffusing object.

The Hilbert transform is a one dimensional linear operator which takes a function, $v(x)$, and generates another function, $\mathcal{H} v(x)$, in the same domain. It is well known that Hilbert transform can introduce a real signal $\pi / 2$-phase shift and consequently make the imaginary part of complex signal corresponding to the real signal (3). The Hilbert transform of a function $f(x)$ is defined by:

$$
\mathcal{H} v(x)=-\frac{1}{\pi x} \otimes v(x)
$$

Therefore, the Hilbert transform of the original function $v(x)$ represents its harmonic conjugate. In the frequency domain, the Hilbert Transform can be written as 
where

$$
\mathcal{F}\{\mathcal{H} v(x)\}=\mathcal{F}\left\{-\frac{1}{\pi x} \otimes v(x)\right\}=i \operatorname{sgn} s V(s),
$$

$$
\operatorname{sgn}(x)= \begin{cases}+1 & x>0 \\ -1 & x<0\end{cases}
$$

To access the phase $\phi$, we then calculate $\phi$ :

$$
\phi=\arctan \left(\frac{\mathcal{H} v(x)}{V(s)}\right) .
$$

Going back to our interference result, we take $v(x)$ as the interference term $2 \sqrt{I_{R} I_{S} \cos \left(\phi+2 \pi f_{0} x\right)}$. Applying the Hilbert operator $\mathcal{H} v(x)$ will transform the cos into a sin. When trying to access the phase, as indicated by eq. 2-22, we have:

$$
\begin{aligned}
\arctan \left(\frac{\mathcal{H} v(x)}{V(s)}\right) & =\arctan \left(\frac{2 \sqrt{I_{R} I_{S} \sin \left(\phi+2 \pi f_{0} x\right)}}{2 \sqrt{I_{R} I_{S} \cos \left(\phi+2 \pi f_{0} x\right)}}\right) \\
& =\arctan \left(\frac{\sin \left(\phi+2 \pi f_{0} x\right)}{\cos \left(\phi+2 \pi f_{0} x\right)}\right) \\
& =\arctan \left(\tan \left(\phi+2 \pi f_{0} x\right)\right) \\
& =\phi+2 \pi f_{0} x .
\end{aligned}
$$

Despite being a popular tool in signal processing, we decided to take a different approach to access the phase information that would suit better our system. Once we filter our Fourier Transform of the reference and deformed interference pattern, meaning we only keep the order +1 , and recenter it, we apply the inverse Fourier Transform. With this step, we return to the time domain, but now with the filter interference map $\left(\mathcal{F}_{-\infty}\left[\mathcal{F}\left[e^{j \phi}\right]\right]=e^{j \phi}\right)$. This way, to access the phase, we take:

$$
\arg \left(e^{j \phi}\right)=\arctan \left(\frac{\Im\left(e^{j \phi}\right)}{\Re\left(e^{j \phi}\right)}\right)=\arctan \left(\frac{\sin \phi}{\cos \phi}\right)=\arctan (\tan \phi)=\phi .
$$

With this filtering process, we can directly access the phase information. Having the phase map for both reference and deformed cases, we can extract the deformation information, which will be discussed in the following section. The phase discontinuities need to be removed, with the phase unwrapping technique. 


\subsection{3}

\section{Phase unwrapping}

Phase is a property of a real signal related to the temporal and/or spatial wavelength and thus influences the signal only through phase principal values, also called wrapped phase values, where the absolute phase is wrapped into the interval $(-\pi, \pi]$ radians. Therefore, the nonlinear phase unwrapping operation is concerned with obtain an estimate for the phase from the wrapped values (26).

To obtain a continuous phase measurement, $2 \pi$ discontinuities that may appear in the wrapped phase maps need to be removed by the phase unwrapping process. Since the phase is calculated from an arc tangent there are discontinuities in the phase calculation. Normally the arc tangent is defined over a range of $-\pi / 2$ to $\pi / 2$. However, in phase-shifting interferometry we know the signs of the individual sines and cosines. This means that we know which quadrant the phase is in, so when the arc tangent is performed the phase is determined over a range of $-\pi$ to $\pi$.

The range of phase that can be measured can be further extended if we set a limit on the slope of the measured phase such that the phase difference between adjacent detector points is less than $\pi$. The phase difference between adjacent detector points can then be calculated and when the calculated phase difference is greater than $\pi$ we know that the phase difference is calculated too large because of $2 \pi$ ambiguities in the arc tangent. The $2 \pi$ ambiguity is corrected by adding or subtracting $2 \pi$ to the phase of one of the data points so the phase difference is less than $\pi$. This process needs to be repeated for all data points until the phase difference between all adjacent data points is less than $\pi$.

Illustrating several important aspects of phase unwrapping in one dimension it is possible to understand some of the difficulties encountered in two-dimensional. The effects of errors in the unwrapping leads to streaks in the data where the unwrapping is incorrect.

\section{3}

\section{Deformation information extraction}

As it was discussed on the previous section, one way to obtain the deformation information from the analyzed object is to process the phase by applying the Hilbert Transform. In this section, we present the method used to extract and reconstruct the information from the phase that were more suited for the intended application. 
Chapter 2. Quantitative measurement of mechanical micrometer and sub micrometer deformations

\subsection{1}

Two measurements $\left(I_{a}\right.$ and $\left.I_{b}\right)$

As detailed throughout the chapter, the interferogram contains the phase information where we will be able to extract and reconstruct the deformation. Figure 2.9 shows the block diagram of the chosen method for the envisioned application.

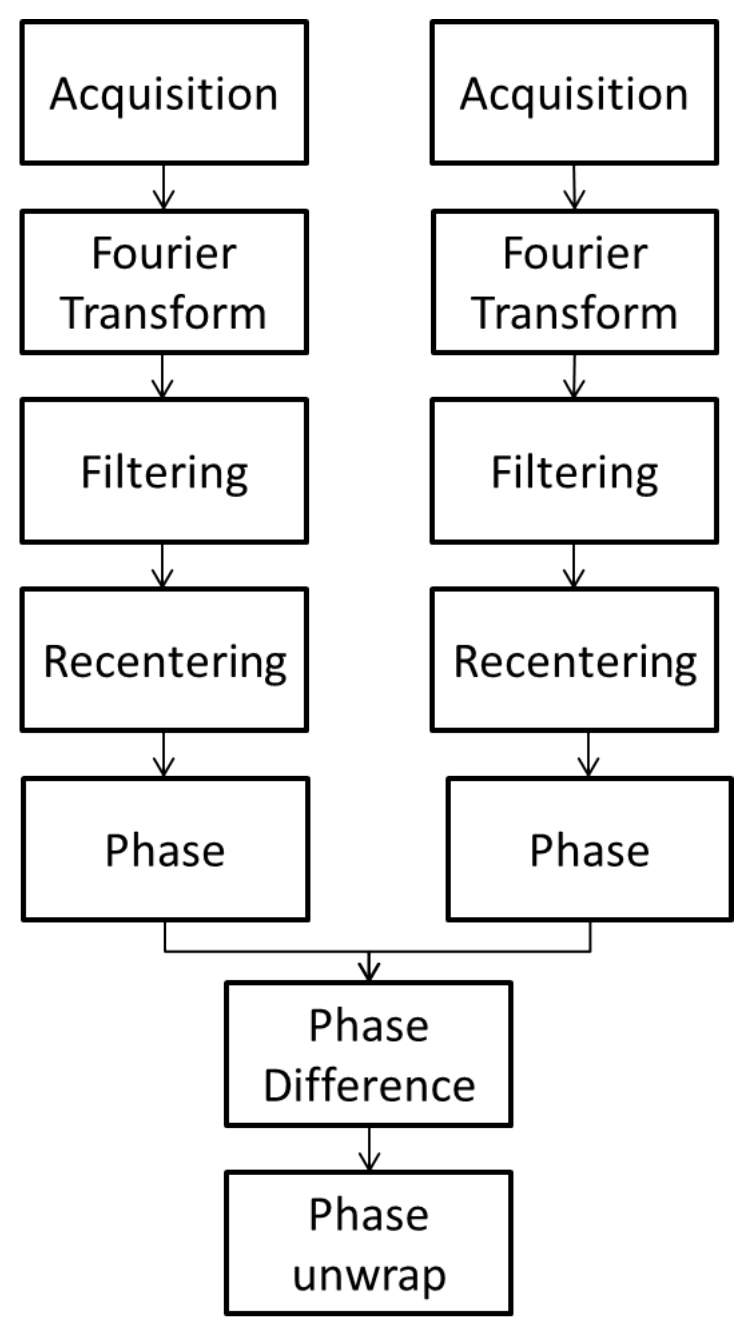

Figure 2.9: Block diagram for the deformation extraction from the phase using two interferograms.

We start by acquiring two interferograms, one before and another after the deformation is applied to the object, referred as reference and deformed, respectively. Concerning the deformation, it is important to note that the speckle pattern needs to maintain its correlation so when the differentiation is applied, the comparison is always related to the same speckle, before and after deformation. The phase information is coded in the speckle pattern and consequently, it is necessary to compare two images of the speckle. Figure 2.10 
Chapter 2. Quantitative measurement of mechanical micrometer and sub micrometer deformations

shows an example of an interferogram of a scattering target interfering with a reference plane wave.

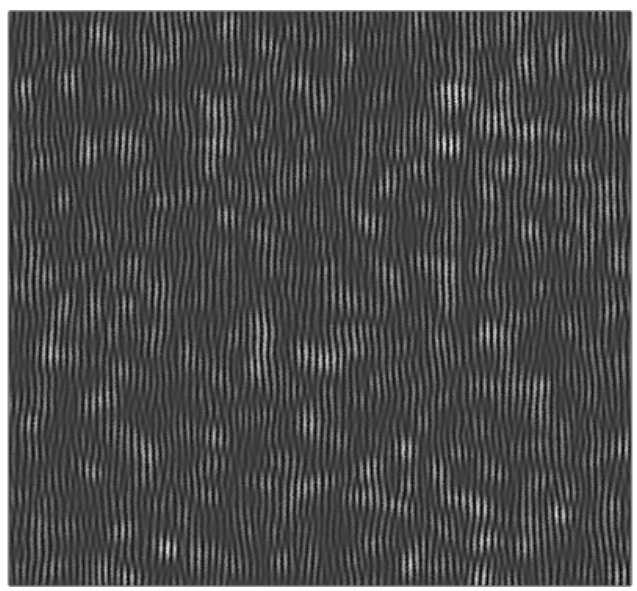

Figure 2.10: Example of an reference interferogram.

After recording the two interferograms, the Fourier Transform is applied on both. Since the spatial carrier method is used, i.e. a tilt was added to the system, the orders of the Fourier Transform are separated, as it was shown in Figure 2.6. Since the orders are available separately, the filtering process can be applied, selecting only the +1 order and then recentering it, as it is depicted in Figure 2.11.
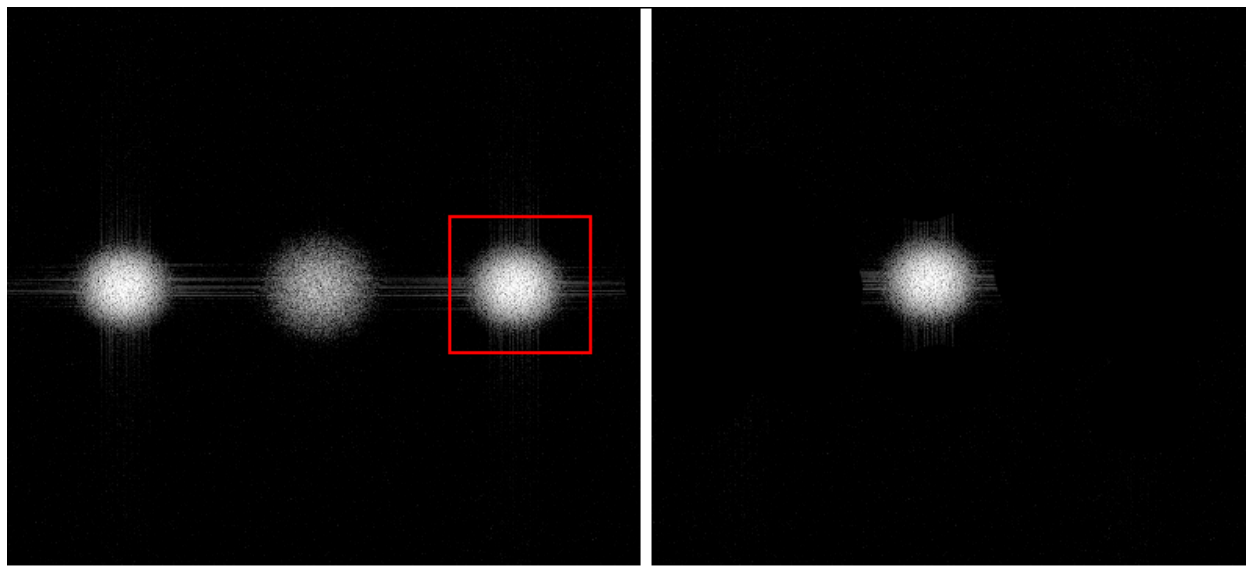

Figure 2.11: Fourier Transform of an interferogram with the +1 order selected (on the left) and subsequently filtered and recentered (on the right.

With the filtered result, the inverse Fourier Transform is applied to return to the time domain for both the reference and the deformed interferogram. Then, we access the phase as indicated by Figure 2.12, both depicted in Equation 2-22.

As indicated in Figure 2.9, the next step after acquiring the phase maps is to subtract the reference phase from the deformed phase to achieve 
Chapter 2. Quantitative measurement of mechanical micrometer and sub micrometer deformations
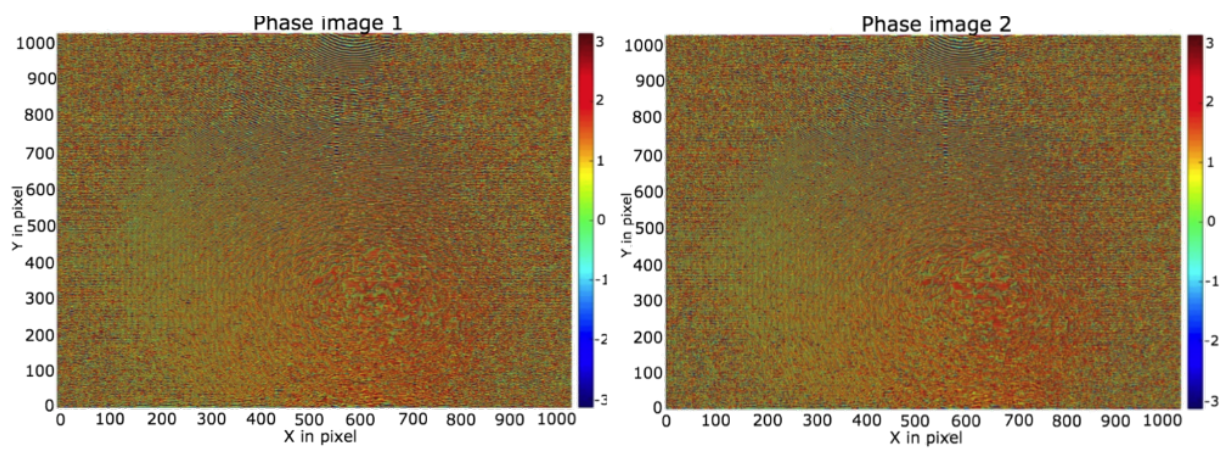

Figure 2.12: Example phase map of the interferogram of a scattering target a) before (reference) and b) after being deformed.

the deformation information. Figure 2.13 presents the results of the phase difference of the resulting phases shown in Figure 2.12. As explained in subsection 2.2.3, we need to eliminate the discontinuities from the resulting phase map by means of the phase unwrap. The result is a phase map of the deformation.

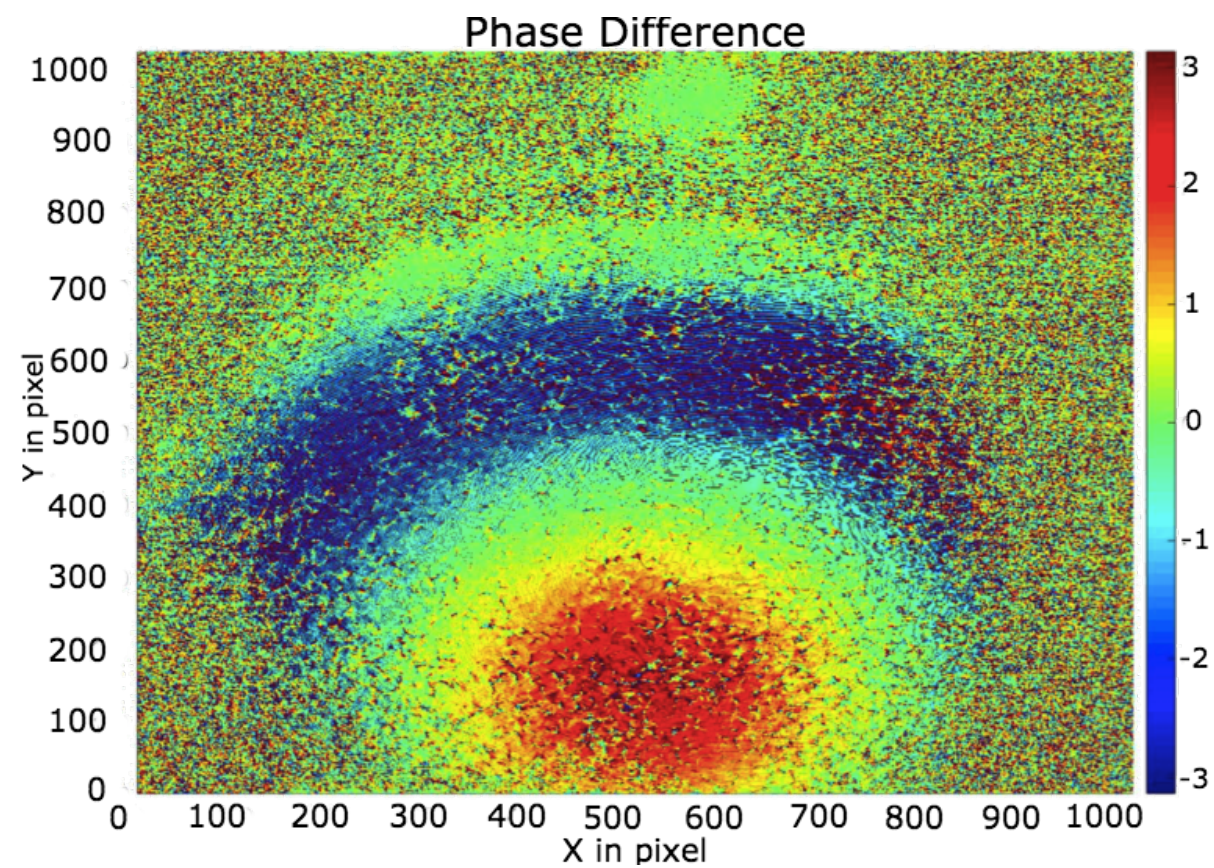

Figure 2.13: Phase difference between the reference and deformed phase maps presented in Figure 2.12. 


\section{3}

\section{Optical Methods for Vibrometry}

The goal of this work is to detect photoacoustic signals. To this end, different systems were set up and analyzed to determine the necessary parameters for the detection and acquisition of mechanical and ultrasound vibrations.

In the the following chapter we will introduce methods for vibrometry that are relevant to this thesis and that were experimentally set up. Among the systems analyzed are the Laser Optical Feedback Imaging (LOFI) and the stroboscobic interferometry, specifically using a Mach-Zehnder interferometry technique, both showing promising results. Their results will be presented and discussed in chapter 4 and a comparative study is analyzed in chapter 5 . We will also refer briefly to other relevant systems, as shearography and off-axis holography, and describe them in short detail necessary.

\section{1}

\section{Laser Optical Feedback Imaging}

The Laser Optical Feedback Imaging (LOFI) is an heterodyne interferometric imaging technique that profits from class B laser dynamics (microlasers, diode lasers, etc), and more precisely, its great sensitivity to the reinjection phenomenon (27). This allows for high precision measurements of the backscattered signal from a sample and to assess its reflectivity and phase with a spatial resolution of the order of $\mu m$ (28).

Since it is an interferometric technique, the equations presented on chapter 2 also apply for the LOFI, using space ( $\mathrm{x}$ ) instead of time (t). The orders $-1,0,+1$ of the Fourier Transform previously discussed will now be represented, respectively, by the peaks of the Bessel Function $J_{-1}, J_{0}, J_{1}$ in the spectral domain.

\section{Principle}

In LOFI, the heterodyne interference happens inside the laser cavity, between the intra-cavity light and the backscattered light from the imaged object. The backscattered light is frequency shifted as to create an intracavity optical beat that can be increased by the laser dynamics. In fact, if the 
frequency shift (the beat frequency) is close or equal to the laser's relaxation frequency, we amplify the optical beat by the order of $10^{6}$ with a $N d^{3+}: Y A G$ micro-laser $(29,30)$. Thus, the laser plays the part of both source and detector of photons, meaning it is an auto aligned system by principle (31).

This system is capable of obtaining amplitude and phase images of diffusing objects in non cooperative conditions, such as diffusing medium or weak reflective samples, thanks to signal to noise ratio of measurements being limited by the quantum noise of the laser. It is also capable of acquiring vibration frequencies, which is the main goal of this work. The LOFI method presents the following characteristics:

- Simplicity: Since the laser plays the part of the emitter and the receptor of photons, its set up is auto aligned by principle. This makes the LOFI system robust and easy to implement, an interesting characteristic when it comes to couple the system with other complex optical systems.

- Sensitivity: This double role also allows to overcome the noise of the photodiode because of the signal amplification phenomenon due to resonance. The SNR of the measurements is limited by the quantum noise of the laser. This allows to acquire images in difficult conditions, for example diffusing medium, with a low power source laser.

o Coherent detection: This characteristic allows the system to detect the ballistic photons and, therefore, acquiring images with high spatial resolution.

- Acquisition time: Since the detection is point wise, the amount of acquisitions is the same as the amount of pixels in the image. Also, each measurement needs to respect the response time of the laser (a few microseconds). Typically, for a 100x100 pixels image has an acquisition time of the order of a second.

- Depth of field: The coupling between the backscattered light and laser cavity mode act as a spatial filter comparable to a confocal system. This means a low field depth, allowing the optical sectioning of the sample.

Focusing on the ability of acquiring high spatial resolution images, the LOFI presents some interesting characteristics:

○ Images through diffusing mediums;

- Phase measurement of distant or low reflecting objects;

- Vibration measurements of distant or low reflecting objects. 


\section{Set up}

The LOFI system was initially used for image acquisition of the amplitude and the phase. It was later adapted for the vibration measurements. Figure 3.1 presents an experimental setup of the LOFI system, which is similar for both acquisitions mentioned, with minor changes to the detection system. The laser source is a $N d^{3+}: Y A G$ microlaser emitting a quasi Gaussian beam (mode $T E M_{00}$ ) at a wavelength of $\lambda=1064 \mathrm{~nm}$ and output power of order of $10 \mathrm{~mW}$ that is optically pumped by a laser diode at $808 \mathrm{~nm}$ (not represented in the Figure 3.1). The laser beam waist $W_{0}$ is around $50 \mu \mathrm{m}$. The detection system consists of a photodiode and a lock-in amplifier connected to the data acquisition card (DAC), as to process the signal and have access to the amplitude and the phase of the heterodyne modulation. For the vibration measurements, the lock-in amplifier acts as a filter and an oscilloscope that has the ability of registering the signal for further processing.

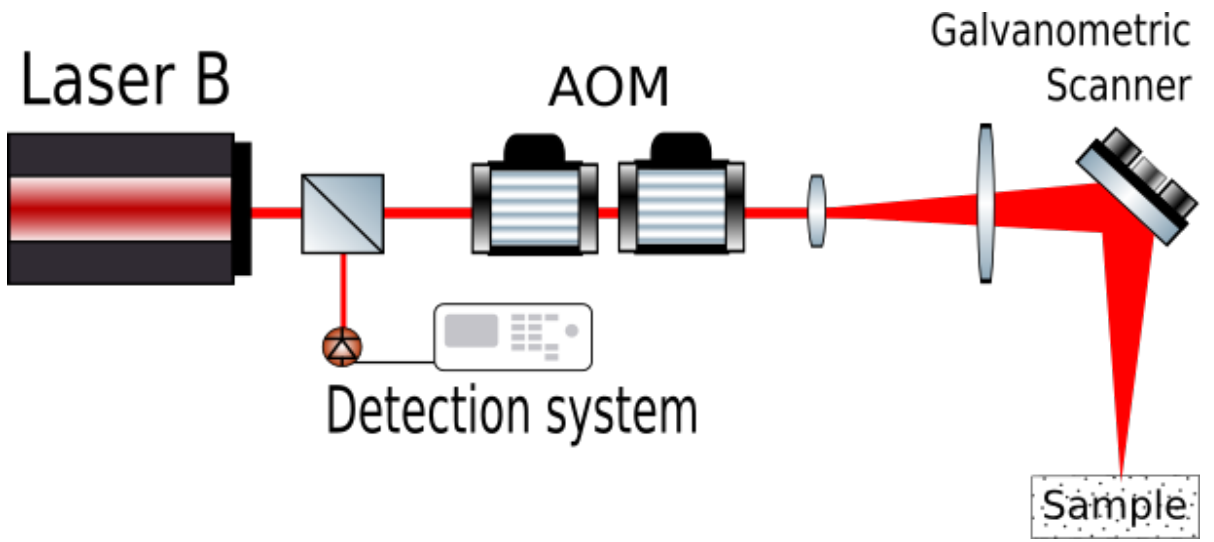

Figure 3.1: LOFI experimental setup.

Figure 3.2 shows an example of a typical output intensity of a $\mathrm{Nd}^{3+}$ : $Y A G$ microlaser measured with a photodiode over a period of time and it can be observed that it is a source of considerable intensity fluctuations, being able to reach a few percentage. Regarding the window marked in the plot, it is possible to perceive that the intensity fluctuates in a almost sinusoidal manner at the relaxation frequency $F_{R}$ of the laser.

The second plot of Figure 3.2 presents the laser's noise spectrum (square modulus of the Fourier transform of the laser's intensity signal). It can be noted that, for this type of laser, that the relaxation angular frequency $\Omega_{R}$ associated with the relaxation frequency $F_{R}$ is given by (32):

$$
\Omega_{R}=\sqrt{\gamma_{1} \gamma_{c}(\eta-1)}
$$



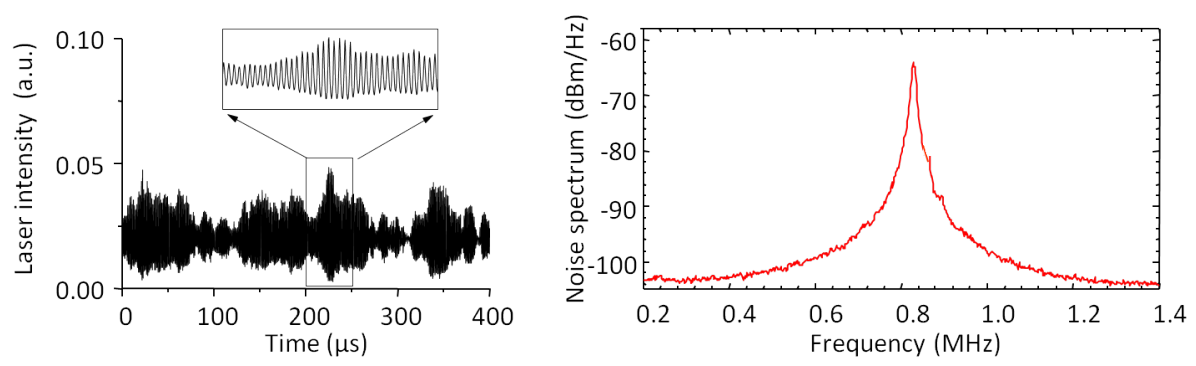

Figure 3.2: $\mathrm{Nd}^{3+}: Y A G$ microlaser intensity in function of time and its noise spectrum.

where $\gamma_{1}$ is the population inversion decay rate, $\gamma_{c}$ is the cavity decay rate and $\eta=\frac{B N_{0}}{\gamma_{c}}$ is the normalized pumping parameter. The width of the resonance curve is given by (32):

$$
\Delta \Omega_{R}=\eta \gamma_{1}
$$

The optical reinjection is characterized by three parameters: the shift frequency $F_{0}$, the distance laser-sample and the effective reflectivity of the sample $R_{e}$, that can be defined by the amount of light reinjected and detected by the laser (33). The frequency shift is generated by two acousto-optic modulators (AOM) that work on the +1 and -1 orders, respectively. The set of AOMs work as phase modulators. A shift of order of megahertz is obtained by subtraction, as depicted in Figure 3.3.

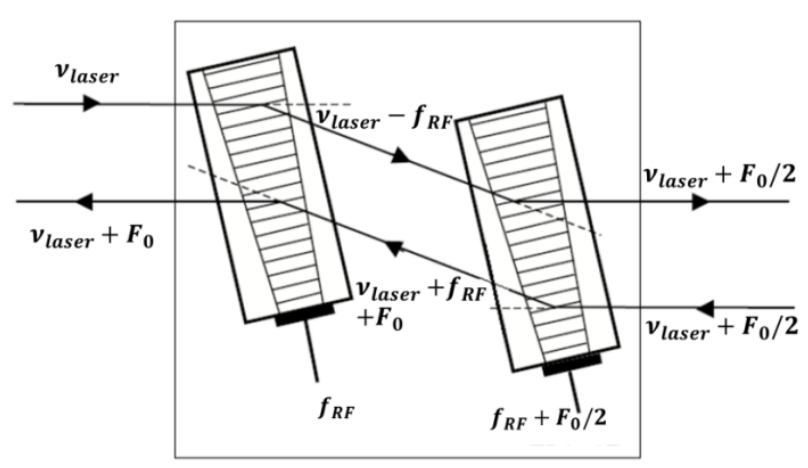

Figure 3.3: Frequency shift obtained by two acousto-optic modulators (AOM). $v_{\text {laser }}$ is the laser frequency before the shift. After a round trip, the light is frequency shifted of $F_{0}$.

The first AOM works at frequency $f_{R F}$ and diffracts the laser beam to -1 order. The laser beam frequency is shifted of $-f_{R F}$ and then it is sent to the second AOM, working at frequency $f_{R F}+F_{0} / 2$ and which diffracts the beam to +1 order. Thus, the laser beam is frequency shifted by $f_{R F}+F_{0} / 2$ and, after passing through both $\mathrm{AOM}$, the resulting frequency shift is of $F_{0} / 2$. Considering the round trip going through the AOMs, the beam is finally shifted 
by $F_{0}$. The backscattered light from the object makes a second passage by the $\mathrm{AOM}$ and is injected into the laser cavity, now optically shifted with frequency $F_{0}$. The frequency $F_{0}$ is chosen close to the relaxation frequency $F_{R}$ of the laser.

The laser beam must be focused on the object sampled to acquire the required measurements. The focusing optics is chosen according to the spatial resolution, the working distance and the angular field desired. The images are recorded point by point displacing the laser beam that illuminates the sample and each zone illuminated by the laser, on the surroundings of the focus point, correspond to a pixel of the image. Therefore, it is necessary to scan the laser beam onto the surface of the object sampled as to construct a complete image. This is achieved with the galvanometer scanner (i.e. a system of rotating mirrors run by a galvanometer motor), allowing the displacement of the laser beam without having to move the sample or the laser. It also limits the mechanical vibrations that strongly disturb the phase measurements.

Inside the laser cavity, an optical beat is produced between the intracavity electrical field and the reinjected electrical field with the shift frequency $F_{0}$, which has the effect of modulating the net gain of the laser, and hence the output intensity of the laser that will be measured. One part of the laser beam is thereby diverted to the detection system by a beam splitter with really low reflectivity in order to get the best signal to noise ratio $(\mathrm{SNR})(31,34)$.

The first application of the LOFI system was the acquistion of amplitude and phase information from the signal. An amplitude acquisition has information on the reflective contrast of the sample, illustrate by Figure 3.4, and a phase image has information about the surface profile or its vibrations modes if the object is moving, with an example depicted in Figure 3.5 (35). The images of vibration measurements with the LOFI system, which is the scope of the work, are presented in section 4.1 .

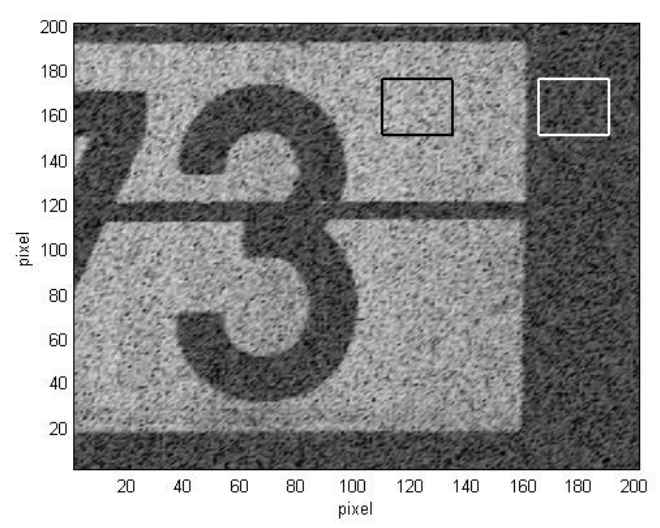

Figure 3.4: Example of an amplitude image of a license plate recorded by the LOFI. 
Figure 3.4 shows part of a license plate imaged through a diffusing medium by the LOFI system. The diffusing medium is diluted milk at $1.25 \%$ with $5 \mathrm{~cm}$ thickness. The losses by diffusion in the round trip are of the order of $30 \mathrm{~dB}$. The laser power is a few milliwatts at $1064 \mathrm{~nm}$ wavelength. The laser beam waist is $W_{0}=230 \mu \mathrm{m}$ at the sample. The license plate is located at a distance $l=3 m$ of the scanning mirror. The images are $200 \times 200 \times 200$ pixels with a field of view of around $20 \mathrm{~cm}$ and a exposure time of $100 \mu \mathrm{s}$ per pixel.

The numbers on the license plate are observable despite the $30 d B$ loss and the low laser power. Two parts can be distinguished in the image. On the left with the analyzed sample and on the right without it. This allows us to calculate the mean SNR over 625 pixels (small squares on Figure 3.4). The black band on the image corresponds to the quantum noise of the laser. To obtain it, the laser beam is temporarily masked between the beam splitter and the frequency shifter. So, the measured signal is the laser noise at frequency $F_{0}$ since there is no shifted light at this frequency reinjected in the laser. The difference between this band and the part of the image without the sample (square on the right on Figure 3.4) is what determines that the noise in this image is the quantum noise of the laser. Therefore, this image is limited by the photon noise.

When the refraction index and the thickness are known to the axial profile, the measurement of the phase $\Phi(x, y)$ and the relation $z(x, y)=\frac{\lambda}{2} \frac{\Phi(x, y)}{2 \pi}$ allows to obtain the profilometry (33). The axial resolution depends on the mechanical stability and it is of the order of $10 \mathrm{~nm}$. Figure 3.5 shows the profile of a resolution test target, plots of silicon with $200 \mathrm{~nm}$ height and width of $6 \mu m$.

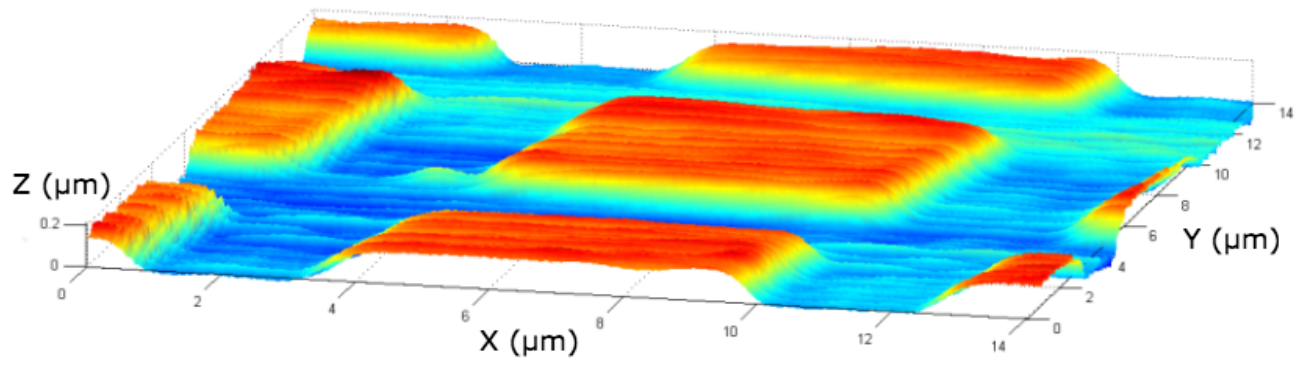

Figure 3.5: Example of an phase profile of a silicon resolution target recorded by the LOFI.

\section{Signal}

The LOFI system can be studied from the rate equations for an injection laser with a frequency shifted optical injection $(32,29,30)$. Considering the 
dynamic behavior of a laser submitted to a low optical injection $\left(R_{e} \ll 1\right)$ frequency shifted, the modified rate equations can be described as:

$$
\begin{gathered}
\frac{d N}{d t}=\gamma_{1}\left(N_{0}-N\right)-B N I \\
\frac{d I}{d t}=\left[B N-\gamma_{c}+2 \gamma_{c} \kappa_{o p t} \sqrt{R_{e}} \cos \left(\Omega_{0} t-\omega_{c} \tau\right)\right] I
\end{gathered}
$$

where $\mathrm{N}$ is the population inversion, $\gamma_{1} N_{0}$ is the pumping rate, $\mathrm{B}$ is related to the Einstein coefficient, I is the number of photons in the laser cavity, $\kappa_{\text {opt }}$ is the ratio between the optical output power and the optical power that arrives at the target, $\omega_{c}$ is the angular frequency of the laser cavity, $\Omega_{0}=2 \pi F_{0}$ is the shift pulsation and $\tau$ is the traveling time between the laser and the sample. $R_{e}$ is the effective reflectivity coefficient of the sample, taking into account the diffusing aspect of the object imaged as well as the recovery between the wave emitted by the laser and the wave injected into the laser.

Solving the equation system (3-3) and (3-4) allows to determine the heterodyne modulation that comes out from the laser, i.e. the LOFI signal $S_{L O F I}$. In fact, after the analysis of small perturbations and considering only $\Delta N$ and $\Delta I$, it is possible to demonstrate $(29,30)$ that, on the laser output, there is a relative variation $\Delta P_{\text {out }}$ of the laser power in the form of:

$$
\frac{\Delta P_{\text {out }}}{\left\langle P_{\text {out }}\right\rangle}=S_{L O F I}=2 G_{L O F I}\left(\Omega_{0}\right) \kappa_{\text {opt }} \sqrt{R_{e}} \cos \left(\Omega_{0} t-\omega_{c} \tau+\phi\left(\Omega_{0}\right)\right)
$$

where $\left\langle P_{\text {out }}\right\rangle=\gamma_{c} I$ represents the average number of photons emitted per second by the laser. Therefore, $G_{L O F I}\left(\Omega_{0}\right)$ and $\phi\left(\Omega_{0}\right)$ can be expressed as:

$$
\begin{gathered}
G_{L O F I}\left(\Omega_{0}\right)=\frac{\gamma_{c} \sqrt{\left(\Delta \Omega_{R}\right)^{2}+\Omega_{0}^{2}}}{\sqrt{\left(\Omega_{R}^{2}-\Omega_{0}^{2}\right)^{2}+\left(\Delta \Omega_{R}\right)^{2} \Omega_{0}^{2}}} \\
\phi\left(\Omega_{0}\right)=\arctan \left[\frac{\Omega_{0}\left[\left(\Omega_{R}^{2}-\Omega_{0}^{2}\right)+\left(\eta \gamma_{1}\right)^{2}\right]}{\eta \gamma_{1} \Omega_{R}^{2}}\right]
\end{gathered}
$$

The heterodyne beat at the $\Omega_{0}$ pulsation is amplified by the factor $G_{L O F I}$. The form of this amplification factor can be observed on Figure 3.2 since the fluctuations of the laser intensity comes from the resonant amplification of the quantum noise of the laser.

The equation (3-5) shows that the LOFI signal is the heterodyne beat amplified by the factor $G_{L O F I}$. Therefore, its amplitude is proportional to the sample's effective reflectivity in amplitude and the phase is proportional to $\tau$, the traveling time between the distance $d$ laser-sample. In a medium 
with a constant refractive index $n, \tau=2 n d / c$, where $\mathrm{c}$ is the speed of light. This amplification $G_{L O F I}$ can be adjusted according to $\Omega_{0}$ and at resonance $\left(\Omega_{0}=\Omega_{R}\right)$ with $\Omega_{R} \gg \gamma_{1}$ (for class B lasers), equation (3-5) becomes:

$$
\frac{\Delta P_{\text {out }}}{\left\langle P_{\text {out }}\right\rangle}=\frac{2 \gamma_{c}}{\eta \gamma_{1}} \kappa_{\text {opt }} \sqrt{R_{e}} \cos \left(\Omega_{0} t-\omega_{c} \tau+\phi\left(\Omega_{0}\right)\right) .
$$

The amplification factor is proportional to the ratio between the cavity decay rate and the population inversion decay rate. Table 3.1 lists this ratios for different types of class B lasers. It can be observed that, for the microlasers, the ratio can reach 1 million, explaining the choice for this particular laser on the current LOFI set-up. In these conditions, a reflectivity of $10^{-12}$ was measured with a 100\% contrast (30). The LOFI sensor's response time is directly connected to the population inversion decay rate $\gamma_{1}$, so a higher response time translates into a higher gain $G_{L O F I}$ and the microlaser is the better compromise between the two required parameters.

Table 3.1: LOFI Amplification for different class B lasers.

\begin{tabular}{lcccc}
\hline Laser & $\lambda$ & $\gamma_{1}$ & $\gamma_{c}$ & $\gamma_{c} / \gamma_{1}$ \\
\hline \hline Titanium sapphire & $0,7-1,1 \mu \mathrm{m}$ & $3,1 \cdot 10^{5} \mathrm{~s}^{-1}$ & $1,9 \cdot 10^{6} \mathrm{~s}^{-1}$ & 6 \\
Semiconductor & $0,8-4 \mu \mathrm{m}$ & $1 \cdot 10^{9} \mathrm{~s}^{-1}$ & $1 \cdot 10^{12} \mathrm{~s}^{-1}$ & $1 \cdot 10^{3}$ \\
Nd:YAG & $1064 \mu \mathrm{m}$ & $4,3 \cdot 10^{3} \mathrm{~s}^{-1}$ & $6,3 \cdot 10^{7} \mathrm{~s}^{-1}$ & $1,5 \cdot 10^{4}$ \\
Ruby & $694,3 \mu \mathrm{m}$ & $333 \mathrm{~s}^{-1}$ & $1 \cdot 10^{8} \mathrm{~s}^{-1}$ & $3 \cdot 10^{5}$ \\
Microlaser & $1064 \mu \mathrm{m}$ & $4,3 \cdot 10^{3} \mathrm{~s}^{-1}$ & $5 \cdot 10^{9} \mathrm{~s}^{-1}$ & $1,2 \cdot 10^{6}$ \\
\hline
\end{tabular}

\section{SNR}

As shown in Figure 3.4, the LOFI gain allows, under certain conditions, to overcome the detection noise and to be limited by the photon noise. There are two main origins for the LOFI signal's noise:

o Detection noise: it is essentially the thermal noise of the photodiode, the lock-in amplifier or the DAC. This is a white noise and hence independent of frequency (dotted curve of Figure 3.6)(36).

- Photon noise: this is not a white noise anymore, due to the effects of the laser dynamics that modifies the form of the noise spectrum (cyan line in Figure 3.6).

Figure 3.6 shows the spectrum of the injected laser's intensity with a shift frequency of $F_{0}=0,82 \mathrm{MHz}$. So, the peak at $0,82 \mathrm{MHz}$ frequency corresponds to the LOFI signal. As indicated by the blue curve in Figure 3.6, the SNR is equal to the height of this peak. The LOFI technique is limited only 


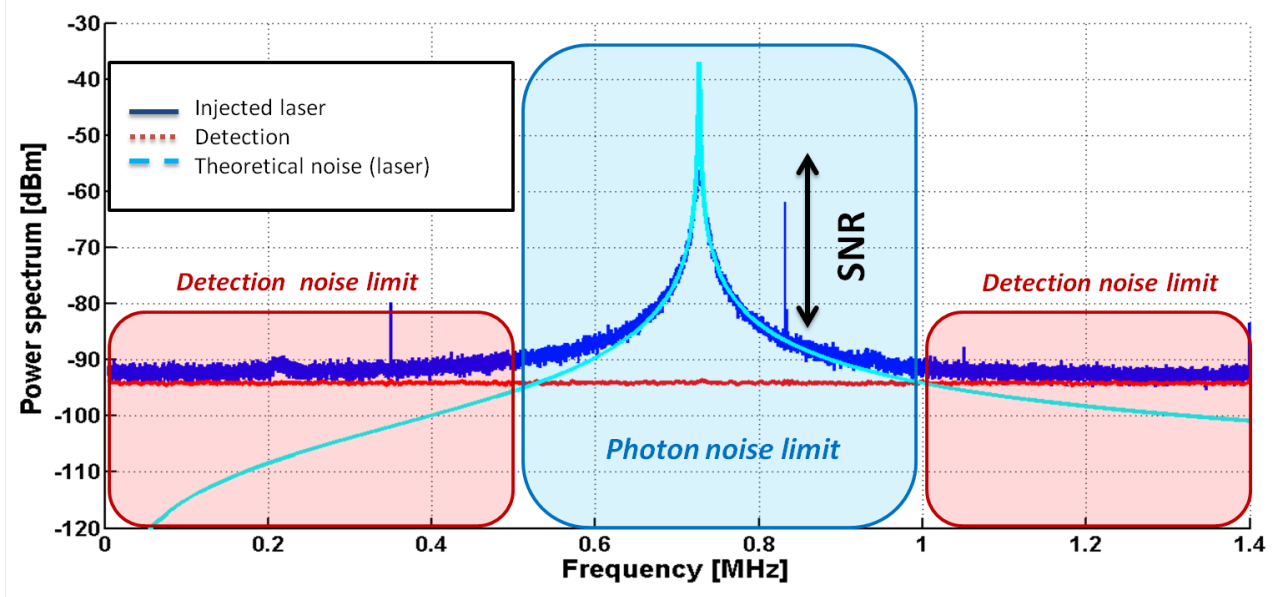

Figure 3.6: Power spectrum measure from a pumped laser (laser and detection) and the laser theoretical noise.

by the photon noise when the laser's quantum noise is superior to the detection noise (dotted red line), corresponding to a very specific band of frequency shift $F_{0}$ ( the $[0,5-1] \mathrm{MHz}$ region of the plot).

This frequency region stands for the frequencies for which the spectrum of the theoretical noise is superior to the detection noise, and outside it the LOFI technique will be limited by the detection noise (34). Within this frequency band limited by the photon noise, the SNR is independent of the frequency shift $F_{0}$ and it is only limited by the number of photons injected by the target into the laser, and thus the expression for the SNR is (31):

$$
S N R=\sqrt{T_{\text {int }} R_{e}\left\langle P_{\text {out }}\right\rangle} \kappa_{\text {opt }},
$$

where $T_{\text {int }}$ is the exposure time of the measurement. A $S N R=1$ corresponds to a photon injection during the measure time $T_{\text {int }}$.

The LOFI signal (Equation 3-8) contains information about the amplitude that is proportional to the effective reflectivity of the sample but it also contains information about the phase that is proportional to the traveling time between laser and imaged object. Scanning the laser beam onto the target, it is possible to do a mapping of both parameters and acquire $R_{e}(x, y)$ and $\Phi(x, y)$ with $x$ and $y$ being the cross plan in the direction of the laser beam. The gain $G_{L O F I}$ allows the system to register these mappings in non cooperative conditions like imaging through diffusing medium.

Varying the distance between laser and sample of $\lambda / 2$ translates into a phase variation of $2 \pi$ while a variation superior to $\lambda / 2$ implies a measured phase ranging between the $[-\pi,+\pi]$ interval. In this latter case, it is necessary to unwrap the phase, as it was discussed in subsection 2.2.3. Measuring $\Phi(x, y)$ dynamically enables to determine the position of the sample over time and 
perform vibrometry. Therefore, knowing $\Phi(x, y, t)$ allows the determination of the amplitude and the spectrum of a mechanical vibration. In high frequency vibrometry, the LOFI technique is used for 2D dynamic mapping of ultrasound vibrations of frequencies ranging from a few kilohertz to a few megahertz. In the case of a sample moving at a frequency higher than some $\mathrm{kHz}$, the lock-in amplifier is no longer suitable because of the limited bandwidth. Therefore, the phase $\Phi(x, y, t)$ can be determined by the acquisition of the temporal trace of the LOFI signal $\left(S_{L O F I}\right)$ and calculating its Hilbert Transform (37).

$$
\Phi(x, y, t)=\arctan \left(\frac{H\left(S_{L O F I}(x, y, t)\right)}{S_{L O F I}(x, y, t)}\right)-2 \pi F_{0} t
$$

with $H\left(S_{L O F I}(x, y, t)\right)$ being the Hilbert Transform of the LOFI signal.

This processing allows to obtain the vibration function of the sample and know its amplitude and phase over time. Each temporal trace comprises one of the pixels from one vibration's video. The results for high frequency vibrometry using the LOFI system will be presented in section 4.1. One advantage is that the LOFI is a resonant system, amplifying the interference signal that comes from the noise.

\section{2 \\ Shearography}

Shearography (38), a technique born from speckle shearing interferometry $(39,40)$, became a important tool for diagnostic purposes, allowing direct displacement gradients measuments. It is a laser based method that offers the advantages of interferometric non contact full-field sensitivity due to an optical configuration that is more resilient to environmental disturbances and vibrations than other techniques (41). The most significant advantage in shearography is its capability of measuring the derivatives of displacements with respect to the shear direction instead of displacements $(42,43)$.

The surface of the object is illuminated by a laser as to form a speckle pattern. The light reflected from the object is imaged by the shearing device, combining the speckle pattern with a laterally shifted version of itself and recorded using a CCD camera. The ray scattered from one point on the surface interferes with its neighbor, and when the object is deformed, a relative displacement occurs between the two points. This relative displacement produces a relative phase change between the rays. Two images are acquired, before and after a deformation is applied, for processing and analysis of the phase difference, which contains the information on the displacement derivative.

The system chosen for this work uses a Mach-Zehnder interferometer 
as the shearing device. This experimental set-up, first introduced by Pedrini (44), uses the spatial carrier method for the reconstruction of the optical phase variation due to the object deformation. As Figure 3.7 shows, a lens expands the laser beam to illuminate the object and it is imaged by a second lens, with the diaphragm in front of the camera to control the speckle size. The first beamsplitter separates the beam in two to follow different paths, reflecting on mirrors M1 and M2 and being recombined by the second beamsplitter to be imaged by the CCD camera. Mirror 2 (M2) adds the shear by being laterally translated while mirror 1 (M1) is tilted to set an angle between the two beam, thus producing a carrier frequency.

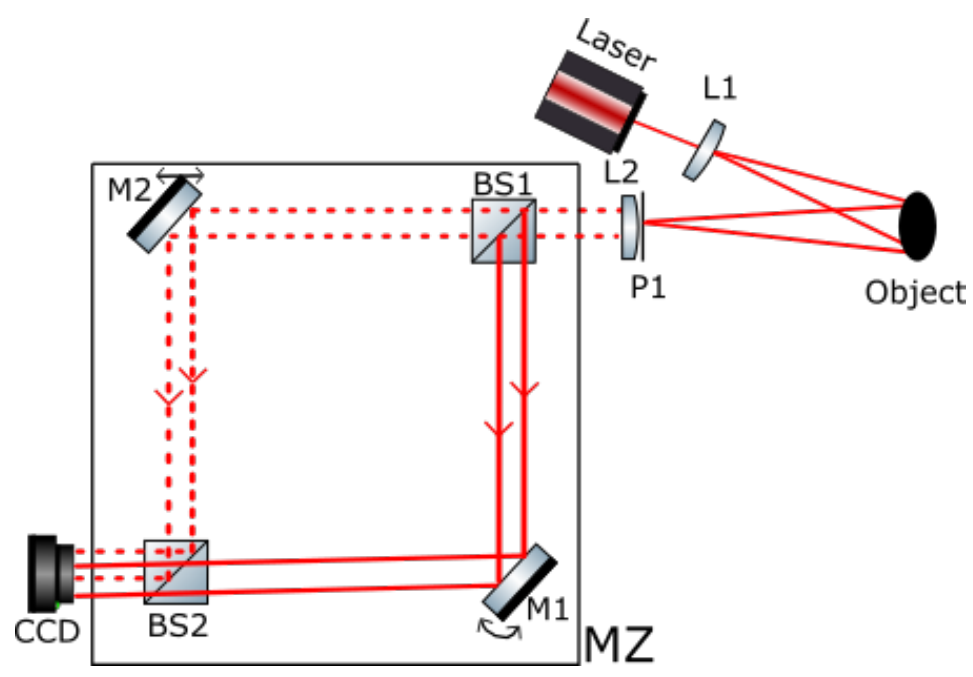

Figure 3.7: Shearography experimental set-up for the digital shearing based on a Mach-Zehnder interferometer. The mirrors are indicated by M1 and M2, P1 is a diaphragm, BS1 and BS2 are the beamsplitters, and L1 and L2 represent the lenses.

A He-Ne laser with $632.8 \mathrm{~nm}$ wavelength and output power of $15 \mathrm{~mW}$ is used as the light source, illuminating the diffusing object after being expanded by the lens L1 $(f=50 \mathrm{~mm})$. The light reflected from the the metallic plate enters through a second lens (L2 with $f=150 \mathrm{~mm}$ ) to the shearing device. The CCD camera used has a resolution of 2048 px X 1088 px, pixel size of 5.5 $\mu$ X $5.5 \mu$ with 12 bit depth and a frame rate of 165 fps.

As the reference signal, one image is acquired before any deformation is applied to the sample. A mechanical deformation is employed, and a second image is recorded by the CCD camera, and both data will be postprocessed and analyzed.

Another important part of the process is the determination of the shear magnitude, which is a significant source of uncertainty. The technique chosen for this project is the digital cross-correlation (45). Two images must be acquired, each one with a different arm of the interferometer blocked, and 
then the digital cross-correlation is calculated, obtaining from this the shift between the two images by the separation of the correlation peak from the center. The error uncertainty for our calculations is $\pm 0.00275 \mathrm{~mm}$. Therefore, the resulting shear in the Figure 3.8 example is, approximately, $\Delta x=-0.144$ $\mathrm{mm}$ and $\Delta y=0$, where the minus sign indicates the direction of the shear.

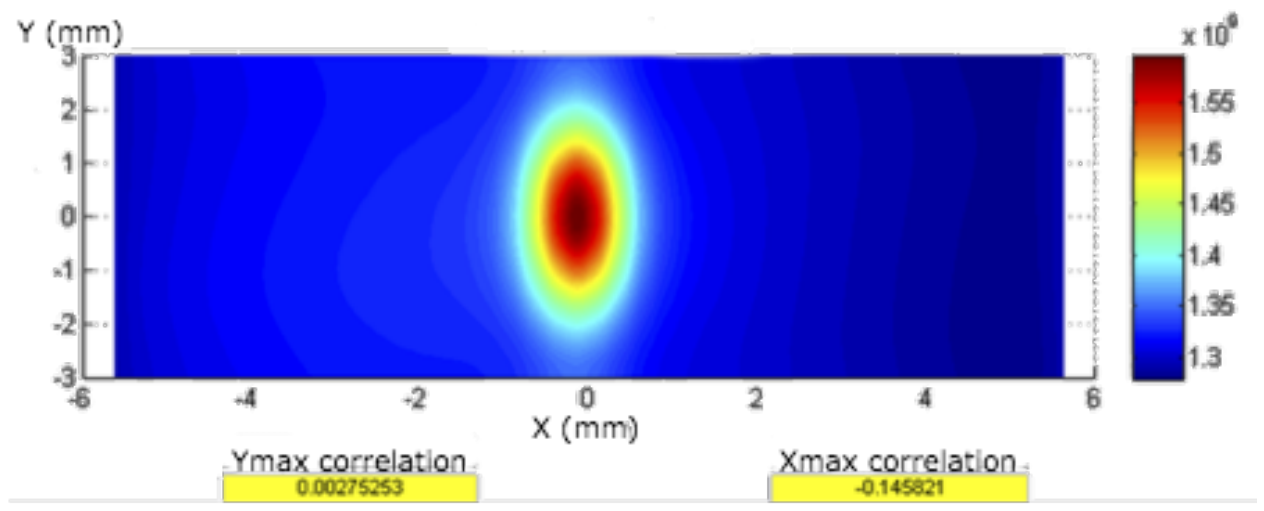

Figure 3.8: Result of a shear magnitude calculation using the digital crosscorrelation method.

\subsection{1}

\section{The Shearing Relation}

The shearing relation is related to changes to the optical path. The ray traveling from the light source through point $P(x, y, z)$ to the CCD sensor has a change to its optical path $\Delta l$. If we consider a neighboring point $P_{1}$ with a shear $\Delta x$ (and considering $\Delta y=0$ ) which is displaced to $P_{1}^{\prime}(x+\Delta x+u+\delta u, y+v+\delta v, z+w+\delta w)$, its path change $\Delta l^{\prime}$ is changed due to the deformation.

The displacement vector is considered as $(u+\delta u, v+\delta v, w+\delta w)$. Since the interference occurs between a point $\mathrm{P}$ and its neighbor $P_{1}$ laterally shifted by $\Delta x$, the relative path change due to deformation is:

$$
\Delta \phi=\frac{2 \pi}{\lambda}\left[\Delta l^{\prime}-\Delta l\right]=\frac{2 \pi}{\lambda}\left[A \frac{\delta u}{\delta x}+B \frac{\delta v}{\delta x}+C \frac{\delta w}{\delta x}\right] \delta x .
$$

$\mathrm{A}, \mathrm{B}$ and $\mathrm{C}$ are sensitivity factors dependent on the observation and illumination directions. Equation 3-11 shows that the relative phase change is a function of the relative displacement between points $\mathrm{P}$ and $P_{1}$. If we consider a small amount of shear, the shearing relation (46) for $\Delta x$, can be represented approximately as:

$$
\Delta \phi=\frac{2 \pi}{\lambda}\left[A \frac{\partial u}{\partial x}+B \frac{\partial v}{\partial x}+C \frac{\partial w}{\partial x}\right] \Delta x
$$

This shows that the phase difference depicts the derivatives of displacement 
with respect to the direction of shear. The same approximation can be extended for a shear in the y-direction.

The factors A and B represent the in-plane displacement derivatives while factor $\mathrm{C}$ is the out-of-plane displacement component. Assuming that, usually, the light source and the CCD are in the $x-z$ plane, and the viewing direction is along the $z$ axis. If we consider that the object size is small in relation to $Z_{o}$ and $R_{s}$ :

$$
\left.\begin{array}{r}
A=-\sin \theta \\
B \simeq 0 \\
C=-(1+\cos \theta)
\end{array}\right\},
$$

where $\theta$ is the angle between the illuminating and viewing directions. Therefore, Equation 3-12 becomes:

$$
\Delta \phi=-\frac{2 \pi}{\lambda}\left[(\sin \theta) \frac{\partial u}{\partial x}+(1+\cos \theta) \frac{\partial w}{\partial x}\right] \Delta x .
$$

If we illuminate the object with a small angle with the normal, i.e. $\theta \approx 0$ we minimize the in-plane displacement derivatives, therefore we can consider only the out-of-plane displacement component. Thus,

$$
\Delta \phi=\frac{4 \pi}{\lambda} \frac{\partial w}{\partial x} \Delta x
$$

With a single pair of speckle pattern images (before and after the loading) the phase map can be evaluated (47). The acquisition rate is only limited by the CCD sensor's capability. Since we acquire the derivatives of the displacement, a integration is needed to recover the deformation. For in-plane displacement and muti-component measurements, (48) offers a review of the available methods.

Despite some encouraging results, we concluded that digital shearing was not the best approach for the desired acquisition of acoustic waves, with major limitations. The information contained in the derivative of the deformation is not sufficient to access the quantitative measurements of a vibration acquired from a diffusing object and the measurement size of the system is relatively small. The variations of the deformation should be fast to be properly measured by the shearographic system, which is not the case for our tests. Also, the system presented a reduced stability, making it difficult to further adaptation into a photoacoustic system (49). 


\section{3}

\section{Off-axis holography}

While holography and shearography are generally treated as separate techniques, examination of the process of generating, recording, and subsequent reconstruction of information carrying optical interference patterns reveals that their underlying principles are basically similar (50).

Based on a Michelson interferometer, the mirror M2 is tilted to create the carrier frequency and, therefore, separate the orders on the spatial domain (51). Figure 3.9 presents the set up assembled for the experimental tests. At the exit of the interferometer a hologram is created by the interference between the object wave and the reference wave, at the CCD camera.

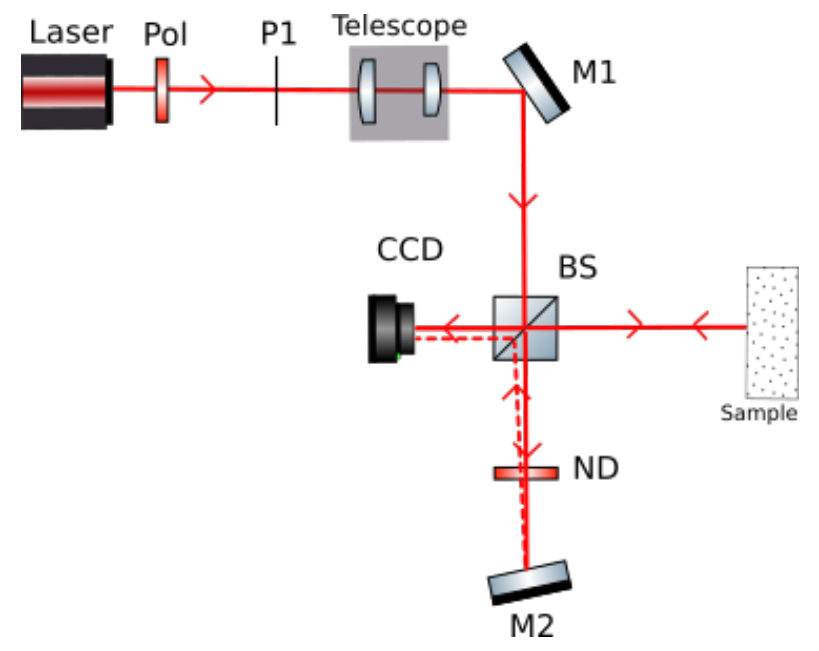

Figure 3.9: Off-axis holography experimental setup basically consists of a Michelson interferometer.

As for the Shearography set up, the off-axis holography also has a reference arm where an angle is added to the reference mirror (52). We ran a comparison test between both systems as to determine the comparability of the angle added to the mirror. Figure 3.10 shows the results of this comparison test between both systems for the acquired $\alpha$ angle. The angle was calculated for each system as follows:

$$
\begin{aligned}
\alpha_{m c} & =\frac{\lambda}{2(i / G)} ; \\
\alpha_{m z} & =\frac{\langle\Delta \Phi\rangle \lambda}{4 \pi \Delta x},
\end{aligned}
$$

where $\mathbf{i}$ is the number of fringes, $\mathbf{G}$ is the magnifying factor added by the lens, $\Delta x$ is the shear and $\langle\Delta \Phi\rangle$ is the mean value throughout the phase difference map. 


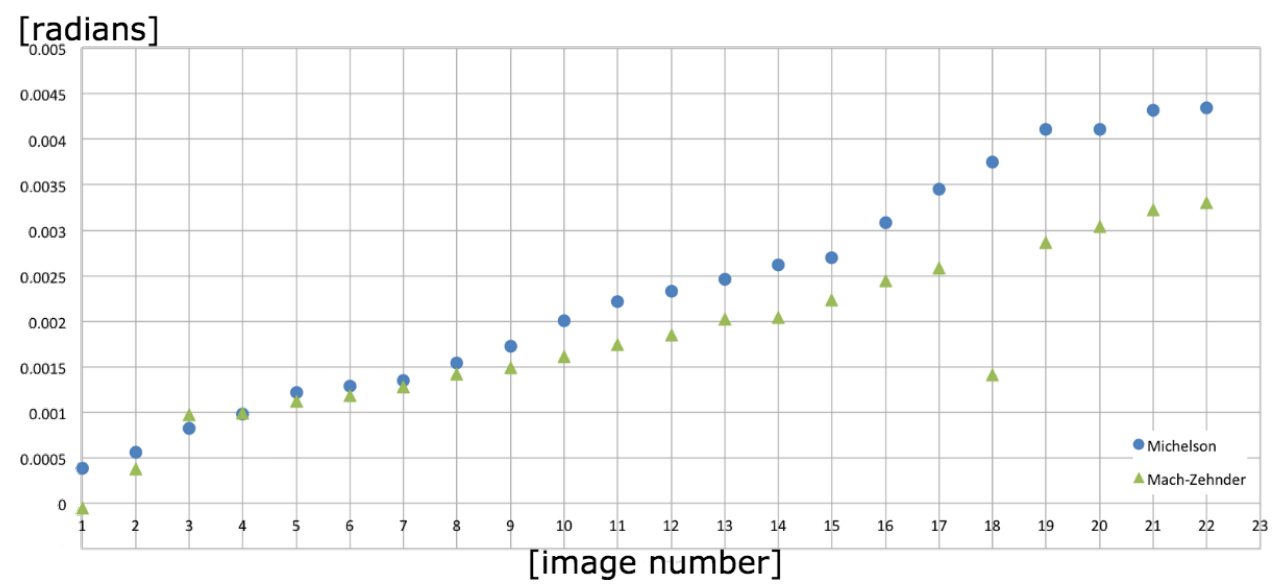

Figure 3.10: Plot of the angle measured in each image by both systems.

As the graph shows, apart from a few points, the measurements match for both systems and also with the angles set to the mirror. We repeated the same test (Figure 3.11), this time moving the angle from one side, passing through zero and continue to the other side.

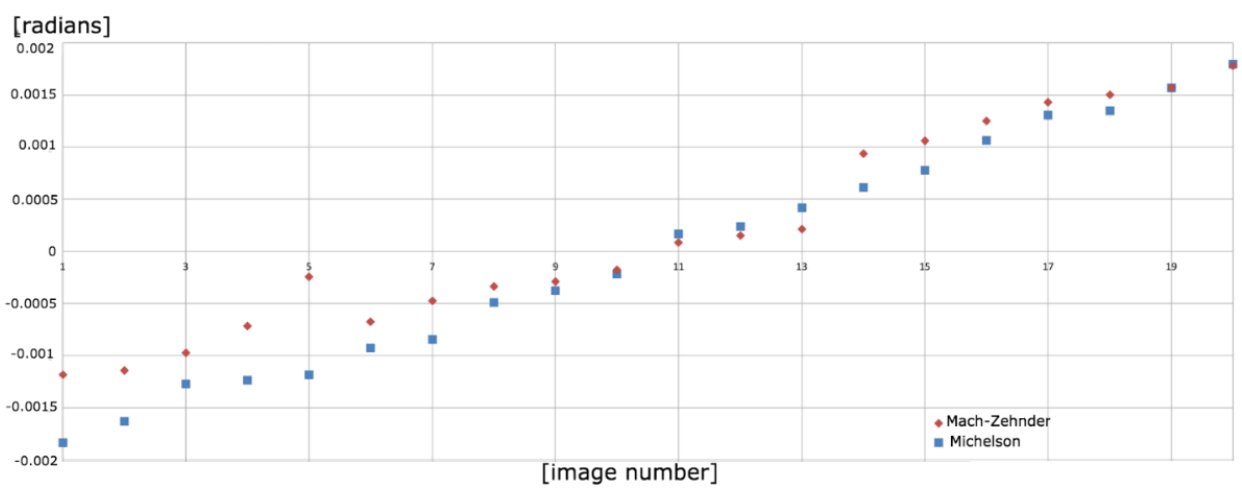

Figure 3.11: Plot of the repeated test for the angle measured in each image by both systems.

The results shown above presented promising factors to move forward with the assessment of this interferometric set up. However, some issues became apparent with the manipulation of the system.

One main issue found with this system regarding the intended application was its sensibility to external disturbances. Any contact with the optical table where the system was mounted would generate vibration waves on the image. Since the goal is to integrate this system in a photoacoustic imaging system, this would be impractical to detect the vibration and ultrasound signals intended. For this reason, we passed to the study of the Stroboscopic MachZenhder, presented in the next section. 


\section{4}

\section{Stroboscopic Mach-Zehnder}

Figure 3.12 presents the experimental setup for the stroboscopic MachZehnder (53). A He-Ne linearly polarized laser beam with wavelength $\lambda=$ $633 \mathrm{~nm}$ with output power of $15 \mathrm{~mW}$ and beam diameter of $0,70 \mathrm{~mm}$ passes by an AOM that is modulated at $40 \mathrm{kHz}$ by continually shifted pulses. The laser beam power exiting the $\mathrm{AOM}$ is $10 \mathrm{~mW}$ but the maximum output power arriving at the sample is $3,9 \mathrm{~mW}$, which is consistent with the limits established for biomedical applications (54).

Placing a diaphragm after the AOM, we are able to filter the zero order to work only with the modulated first order, running at $80 \%$ of the total output power. The linearly polarized beam coming out of the AOM is then shifted by the half-wave plate to arrive at the entrance of the Mach-Zehnder interferometer. The combination of the polarizing beam and the half-wave plate allows the balancing of the light intensity on each arm of the interferometer. This is required since both the reference arm and the object arm need to have the same intensity as to achieve optimum fringe pattern contrast, therefore, playing with the polarization achieves that goal without the necessity of adding more optical elements to the system.

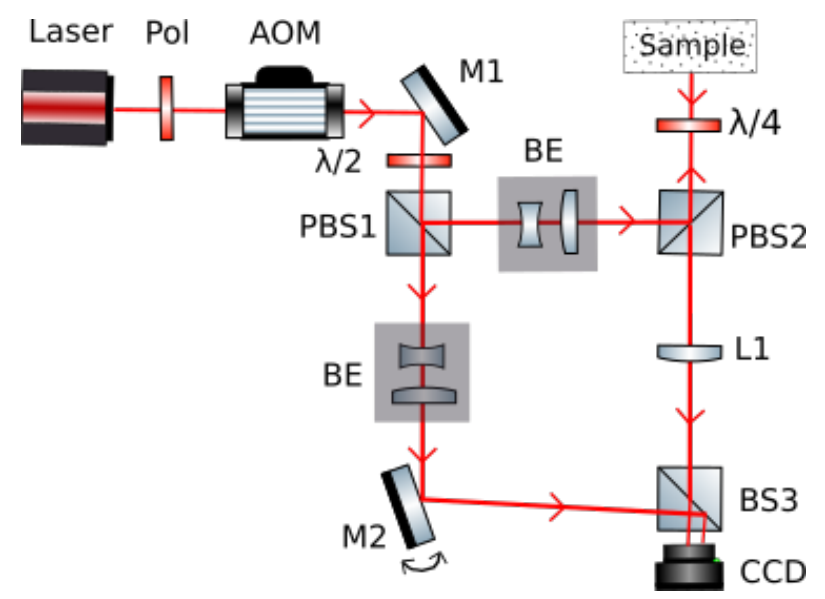

Figure 3.12: Stroboscopic Mach-Zehnder experimental setup.

After the polarizing beam splitter (PBS) separates the beam in two, one arm defined as reference, i.e. no modifications are made to the beam, and the object arm, where the beam will illuminate the evaluated sample. A beam expander is placed in both arms to increase the size of the beam, with the particular interest of illuminating a bigger area of the object's surface. In the reference arm, the beam if reflected by a mirror to be recombined with the beam backscattered from the sample on the detector, the CCD camera. This mirror is tilted with an angle $\alpha\left(\alpha<10^{\circ}\right)$ to create the spatial carrier frequency 
described in chapter 2. On the object arm, the beam exiting the beam expander passes through another PBS before illuminating the sample.

The light backscattered from the sample reenters the PBS and, before being recombined with the reference beam, goes through an imaging lens and a diaphragm. The diaphragm has the purpose of controlling the size of the speckle. With the lens placed to image the surface of the object in the CCD camera, acquiring the information without the need of the Fresnel approximation. It also adds a magnification factor, depending on the relation between the object/lens and lens/camera distances and the focal distance of the lens. In this system, we had a 1.5 magnification factor in the resulting image.

To characterize the set up in terms of its optical resolution and magnification factor, images of a resolution test target were acquired. For the characterization, the reference arm of the interferometer (reflected light from the M2 mirror on Figure 3.12) was blocked and only the signal from the object arm was captured by the CCD sensor. The resolution test target replaced the sample and was illuminated by a non-coherent source (white light). The test target used in transmission is an optical glass substrate with clear lines on an opaque background. Figure 3.13 illustrates the selected test target, that has dimensions of $50 \times 50 \times 1,5 \mathrm{~mm}$, and Table 3.2 presents the specifications for each element. The maximum resolution for the test target used is 228.10 line pairs per millimeter, which equates to roughly $4.4 \mu \mathrm{m}$ per line pair.

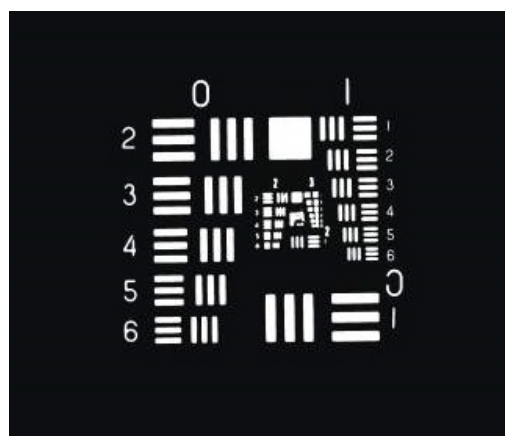

Figure 3.13: Newport's resolution test target recorded with the system using a non-coherent source.

Figure 3.14 shows the resulting image of the test target acquired with the system using a non-coherent light source. We can see that the group 2 , in the center of the test target, is still sharp. However, group 3 and group for ,the smallest pattern in the center, cannot be independently distinguished.

The last line pairs that is resolved is group 2, element 5 . When we look at element 6 , the line cannot not be distinguished. Therefore, according to the Table 3.2 , the resolution (lines $/ \mathrm{mm}$ ) is 6.35 , which was calculated by $(55)$ : 
Table 3.2: USAF-1951 resolution target standard specification (55). Line Pairs in millimeters

\begin{tabular}{ccccccccc}
\hline \hline \multirow{2}{*}{ Element Number } & \multicolumn{7}{c}{ Group Numbers } \\
\cline { 2 - 9 } & 0 & 1 & 2 & 3 & 4 & 5 & 6 & 7 \\
\hline 1 & 1.0 & 2.0 & 4.0 & 8.0 & 16.0 & 32.0 & 64.0 & 128.0 \\
\hline 2 & 1.1 & 2.2 & 4.5 & 9.0 & 18.0 & 35.9 & 71.8 & 143.7 \\
\hline 3 & 1.3 & 2.5 & 5.0 & 10.1 & 20.2 & 40.3 & 80.6 & 161.3 \\
\hline 4 & 1.4 & 2.8 & 5.7 & 11.3 & 22.6 & 45.2 & 90.5 & 181.0 \\
\hline 5 & 1.6 & 3.2 & 6.3 & 12.7 & 25.4 & 50.8 & 101.6 & 203.2 \\
\hline 6 & 1.8 & 3.6 & 7.1 & 14.2 & 28.5 & 57.0 & 114.0 & 228.1 \\
\hline Line pairs $/ \mathrm{mm}=\mathrm{LP}$ & \multicolumn{7}{c}{ Line width $(\mathrm{mm})=1 /(2 \mathrm{LP})$} \\
\hline Space width $(\mathrm{mm})=1 /(2 \mathrm{LP})$ & \multicolumn{7}{c}{ Line length $=5($ line width $)$} \\
\hline
\end{tabular}

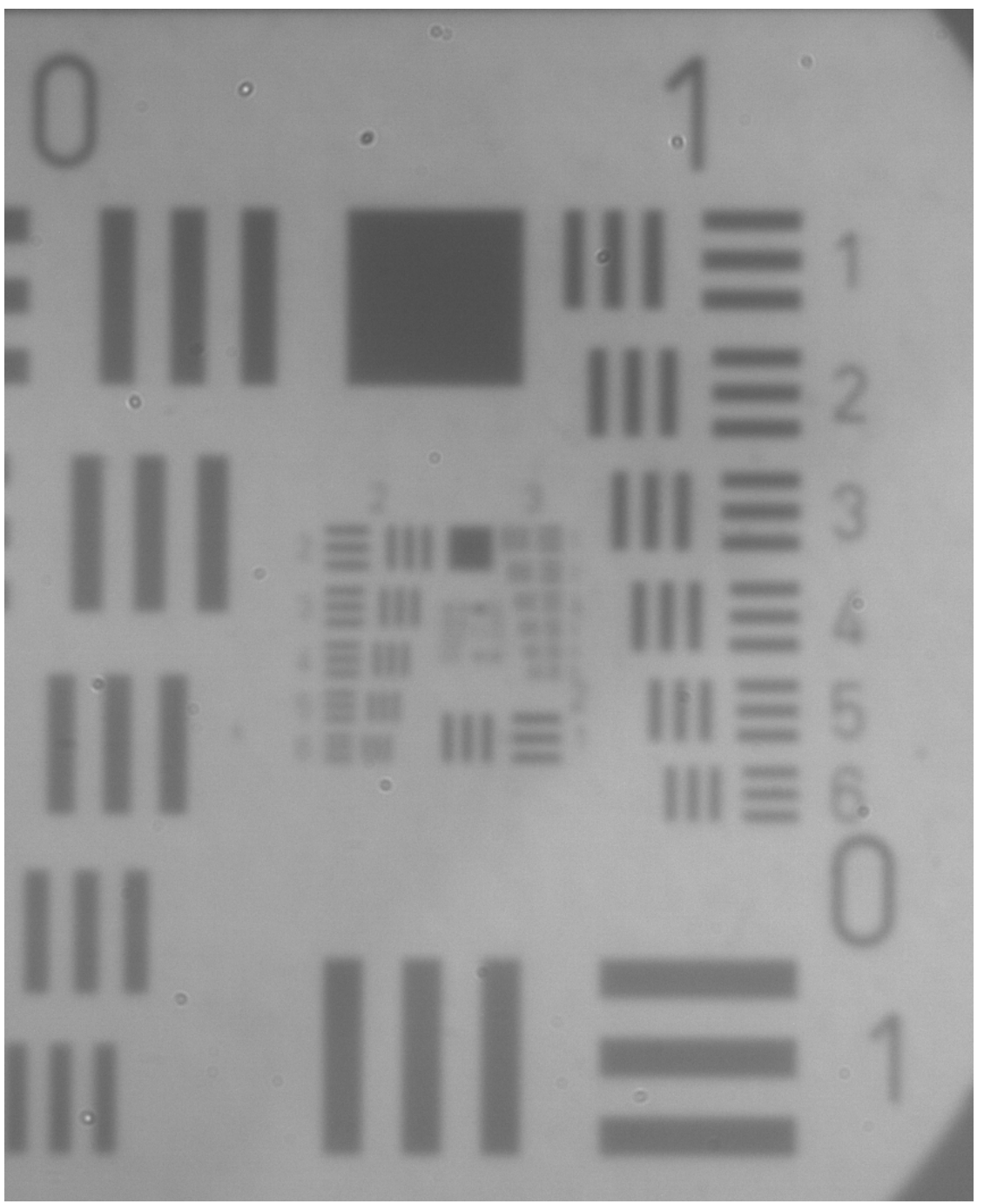

Figure 3.14: Image of a resolution test target recorded with the system using a non-coherent source.

$$
\text { Resolution }(\text { lines } / \mathrm{mm})=2^{\text {Group }+\frac{\text { Element-1 }}{6}}
$$

The CCD camera has a pixel size of $5.5 \mu \mathrm{m}$ by $5.5 \mu \mathrm{m}$ and the maximum 
size of the images acquired are 2048x1088 pixels. The acquisition process was automatized as to appropriately synchronize camera, transducer movement and AOM modulation. The camera is controlled by a MATLAB ${ }^{\circledR}$ program that also activates the function generator responsible to modulate the AOM and control the piezoelectric transducer. The program sets the modulation of the AOM and the sine waveform that runs the transducer, all synchronized with the camera to capture an image at the right moment of the stroboscopic pulse.

\subsection{1}

\section{Phase analysis}

Reconstruction of the phase variation due to object deformation is essencial in the process of making quantitative measurements with shearography. We now discuss the spatial-carrier technique, the method of choice to determine the phase distribution using a single recording. Compared to the temporalcarrier technique, a preferred method for most shearography applications, the spatial-carrier technique presents two significant advantages(56):

- The need of only one interferogram whereas the temporal-carrier demands several interferograms recorded at different times;

- No device needed to generate a carrier while the temporal-carrier techniques calls for a phase-shifting device.

In the spatial-carrier method, a carrier frequency is introduced to the intensity distribution, which will separate the phase from the background information in the frequency domain. The Fourier-transform method of fringe pattern analysis(57) is, then, applied to the resulting image to isolate and calculate the phase distribution.

The following equation illustrates the form of an interferogram generated by the spatial-carrier method:

$$
I(x, y)=b(x, y)+I_{0}(x, y) \cos \left[2 \pi f_{0} x+\Phi(x, y)\right]
$$

where $b(x, y)$ is the background intensity, $I_{0}(x, y)$ is the fringe amplitude, $\Phi(x, y)$ is the phase to be determined, and $f_{0}$ is the carrier frequency introduced by the tilted reference wavefront. We assume the component of the carrier frequency as $f_{0}=\sin \theta / \lambda$, introduced by the tilted mirror of angle $\theta$, and since we consider only small angles, it can be approximated to $f_{0}=\theta / \lambda$.

Considering that the spatial variations of $I_{0}(x, y), b(x, y)$, and $\Phi(x, y)$ are slow compared to $f_{0}$, the Fourier spectra are separated by the carrier frequency $f_{0}$, as shown by Figure 3.15. 


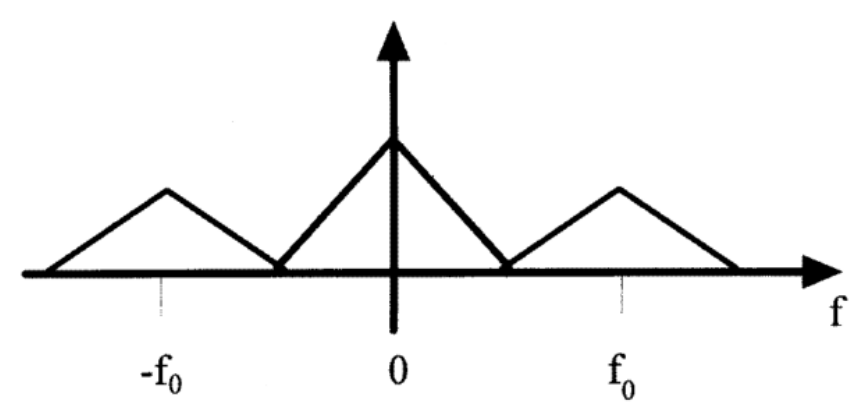

Figure 3.15: Fourier spectrum of the interferogram. The zero-order term is separated $f_{0}$ from the \pm 1 terms. Figure from (44)

Equation 3-20 and Equation 3-21 are the two incident wavefronts arriving at the CCD camera.

$$
u_{1}(x, y)=\left|u_{1}(x, y)\right| \exp \{i \phi(x, y)\}
$$

$$
u_{2}(x, y)=\left|u_{1}(x+\Delta x, y+\Delta y)\right| \exp \left\{i \phi(x+\Delta x, y+\Delta y)+2 \pi f_{0} x\right\}
$$

The intensity detected by the CCD sensor of the interference between $u_{1}$ and $u_{2}$ is:

$$
\begin{array}{r}
I=u_{1} \overline{u_{1}}+u_{2} \overline{u_{2}}+u_{1} \overline{u_{2}}+u_{2} \overline{u_{1}} \\
=\left|u_{1}(x, y)\right|^{2}+\left|u_{1}(x+\Delta x, y+\Delta y)\right|^{2} \\
+\left|u_{1}(x, y)\right|+\left|u_{1}(x+\Delta x, y+\Delta y)\right| \\
\times \exp \left\{i \phi(x, y)-\phi(x+\Delta x, y+\Delta y)-2 \pi f_{0} x\right\} \\
+\left|u_{1}(x, y)\right|+\left|u_{1}(x+\Delta x, y+\Delta y)\right| \\
\times \exp \left\{i \phi(x, y)-\phi(x+\Delta x, y+\Delta y)-2 \pi f_{0} x\right\}
\end{array}
$$

As follows, we apply the Fourier Transform to isolate and calculate the phase distribution.

$$
\mathcal{F}\{I\}=U_{1} \otimes \bar{U}_{1}+U_{2} \otimes \bar{U}_{2}+U_{1} \otimes \bar{U}_{2}+U_{2} \otimes \bar{U}_{1}
$$

where $U_{1}=\mathcal{F}\left\{u_{1}\right\}$ and the bar represents the complex conjugate and $\otimes$ is the convolution operation. The phase information is encoded in the last two convolution terms of Equation 3-23 separated by $f_{0}$ from the zero order term. In a speckled environment, the amplitudes and relative phases are changing randomly as a function of the position, however, the mean speckle size defines the area where they remain constant. Accordingly, the next step is the filtering process to remove the carrier frequency. We achieve this by applying an inverse Fourier transform to the isolated and re-centered term: 


$$
\Delta \phi-2 \pi x f_{0}=\arctan \left(\frac{\Im\left[u_{1} \overline{u_{2}}\right]}{\Re\left[u_{1} \overline{u_{1}}\right]}\right)
$$

Following the loading process where the object is deformed, the optical path changes. Equation 3-25 and Equation 3-26 represent the waveforms after a deformation is applied:

$$
\begin{gathered}
u_{1}^{\prime}(x, y)=\left|u_{1}^{\prime}(x, y)\right| \exp \left\{i \phi^{\prime}(x, y)\right\} \\
u_{2}^{\prime}(x, y)=\left|u_{1}^{\prime}(x+\Delta x, y+\Delta y)\right| \exp \left\{i \phi^{\prime}(x+\Delta x, y+\Delta y)+2 \pi f_{0} x\right\}
\end{gathered}
$$

The same process described above is applied for these images. After the phase difference, we can extract the first order derivatives of the displacement in the direction of shear.

With all the images recorded, the processing to extract the phase information starts, as described in chapter 2. All the results acquired with this system will be presented in chapter 4 . 


\section{4 \\ Experimental results}

\section{1}

\section{LOFI experimental results}

As it was discussed in section 3.1, with a moving target with frequency superior to a few kilohertz, the method to determine the phase $\Phi(x, y, t)$ is the acquisition of the temporal trace of the LOFI signal $S_{L O F I}$ and calculating its Hilbert Transform using Equation 3-10. This processing allows to obtain the vibration function of the sample and, therefore, access its amplitude and phase over time.

With the LOFI system presented in chapter 3, we ran three different experiments. First, we present and evaluate the behavior of a piezoelectric transducer vibrating at $40 \mathrm{kHz}$. Then, high frequency measurements are discussed, with a linear gain compensation was applied. For smaller vibrations, we need a non-linear gain compensation. We present the experimental results in this chapter, while the mathematical base and the numerical simulations of the non-linear gain are discussed in Appendix A.

Some changes were made to the detection system of the LOFI in comparison to the image acquisitions shown in section 3.1. We now acquire vibration frequencies for each given vibration amplitude. The lock-in amplifier, that is part of the detection system depicted in Figure 3.1, now works as a detector and filter.

\subsection{1}

\section{Vibration measurements at $40 \mathrm{kHz}$}

Figure 4.1 shows the vibration measured on the surface of a piezoelectric transducer vibrating at $40 \mathrm{kHz}$ frequency with the burst lasting for 100 periods. These results were acquired with the system presented in Figure 3.1. The number of points were $N=500000$, a sampling rate of $50 \mathrm{MHz}$, acquisition time of $16 \mathrm{~ms}$ with burst lasting $2.5 \mathrm{~ms}$, the output power at the sample is $20 \mu \mathrm{W}$ and the carrier frequency $F_{0}=1.6 \mathrm{MHz}$.

The red curve is the envelope of the signal sent to the transducer (duration of a burst) and the blue curve is the vibration measured over time 
with the LOFI set up presented on Figure 3.1. It is possible to observe the response time of the piezoelectric transducer from the later measurement, where the noise is of the order of 3 angstroms. This noise corresponds to the standard deviation measured at the absence of the burst (time inferior to $4 \mathrm{~ms}$ on Figure 4.1).

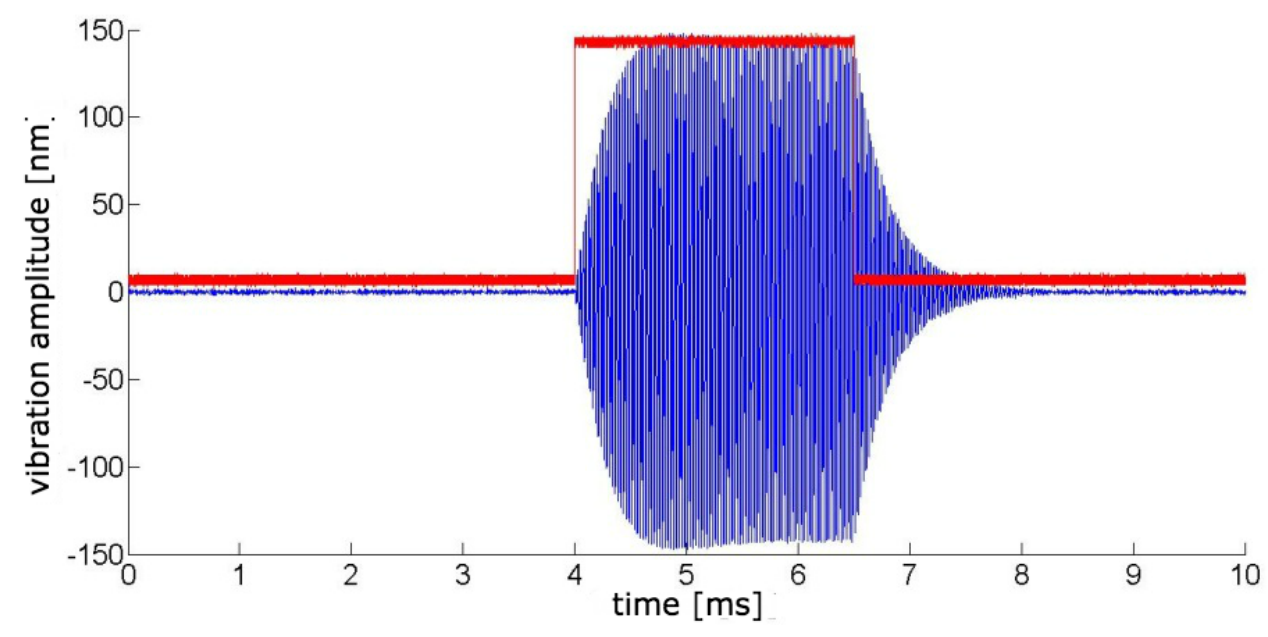

Figure 4.1: Results for a piezoelectric transducer with a burst of 100 periods at $40 \mathrm{kHz}$.

To illustrate the process mentioned in section 2.3, Figure 4.2 presents a one dimension Fourier Transform of the acquired signal with the same experimental conditions mentioned above for one $40 \mathrm{kHz}$ pulse. The peaks of the Bessel Function are visible on the blue curve, while the green curve represent the linear gain.

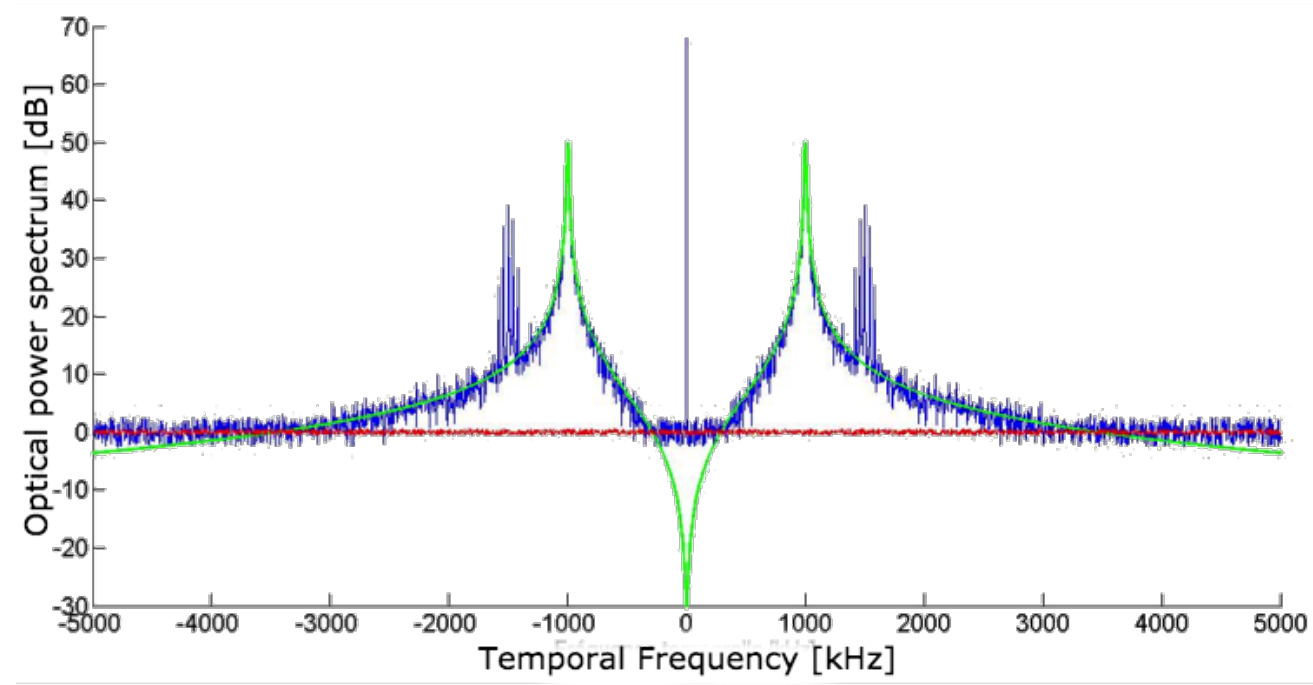

Figure 4.2: Fourier Transform of the signal with the peaks of the Bessel Function. The green curve shows the linear gain. 
The next step is to filter the order that has the vibration information, as discussed previously. Figure 4.3 shows the resulting filtered +1 order curve from the Fourier Transform depicted in Figure 4.2.

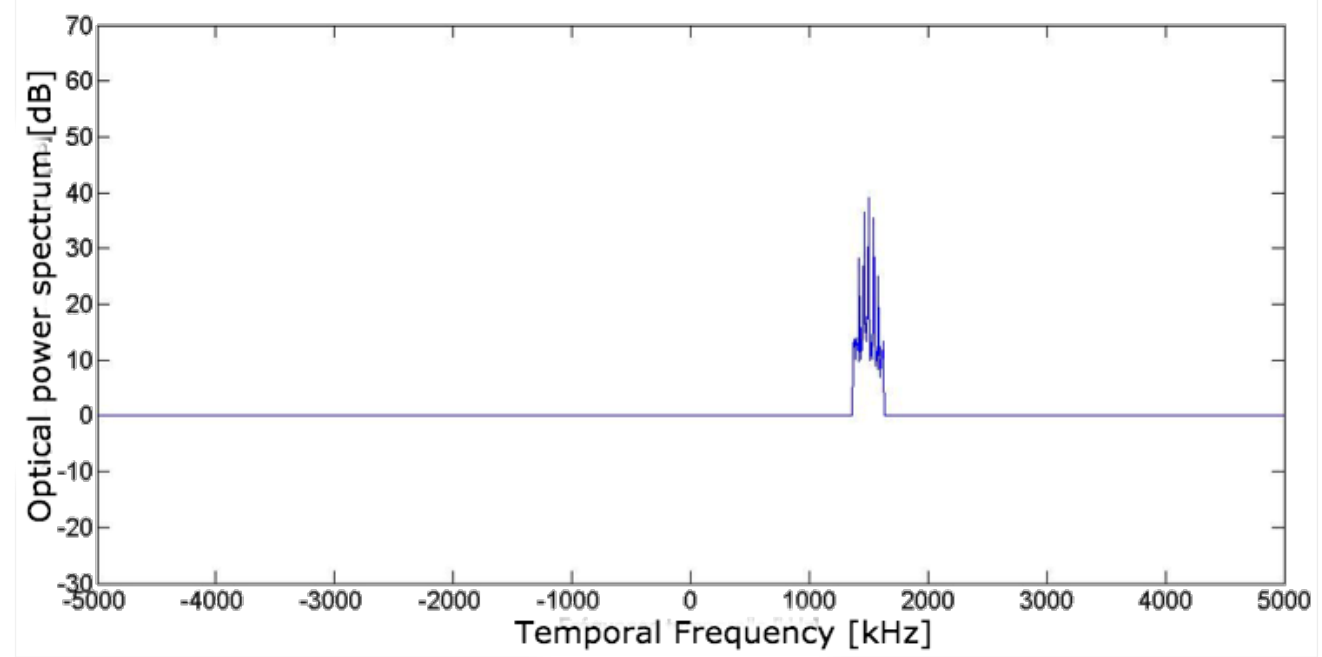

Figure 4.3: Resulting curve from filtering the +1 order of the Fourier Transform shown in Figure 4.2.

After filtering, we transform the signal back to the time domain and as it is illustrated in Figure 4.4, recover the vibration sent to the piezoelectric transducer. The next stage of our experimental tests is to acquire the same results, but now in two dimensions.

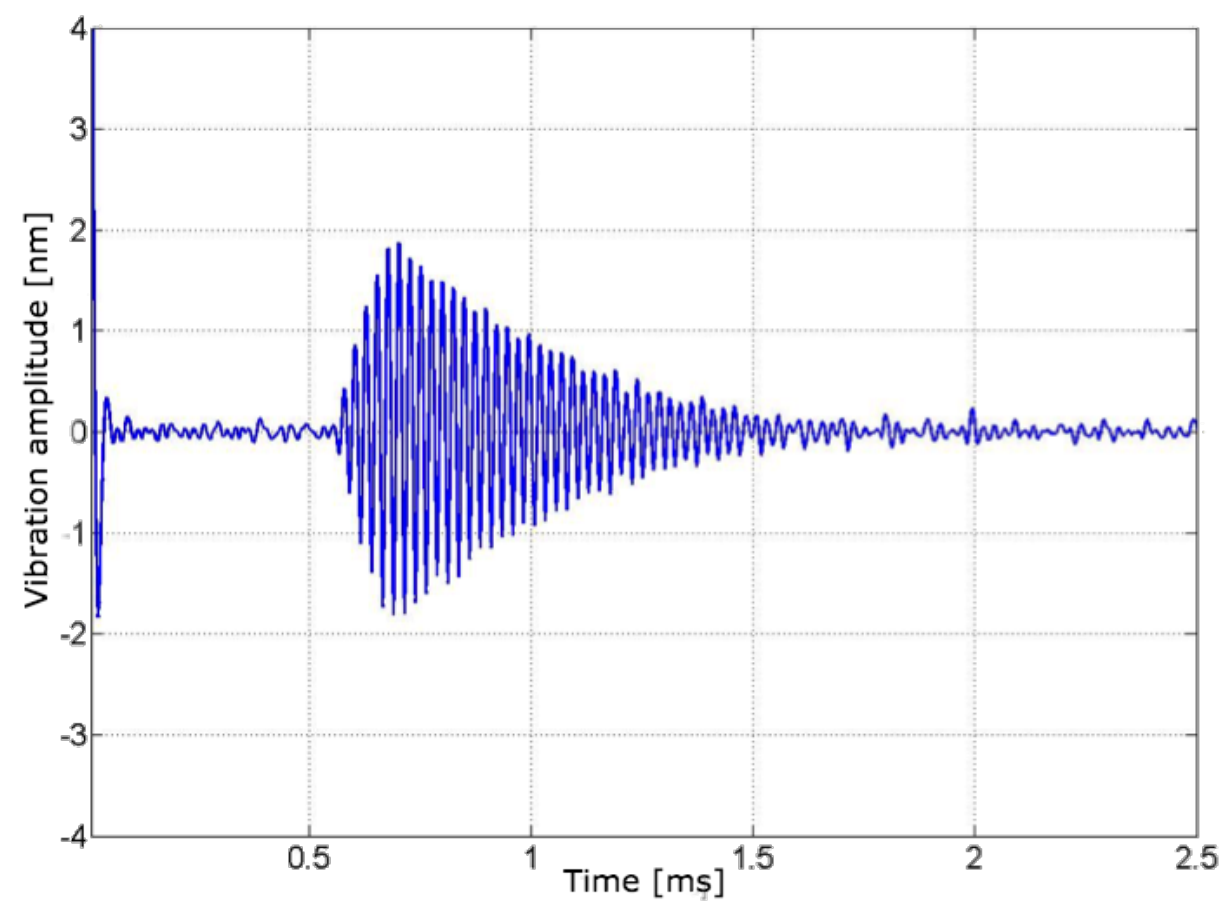

Figure 4.4: Resulting 1D filtered signal for one burst at $40 \mathrm{kHz}$. 
With the help of galvanometer mirrors, we scan the piezoelectric transducer with the laser beam (focalized on the target) in order to obtain the vibration in different points (pixels), the same measurement done in Figure 4.1. In each measured point, a vibration frequency was obtained at a given vibration amplitude. A dynamic mapping (video) of the vibrations on the surface can also be acquired. Each temporal trace is made of a pixel from the video of the vibration. Figure 4.5 presents two static moments of a video acquisition of the surface of a piezoelectric sensor vibrating at $40 \mathrm{kHz}$ with amplitude of $30 \mathrm{~nm}$. These reconstructed maps were acquired outside the transient regime of the sensor (between 5 and $6 \mathrm{~ms}$ in Figure 4.5). It is possible to observe that the transducer does not move in a piston motion.

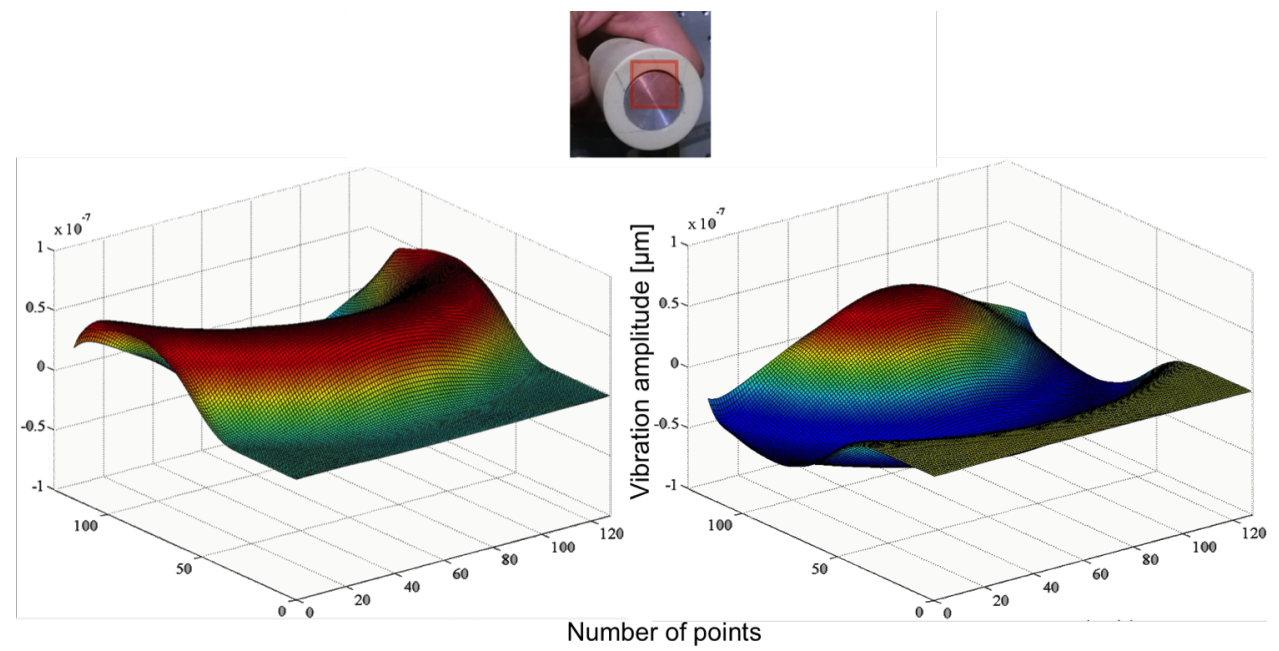

Figure 4.5: Excerpt from the video of the vibrating surface of the piezoelectric sensor (30 nm vibration at $40 \mathrm{kHz}$ ). On the top, the blue square shows the region evaluated.

Figure 4.6 is a plot of the measurement of a ultrasound wave of 2.25 $\mathrm{MHz}$ frequency where the spectrum of the LOFI signal in the presence of an ultrasound wave can be observed. The ultrasound wave was generated inside a glass tank full with water and it is propagating towards the surface. The acoustic transducer is focused at the water surface. The vibration measurement is done at the water surface, with the laser beam LOFI focalized at the surface. The carrier frequency shift $F_{0}$ is $3.2 \mathrm{MHz}$ (peak $F_{0}$ in Figure 4.6 ) and $2.5 \mathrm{~ms}$ bursts.

The vibration of the surface translates into the appearance of the lateral stripes on each side of the carrier of frequency $F_{0} \pm n . f_{v}$. Only the stripe of -1 order at $0.95 \mathrm{MHz}$ frequency is visible in the figure. The other stripes are outside the measured spectrum and certainly below the detector noise because they have short amplitude. In fact, they are distant from the resonance frequency $F_{R}=0.75 \mathrm{MHz}$ of the laser and hence do not benefit from the 
amplification $G_{L O F I}$. After correcting the gain, the amplitude ratio of these peaks informs us about the vibration amplitude.

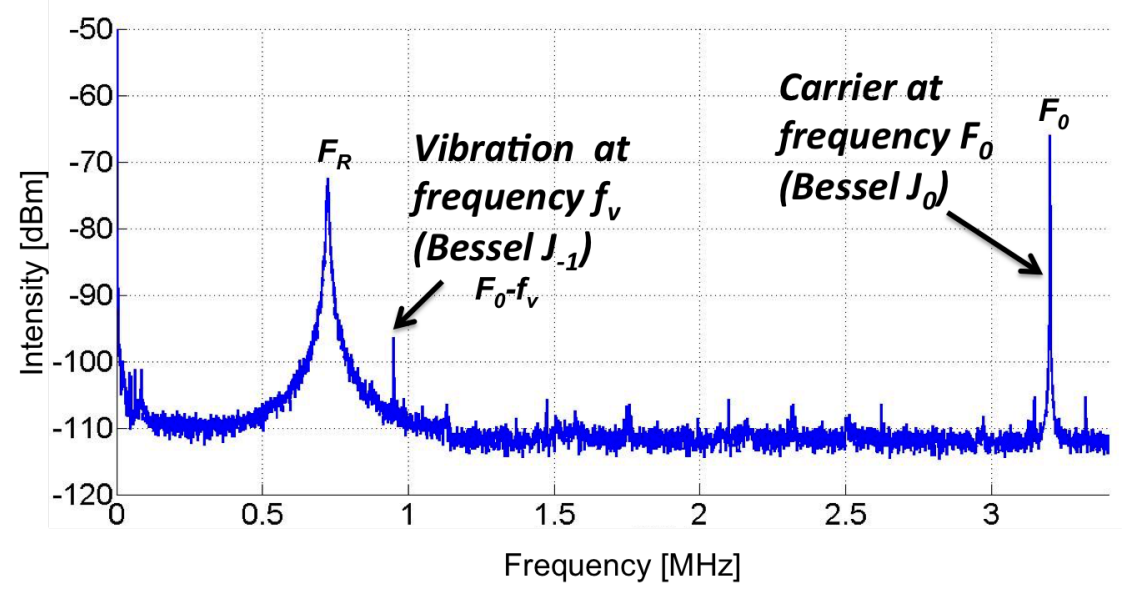

Figure 4.6: Noise spectrum of the LOFI applied to high frequency vibrometry.

To access the vibration amplitude in the case presented above, we compensated the gain. This is a correction is the linear gain described in Equation 3-6. If we intend to access small vibrations or observe these vibrations on samples with low reflective surfaces (e.g. the skin) in our photoacoustic application, we have to raise the reinjection into the LOFI. Raising $F_{0}$ involves non linear effects. Therefore, to access small vibrations amplitude we need to compensate the non linear form of the gain. This will be discussed in the next section.

\subsection{2}

\section{Non-linear gain compensation}

As mentioned above, for small vibration amplitudes we need to compensate the non linear gain. All mathematical discussion and the related numerical analysis is presented in Appendix A. In this section, we concentrate on the experimental results for the non-linear compensation.

To demonstrate that the signal processing can be applied to any kind of vibrations, we have experimentally measured transient-harmonic vibrations with the LOFI setup (Figure 4.7). Experimentally, the laser is set to an output power of $40 \mathrm{~mW}$ with a relaxation frequency of $F_{R}=1.17 \mathrm{MHz}$ and the carrier frequency (controlled by the two AOMs) is tuned to $F_{0}=1.24 \mathrm{MHz}$ (meaning $\left.F_{0} / F_{R}=1.06\right)$. Experiments have been made for 3 different amounts of optical feedback. The strength of the optical feedback is controlled by adjusting the conversion efficiency of the two AOMs.

In Figure 4.7, the power of optical feedback is characterized by the laser power impacting the target: $1.8 \mu W$ for the top row, $5.6 \mu W$ for the middle 
row, and $14.9 \mu \mathrm{W}$ for the bottom row. These values correspond to a relative increase of the effective feedback reflectivity $R_{e}$ by a factor of $\times 9.7$ between the first two values and by a factor $\times 7.1$ between the second and the third.
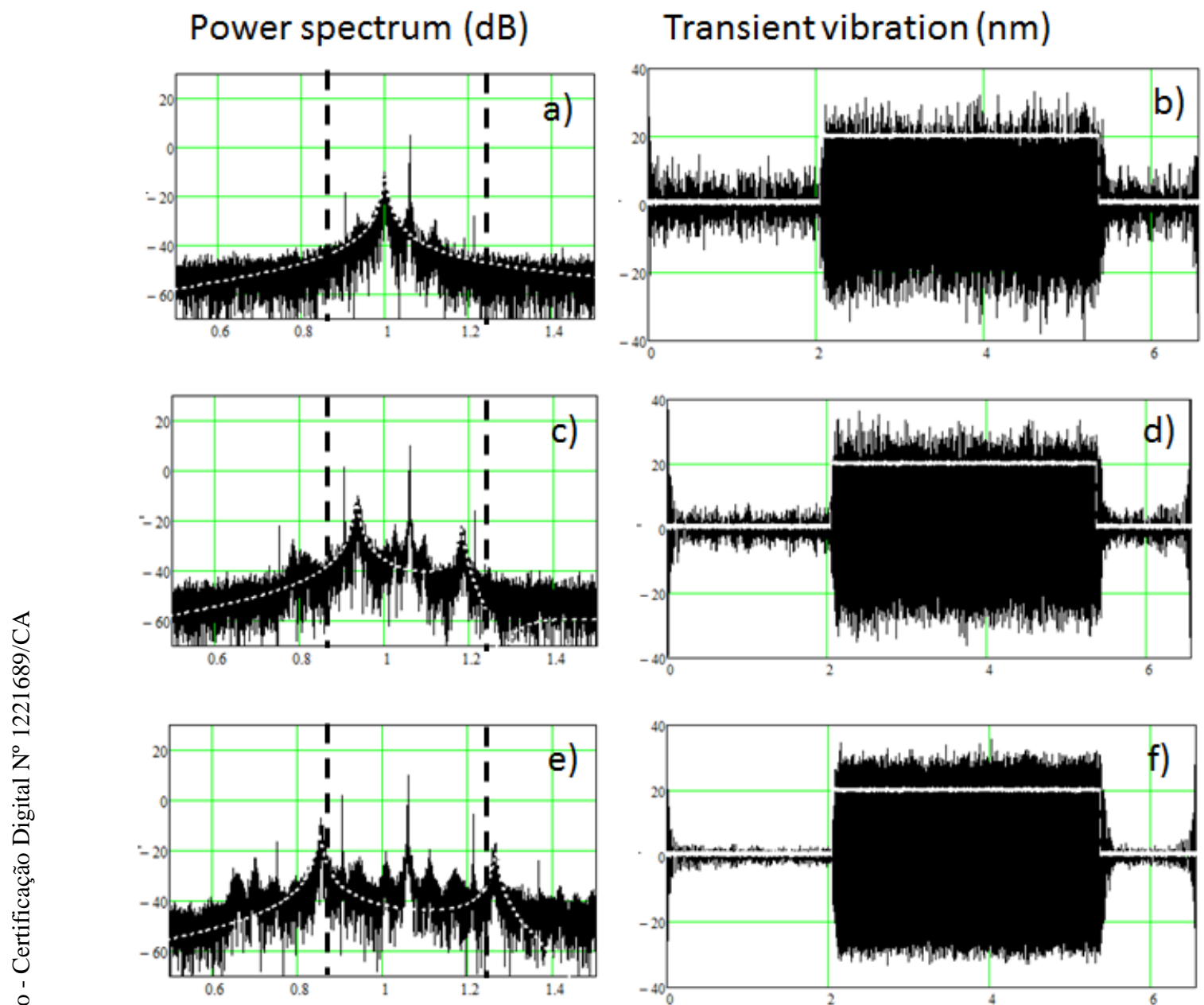

Frequency $(\mathrm{MHz})$

Time (ms)

Figure 4.7: Detection of a transient-harmonic vibrations with an oscillation frequency of $F_{a}=180 \mathrm{kHz}$ and a vibration amplitude of $d_{a}=28 \mathrm{~nm}$. The measurements are made for $F_{0}=1.24 \mathrm{MHz}$ and an increasing amount of optical feedback characterized by the laser power impacting the vibrating PZT. Top row (a, b): low feedback $(1.8 \mu W)$; Middle row (c, d): intermediate feedback $(5.6 \mu W)$; Bottom row (e, f): strong feedback $(14.9 \mu W)$. The left column (a, c, e) shows the RF power spectra, where the dashed white-lines show the shape of the non-linear gain used for the signal processing. The signal processing is made with a band-pass filter $\Delta F_{e}=450 \mathrm{kHz}$ (corresponding to the dashed vertical black-lines). The right column (b, d, f): shows a comparison between the vibration extracted from the signal processing and the time gate when the PZT is triggered (solid white lines). (58)

The transient-harmonic vibration generated by the piezoelectric translation stage is composed of harmonic oscillations with a frequency of $F_{a}=$ 
$180 \mathrm{kHz}\left(F_{R} / 6.5\right)$ within a time gate of $3.3 \mathrm{~ms}$, therefore 600 cycles, starting after a time delay of $2 \mathrm{~ms}$. The total recording time is $6.6 \mathrm{~ms}$ with a sampling rate of $1 \times 10^{7}$ samples $/ \mathrm{s}$. For the signal processing the frequency bandwidth is $\Delta F_{0}=450 \mathrm{kHz}$ around $F_{0}$.

Figure 4.7 shows that quantitative vibration measurements can be experimentally extracted from the non-linear laser dynamics. The left column shows how the gain is modified when the amount of optical feedback increases. The modification of the non-linear gain is induced by the modification of the carrier amplitude. Starting with a linear gain (plot a), the gain becomes more and more non-linear when the optical feedback increases (plots b and c).

In agreement with equation A-25a, it can be observed that, for the nonlinear gain (Figure 4.7c and e), the frequency distance between the two maxima (located symmetrically on both side of the carrier frequency) increases. And, in agreement with equation A-25b, it can also be observed that the amplitude of the maximum located on the right side of the carrier frequency increases.

The plots on the left column of Figure 4.7 show that the modulation amplitude at the carrier frequency can saturate when the optical feedback becomes too strong. Indeed, Figure 4.7c and e show that the amplitude of the carrier is roughly constant, although the amount of optical feedback is increased 7.1 times. Despite this saturation, the images on the right column of Figure 4.7 show that, regardless of the shape of the gain (linear or nonlinear), the transient-harmonic vibration is recovered with an accurate time delay, characterized by the trigger trace superimposed. It should be noted that the presented time traces are an average on ten acquisitions. By looking more precisely near the rising and falling edges, it can also be observed the transient time response of the piezoelectric transducer.

When the amount of optical feedback increases, a small increase of the mean value of the stationary vibration amplitude (21 $\mathrm{nm}, 25 \mathrm{~nm}$ and $28 \mathrm{~nm}$ ) can be observed, while there's a small decrease of the vibration noise $(6 \mathrm{~nm}, 3 \mathrm{~nm}$ and $1 \mathrm{~nm}$ ). The amplitude of the vibration noise has been determined during the starting time delay (i.e. before the starting of the harmonic vibrations). For a given detection bandwidth, this reduction of the vibration noise could be qualitatively explained by the increase of the carrier signal, coupled simultaneously with a modification of the laser noise power spectrum induced by the optical feedback, in the vibration frequency range.

For comparison, the vibration amplitude value measured at low frequency $(150 \mathrm{~Hz})$ with a lock-in amplifier gives $28 \mathrm{~nm}$. So, taking into account the noise, the obtained results are in relative good agreement and a better value can be obtained for the strongest feedback $(28 \pm 1 \mathrm{~nm})$ where the non-linear 
gain compensation is necessary.

\section{2}

\section{Stroboscopic Mach-Zehnder experimental results}

As for the LOFI, the experimental results were obtained from a piezoelectric transducer working at a frequency of $40 \mathrm{kHz}$, depicted in Figure 4.8. As described in chapter 3, the source is a $15 \mathrm{~mW}$ He-Ne laser, but the power at the surface of the sample is around $3.5 \mathrm{~mW}$. To record the images, we use the CCD sensor previously described, with a exposure time initially set at $7500 \mu \mathrm{s}$. The AOM was modulated at $40 \mathrm{kHz}$ and the stroboscopic pulse duration was $1 \mu s$.

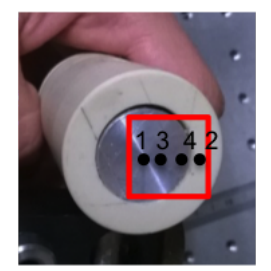

Figure 4.8: Piezoelectric transducer analyzed by the Stroboscopic MachZehnder. The red square indicates approximately the imaged area.

The approximately area analyzed is illustrated by the red square on the image. The transducer's surface is metallic and diffusing and, consequently, presents a significant roughness. This roughness will be translated in the form of a speckle pattern resulting from a coherent illumination. The moving part of the transducer has a diameter of $1.9 \mathrm{~cm}$ and the static part has $0.6 \mathrm{~cm}$ width around the piezoelectric plate. This is the same transducer used to acquire the results presented in section 4.1, allowing to compare both systems in chapter 5 .

A sequential acquisition of images allows us to evaluate the complete movement of the piezoelectric transducer. To properly analyze the area studied, we determined 4 different amplitude positions, as indicated by the numbers in Figure 4.8:

- 1 - Center of the transducer;

○ 2 - Edge (no vibration a priori);

- 3 - Intermediary position between the center and the edge corresponding to a weak amplitude of vibration; and

○ 4 - Intermediary position between the center and the edge corresponding to a strong amplitude of vibration.

These positions were determined based on the observation from the images acquired that there's no piston movement. This means The vibration ampli- 
tude depends on the position considered on the surface of the piezoelectric transducer, which will affect its movement.

Figure 4.9 shows 4 images of the speckle pattern acquired from the transducer cited above with the stroboscopic Mach-Zehnder described in section 3.4. The top two images ( $a$ and $b$ ) show the images acquired from the object arm and the reference arm, respectively. These images were obtained by blocking one arm of the interferometer, for example, when recording the signal from the transducer's surface, the reference arm was blocked and vice-versa. Figure $4.9 \mathrm{c}$ shows the interference pattern between both arms, while image d) is a zoom of the square indicated on image $c$ ).

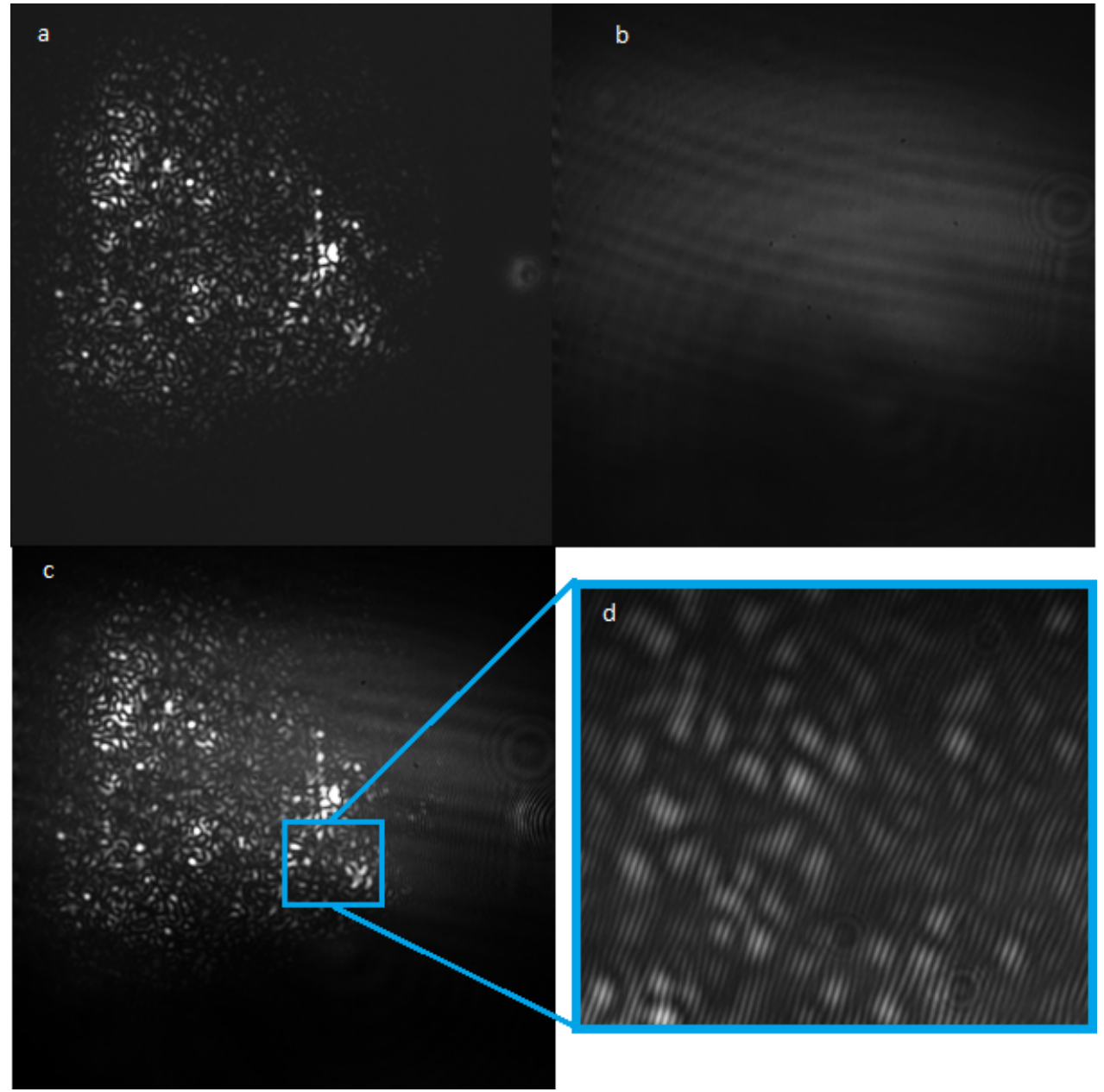

Figure 4.9: Speckle pattern of the piezoelectric transducer's surface. a)Signal from the object arm; b) Signal from the reference arm; c) Interference pattern between both arms; and d) Zoomed area of the interference pattern presented on image $\mathrm{c}$ ).

On the object arm, the speckle pattern is generated by the diffusing aspect of the transducer's surface. From the recorded images it can be assumed that on the reference arm the wave is not completely plane, however, there's a uniform intensity along the beam. When the signal from both arms are 
combined, the interference pattern appears as expected. We can identify around 3 or 4 fringes in one speckle. The goal is to have several fringes in one speckle, its size being adjusted with a diaphragm placed in the object arm. The number of fringes in one speckle is managed by the angle of the reference arm's mirror, being this ratio crucial for the the separation of the order of the Fourier Transform, as detailed in chapter 2.

It can be observed some noise in Figure 4.9b. The diffraction spots, due to dust on the optical elements, and a parasite interference caused by the protective window of the CCD camera. This noise sources, however,do not extensively affect the results our results, as it can be seen in the Fourier Transform of the results, depicted in Figure 4.10.

We apply the Fourier Transform to the 3 cases shown above in order to acquire their spatial spectrum, as depicted in Figure 4.10. They are in agreement with the expected results from each arm of the interferometer (Figure 4.10a for the speckle pattern of the imaged sample and Figure 4.10b for the reference beam). That is, a typical spectrum of a speckle pattern and of a quasi plane wave. Also, the spectrum of the interference is in conformity with what is expected, pictured in Figure 4.10c. It is on this resulting Fourier Transform of the interference pattern that we continue the process to determine the phase, as described in section 2.3.
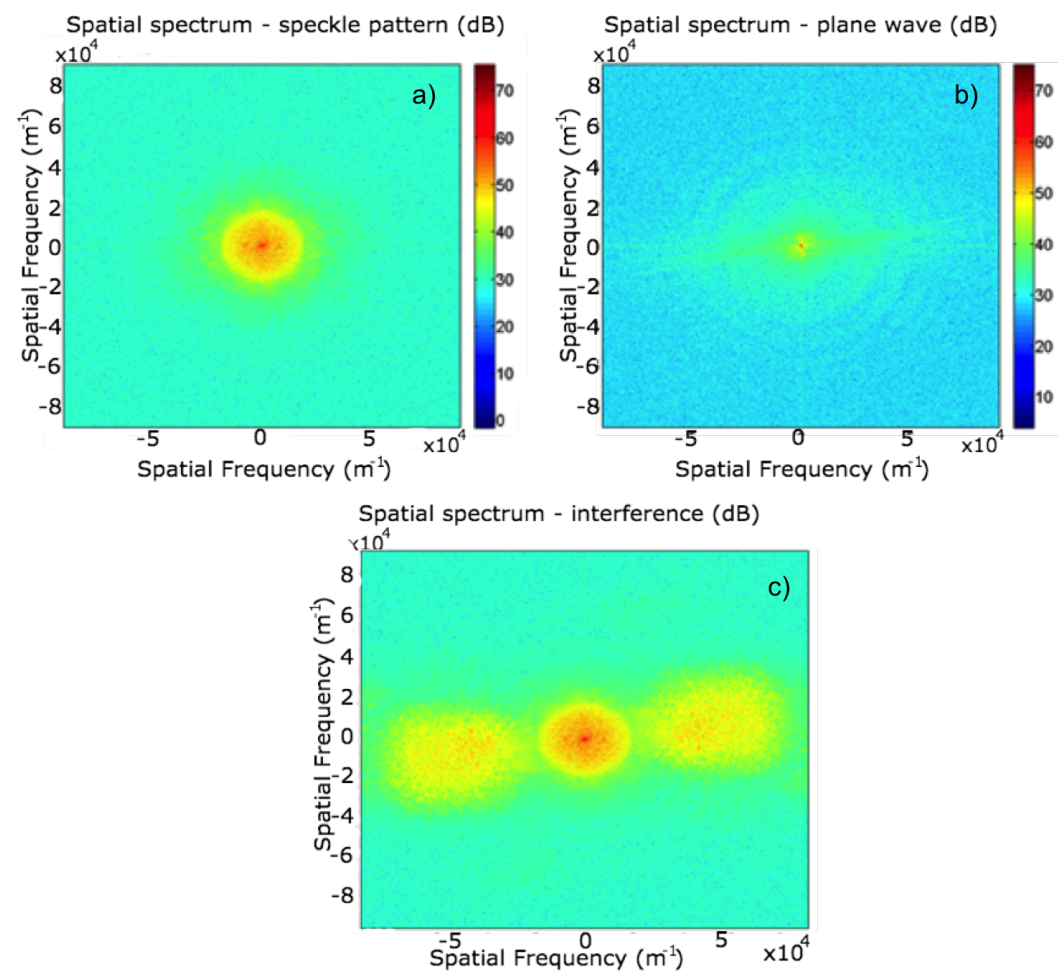

Figure 4.10: Spatial spectrum of: a) speckle pattern of the imaged sample; b) reference beam; and c) interference pattern. 
The mirror's tilt on the reference arm is adjusted in a manner that allows the separation of the orders of the Fourier Transform. This means controlling the number of fringes in a speckle, as discussed above. With this separation between the orders, detailed in chapter 2 , we can carry out the processing to determine the phase, i.e. the vibration at a given instant.

One example of the processing described above is shown in figures 4.11 and 4.12. After applying a Fourier Transform to the image map (Figure 4.11), the +1 order containing the deformation information is filter from Fourier map, as illustrated in Figure 4.12.

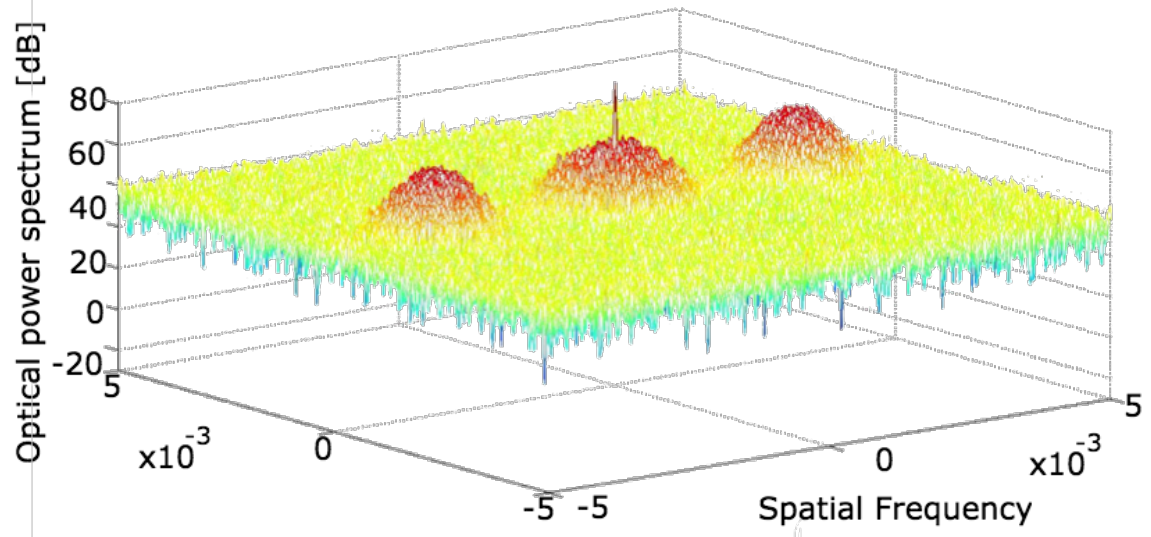

Figure 4.11: 3D plot of a Fourier Transform of one image map.

After filtering, we apply the inverse Fourier Transform to return to the time domain and carry on with the phase difference between the reference image and the following instant acquired and the necessary phase unwrapping.

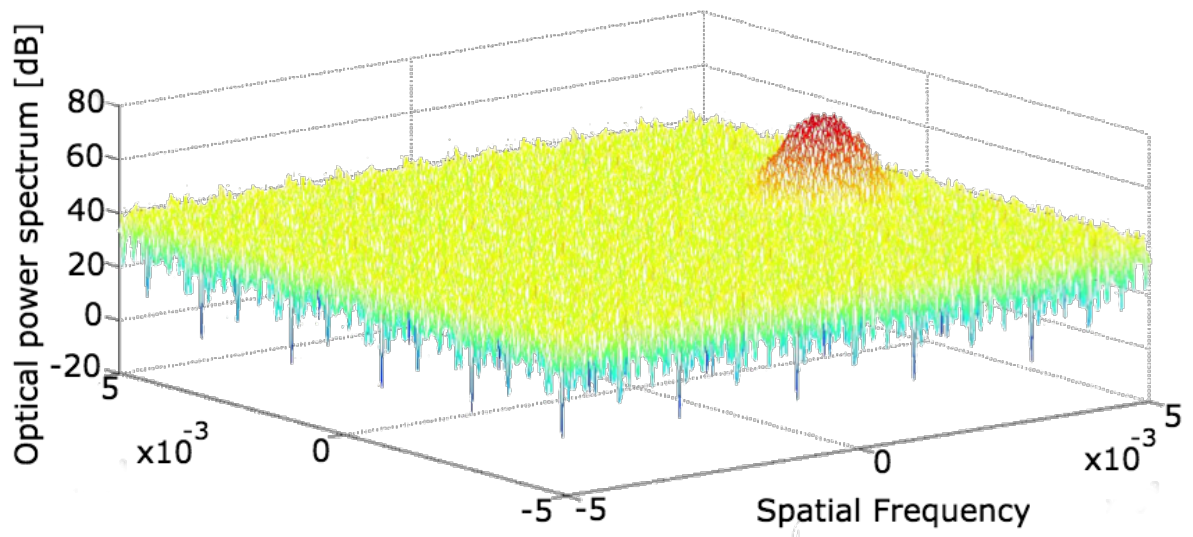

Figure 4.12: Resulting curve from filtering the +1 order of the Fourier Transform shown in Figure 4.11.

Figure 4.13 presents four 3D images of the processed phase. These images are different moments on the sequential acquisition of the vibration of the 
transducer. To acquire these images, the trigger of the piezoelectric transducer, the trigger and delay of the stroboscopic pulse and the recording of the signal captured by the CCD sensor had to be synchronized. From these 4 images, we can observe the different amplitude positions previously defined. They will be important for the analysis of the results. We measured the same movement with the LOFI.
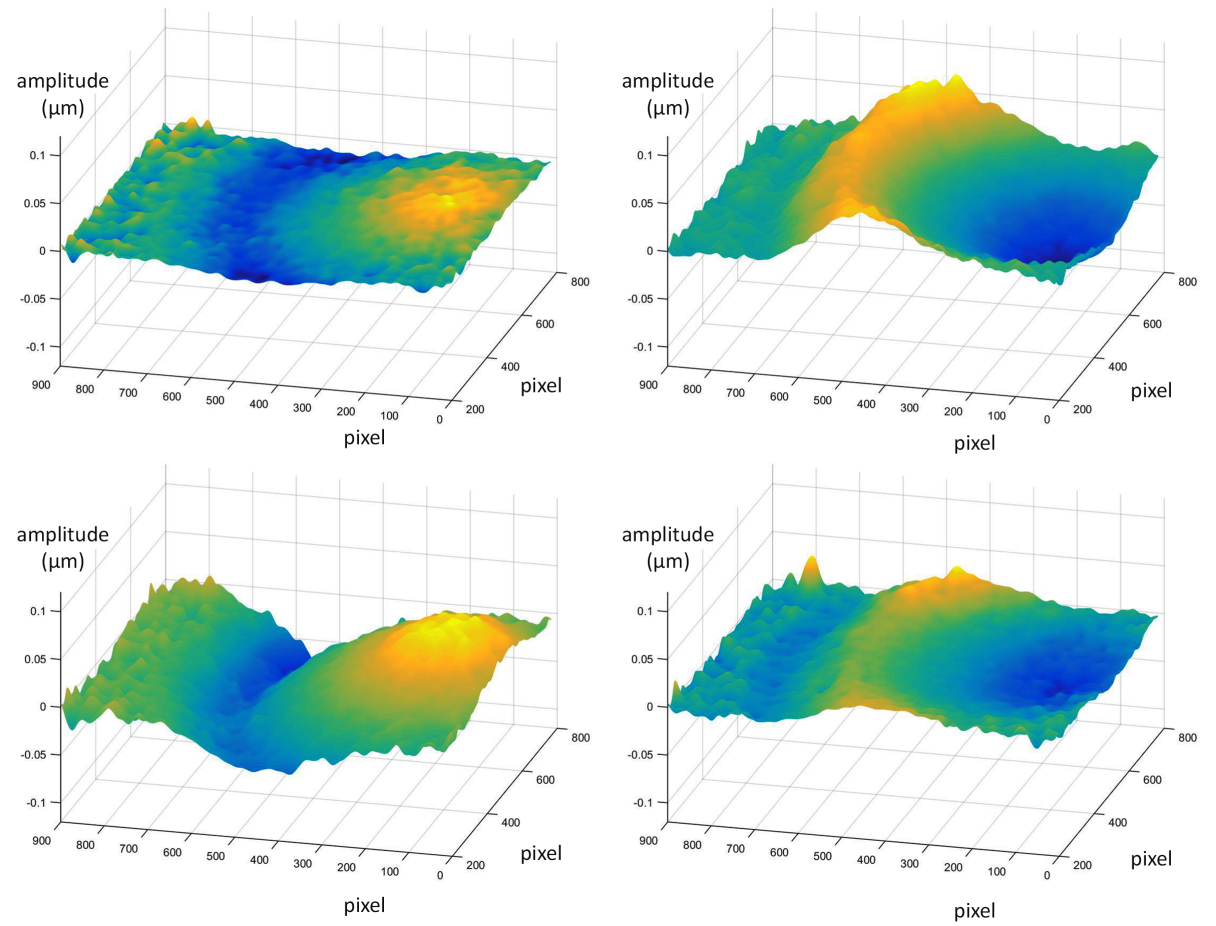

Figure 4.13: 3D phase images over a time period. Piezoelectric transducer vibrating at $40 \mathrm{kHz}$.

The phase and amplitude variations were determined over a period of time and then the result was fitted with a sinus function $f=a+b * \sin (w t+c)$, where $w=2 \pi \times 40 \mathrm{kHz}$. In Figure 4.14, we present the amplitude analysis over time period of $80 \mu \mathrm{s}$ for the 4 positions defined earlier. Figure $4.14 \mathrm{a}$ and $\mathrm{c}$ present high amplitude of vibration, but alternating in terms of maximums and minimums. Figure $4.14 \mathrm{c}$ presents the position of weak amplitude of vibration, with the maximum value being only $0.02 \mu \mathrm{m}$. Even though it still resembles a sine, Figure $4.14 \mathrm{~d}$ presents the amplitude of vibration of the edge of the transducer, which would be expected to present no vibration. This measurement noise is due to the vibration generated by the operation of the piezoelectric plate, corresponding to the wave seen in Figure 4.14d. For the measurement point situated at the edge of the transducer, no adjustment was necessary since there is no vibration.

The results are consistent with what would be expected from each 

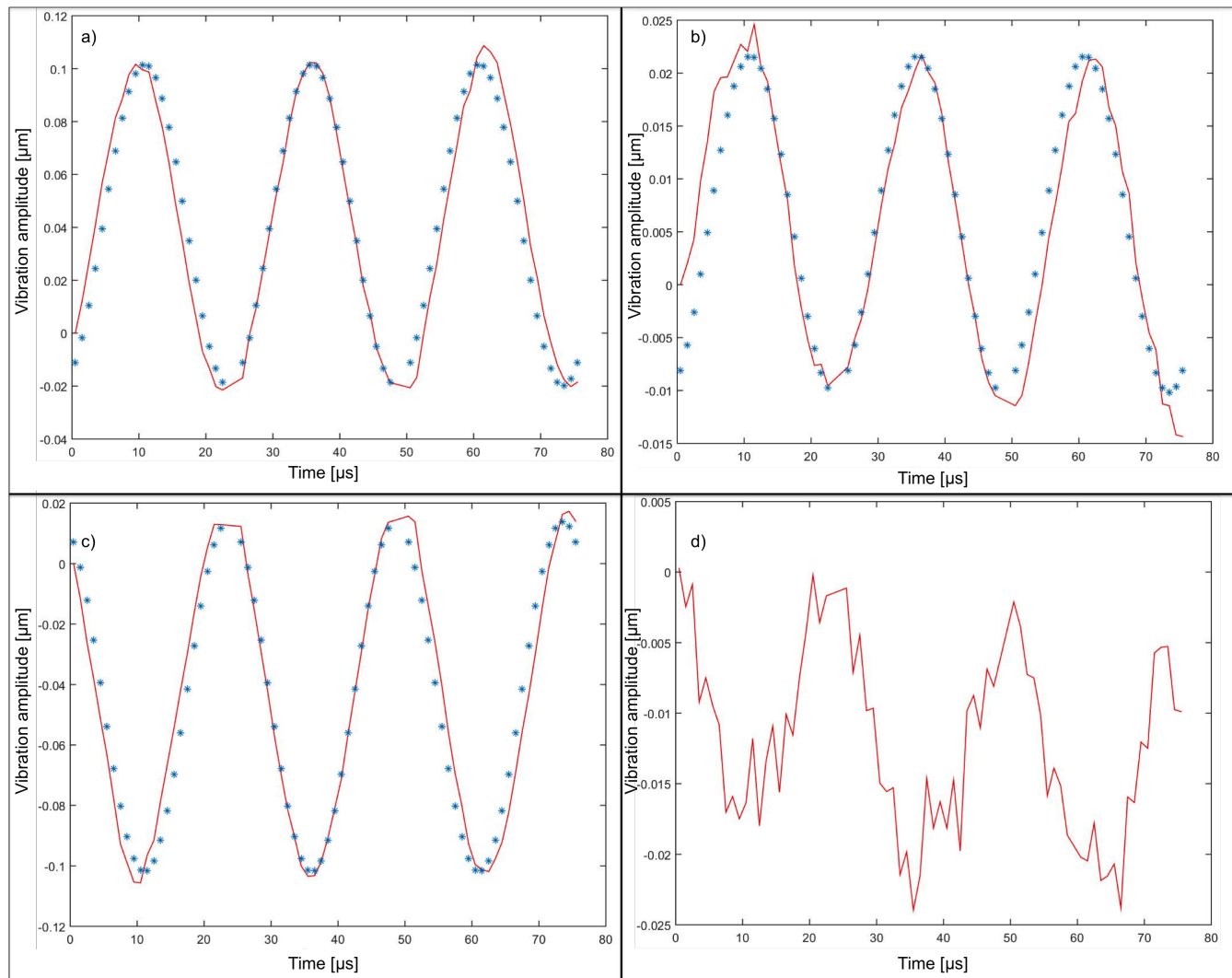

Figure 4.14: Amplitude analysis over time for the 4 different positions defined: a) 1 - Center; b) 3 - Intermediary position with weak amplitude of vibration; c) 4 - Intermediary position with strong amplitude of vibration; and d) 2 Edge.

position. Next, we extended the analysis for different measurement parameters: vibration amplitude, pulse duration and exposure time per image.

Initially, we are interested in analyzing the amplitude of vibration measured in function of the tension applied to the piezoelectric transducer. For this case, the duration of the stroboscopic pulse is $1 \mu \mathrm{s}$ at $40 \mathrm{kHz}$ and the exposure time of an image is $7500 \mu \mathrm{s}$. Hence, for the acquisition of one image, the sample is illuminated by the pulse 300 times, meaning the measurements are averaged over 300 periods at the instant of the pulse. To observe one complete period of the vibration $(25 \mu \mathrm{s})$, the acquisition time needs to be, therefore, $0.1875 \mathrm{~s}$. The results are presented in Figure 4.15. The four lines represent each position assumed for analysis, having the blue line for the results on the center of the transducer while the purple line shows the respective results for the edge. The intermediary positions are represented by the red and green line, for the weak and strong amplitude of vibration, respectively.

We can assess from the results on Figure 4.15 that the piezoelectric transducer moves in a relatively linear way in terms of the voltage. From the measurement at the edge, where no movement is expected, it is possible 


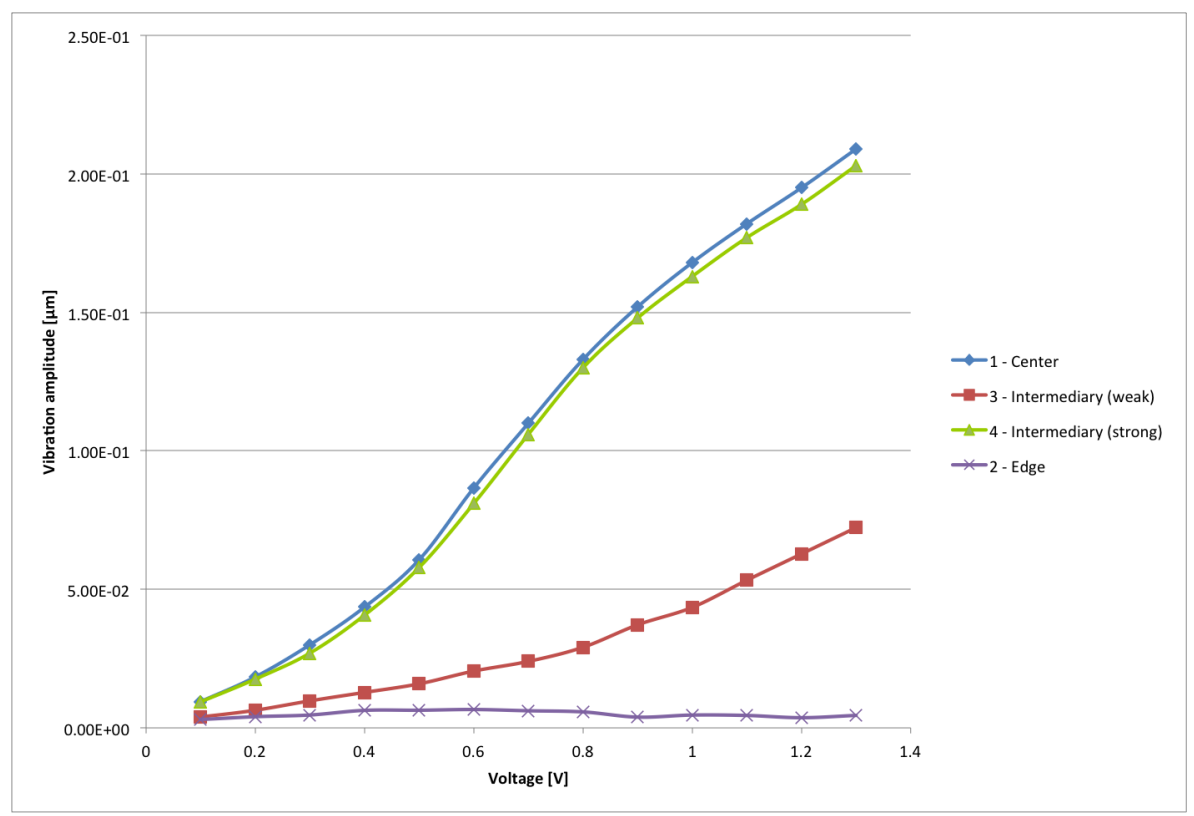

Figure 4.15: Amplitude of vibration analysis for different voltages applied to the transducer and at different positions of the transducer's surface. The blue line shows the results for the center of the transducer, the purple line for the edge, the red and green are the intermediary positions, for weak and strong amplitude of vibration, respectively.

to estimate the noise of the measurements. From these measurements, the noise varies between 3 and $6 \mathrm{~nm}$. It can be determined that this represents a mechanical noise.

To further asses the noise and better estimate it, a series of measurements with no vibration (zero voltage applied to the piezoelectric transducer) were run (Figure 4.16). We observed the noise over time at the center and the edge of the transducer in terms of the illumination time. The measurements were done according to the number of photons received by the sample at the moment of the acquisition of one image. We fixed the stroboscopic pulse duration at $1 \mu \mathrm{s}$ and we tested with different exposure time.

From Figure 4.16 we observe that the exposure time does not have a big influence on the noise of the measurement. However, it is necessary to assure that the measurement is not biased when acquired with lower level of illumination at the sample. To correctly assess this value, we compare in Figure 4.17 the noise when the piezoelectric is not running (Figure 4.17a) and when it is running (Figure 4.17b). Both measurements show of the amplitude in terms of the number of photons received by the sample when recording an image.

The piezoelectric transducer running with $1 \mathrm{~V}$ voltage and three different pulse duration were tested at the center of the transducer: $1 \mu s, 0.5 \mu s$ and 


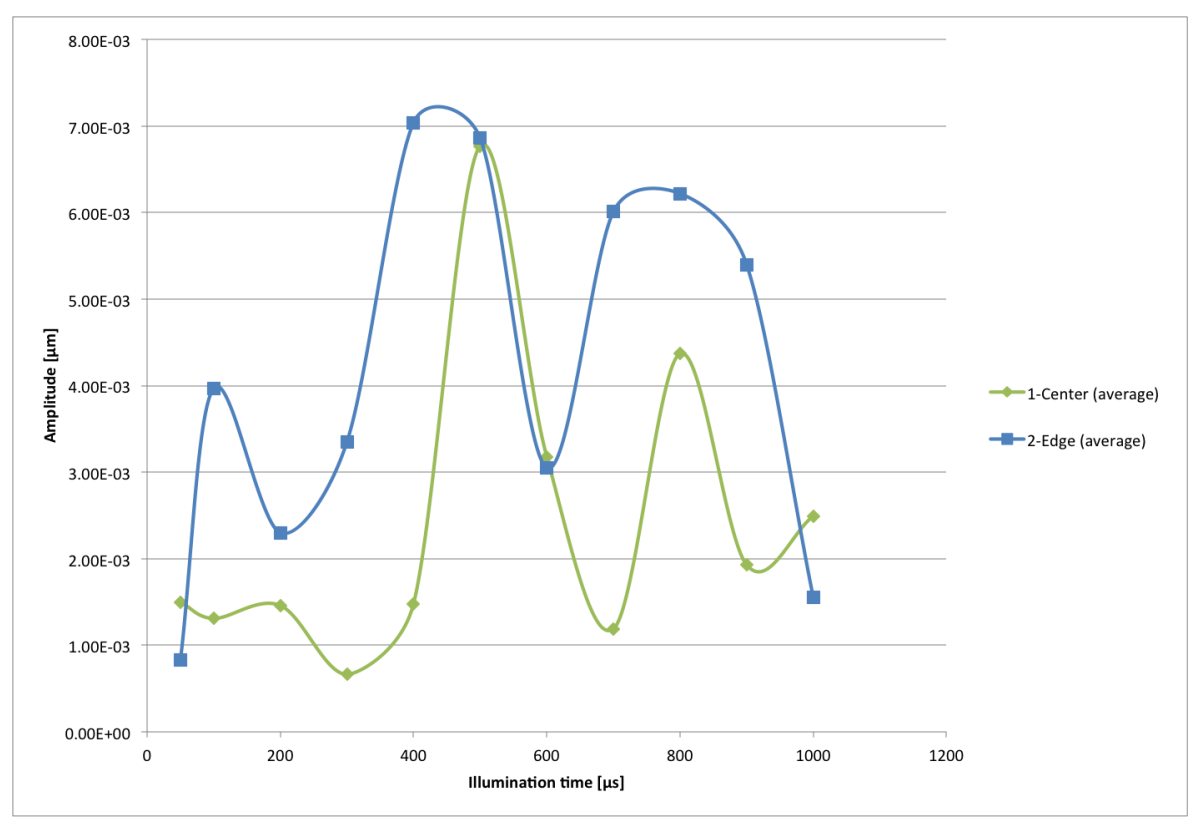

Figure 4.16: Noise analysis with zero voltage applied to the transducer (no vibration).

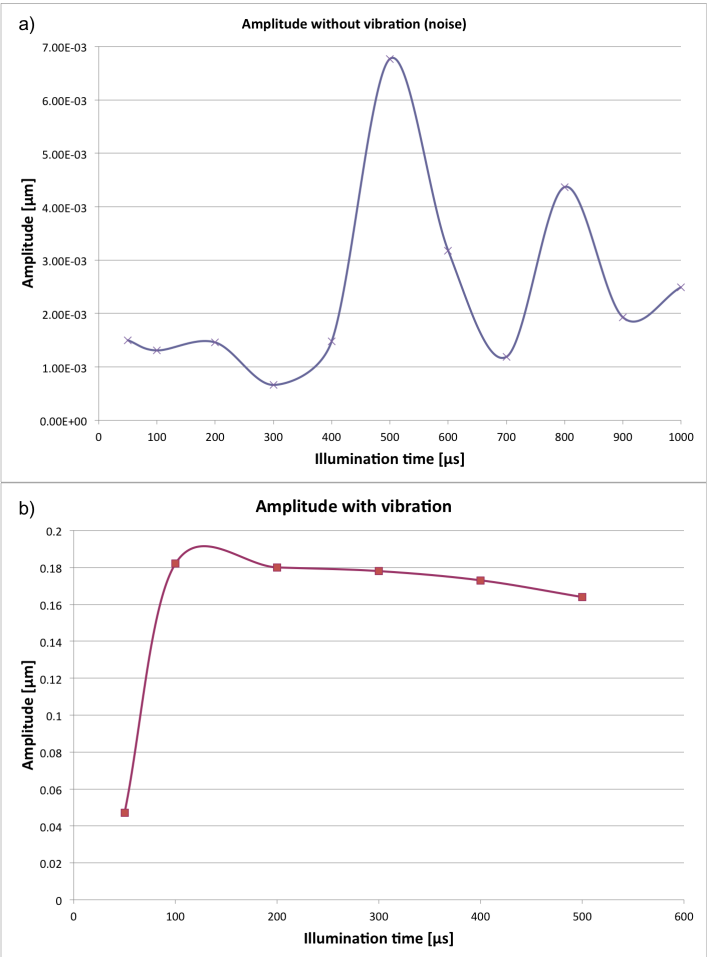

Figure 4.17: Comparison of amplitude with the transducer not running (a) and while it is running (b).

$2.5 \mu s$. The results obtained, illustrated in Figure 4.18, show that a illumination duration longer than $100 \mu \mathrm{s}$ with a laser power of $3.5 \mathrm{~mW}$ at the surface of the sample is needed for the different pulse duration tested. We can see from the results in Figure 4.18 that the pulse duration does not affect the vibration 
amplitude as the exposure time does.

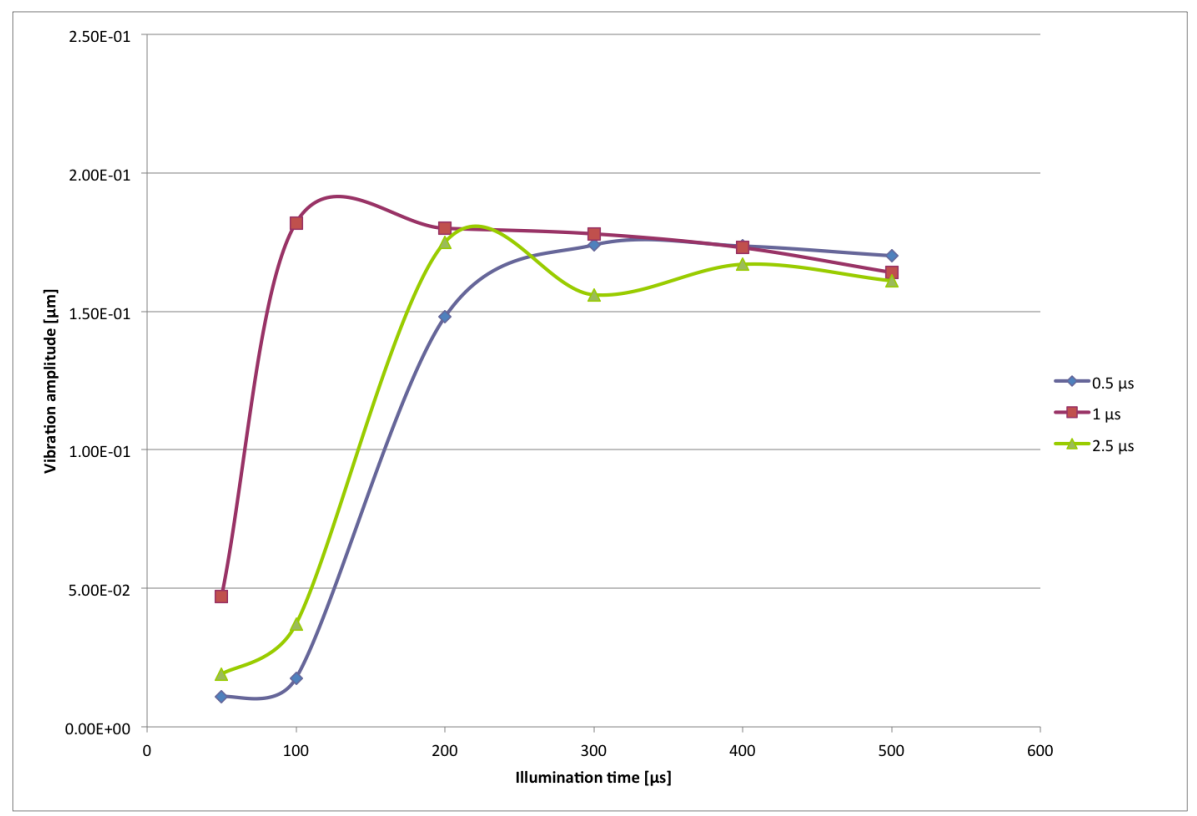

Figure 4.18: Vibration amplitude at the center of the transducer for 3 different pulse duration: $0.5 \mu s$ (blue line), $1 \mu s$ (red line) and $2.5 \mu s$ (green line).

This result shows that such system makes measurements in high frequencies complicated. It means we could not access the transient state, and to achieve such measurement, it would be necessary to reduce the pulse duration. To reduce the pulse duration in our current system, it would be required to rise the laser power or the exposure time for each image.

To properly assess the consistency of the measurements, a repeatability test was conducted. With a exposure time of $7500 \mu \mathrm{s}$, a stroboscopic pulse duration of $1 \mu \mathrm{s}$, where averaged over 300 periods, and the illumination time of the sample being $300 \mu s$, we repeated the same measurement 10 times. The results are presented in Figure 4.19 for all for positions at the surface of the piezoelectric transducer described earlier.

The repeatability results coherent with the noise previously estimated, which falls around $\pm 5-6 \mathrm{~nm}$. Further analyzing the repeatability results, we focus on one position, the center of the transducer (Figure 4.20). 


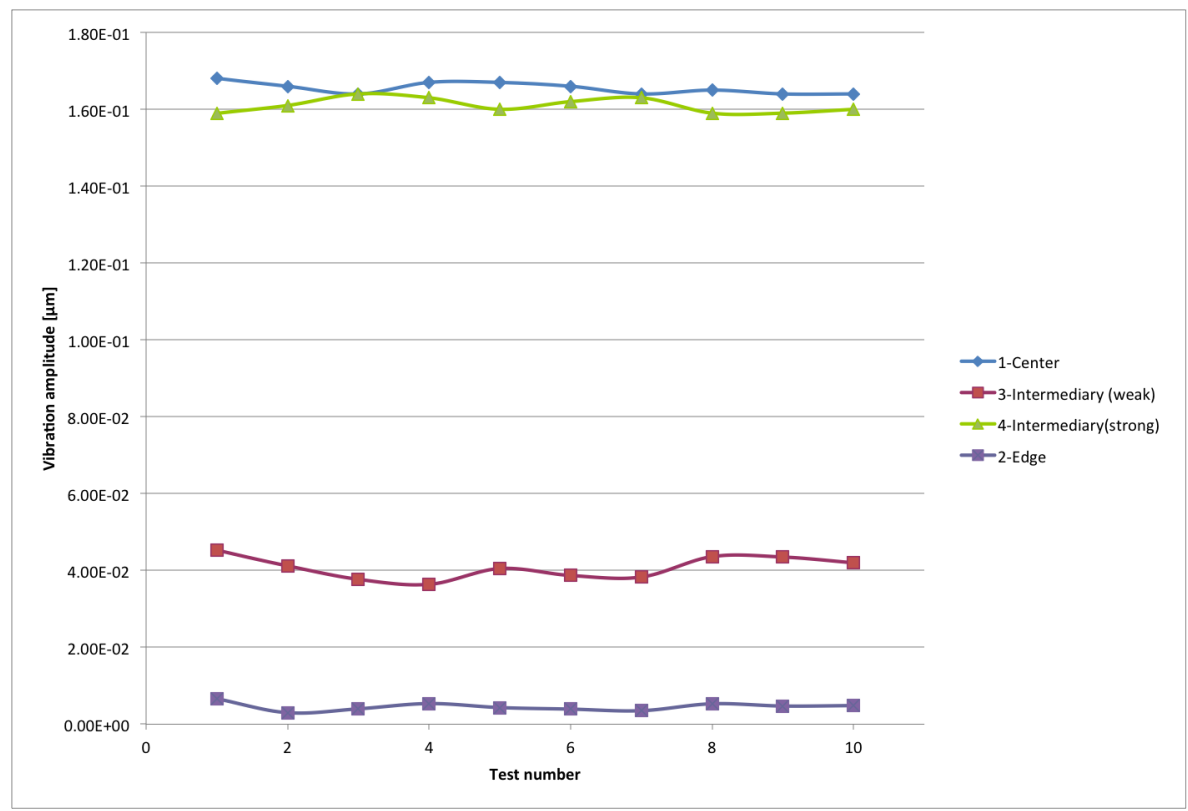

Figure 4.19: Repeatability test for different positions at the surface of the transducer. Blue line represents the center while purple are the results for the edge. The results for the intermediary positions are the red (weak) and green (strong) lines.

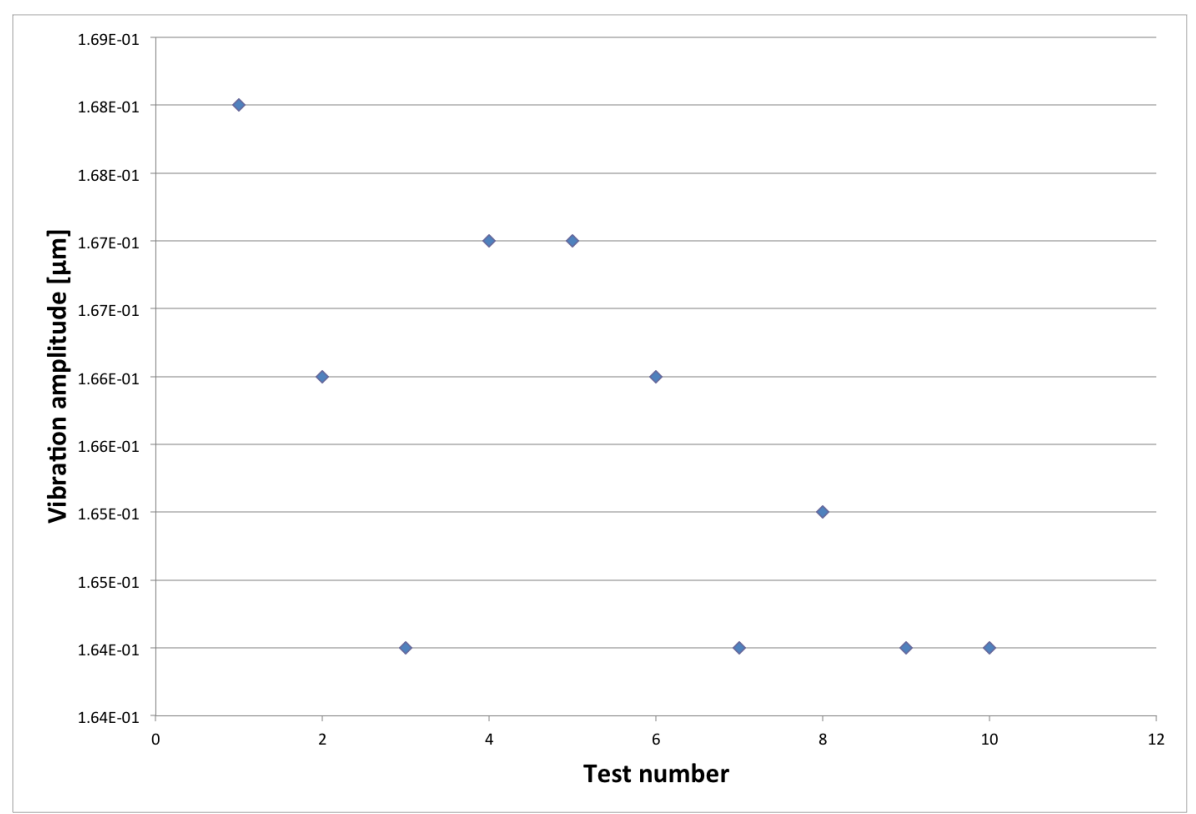

Figure 4.20: Analysis of the repeatability test only for the center of the transducer. 


\section{5 \\ Comparative study}

After presenting all the systems we assembled and tested experimentally, the results from the most promising systems were discussed in chapter 4: stroboscopic Mach-Zehnder and LOFI. In this chapter we discuss their most significant characteristics and compare their results to determine which system would perform better in the intended application of detecting acoustic signals.

\section{1}

\section{Parameters}

From the measurements presented and discussed earlier in this work, a few parameters were selected to compare and discuss both systems: noise, laser power, spatial resolution, temporal resolution and acquisition time.

\section{Noise}

We acquired noise measurements over time for both interferometric systems, as illustrated in Figure 5.1. The Stroboscopic Mach-Zehnder, a system that is spatially resolved, has a noise ranging from 3 to $6 \mathrm{~nm}$ (Figure 5.1a) averaged 300 times, as explained in chapter 4 . As for the LOFI, a system that is temporally resolved, has a noise 10 times lower, of the order of 5 angstroms averaged 10 times.

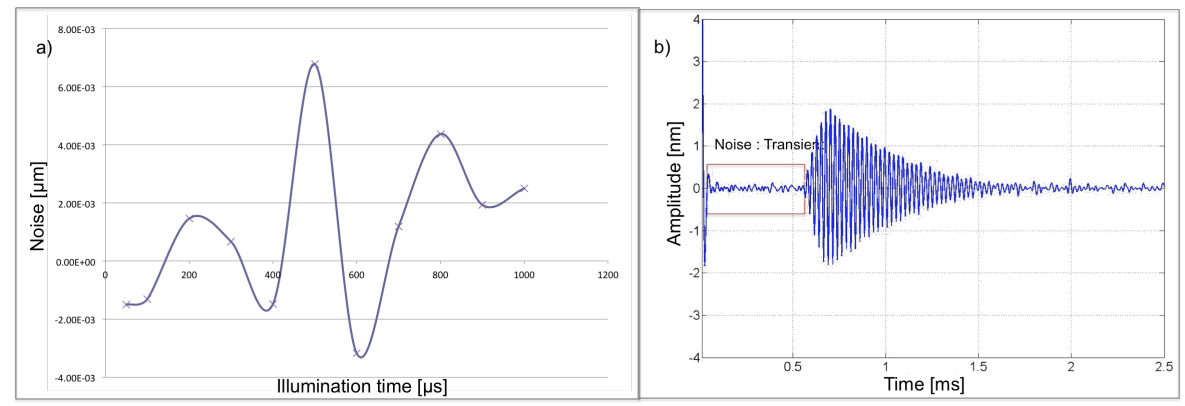

Figure 5.1: Noise measurements: (a) Stroboscopic Mach-Zehnder; and (b) LOFI.

It is important to note that the noise measured on both systems are dependent on the conditions used for such measurements, in particular, the 
laser power. For interferometric systems based on Mach-Zehnder that are spatially resolved, it is possible to achieve a noise level of 5 angstroms, despite not being visible in our results since we were limited by the mechanical noise. However, this implies a compromise with laser power arriving at the surface of the analyzed sample, meaning a much high power will be needed to remove part of the noise.

\section{Laser power}

Considering the same laser power for both systems, the stroboscopic Mach-Zehnder has a power of $4 \mathrm{~mW}$ at the sample, meaning $2 \mathrm{~mW} / \mathrm{cm}^{2}$ considering the area analyzed and the elements on our system. This value is less than the one defined by the medical standards for laser power on biological samples, which is $200 \mathrm{~mW} / \mathrm{cm}^{2}$ in the red part of the spectrum (wavelength ranging from 620 to $750 \mathrm{~nm}$ )(54). Additionally, this laser power is uniformly distributed at the sample. For the evaluated system, we would have around 15 nW per pixel and $75 \mathrm{nW}$ per speckle (considering 5 pixels per speckle).

For the LOFI, the laser power is not distributed uniformly over the analyzed area of the sample, since its illumination is point wise and needs to be scanned. The spot size of the LOFI's laser beam is $150 \mu \mathrm{m}$, which gives a power comparable to that of the stroboscopic Mach-Zehnder. That means the laser power of the LOFI at the sample of $4 \mathrm{~mW} / \mathrm{cm}^{2}$ (or $20 \mu \mathrm{m} / 0.5 \mathrm{~mm}^{2}$ ). The medical standard for laser power with a wavelength equals to $1064 \mathrm{~nm}$ (infrared) is $1 \mathrm{~W} / \mathrm{cm}^{2}$, much higher than what we're working with.

From a medical standards point of view, both systems comply with the acceptable values for laser power on biological samples with a good margin. This gives the techniques a margin to work on greater laser power on the sample in case it is needed.

\section{Spatial resolution}

The spatial resolution for the stroboscopic Mach-Zehnder depends, essentially, on a combination of the pixel size of the CCD sensor, the size of the speckle and the magnifying factor of the optical system. In our case, this means a spatial resolution of about $50 \mu \mathrm{m}$, since the pixel size of the CCD camera is $5.5 \mu \mathrm{m} \times 5.5 \mu \mathrm{m}$ and we have 4 pixels per speckle, plus the magnification effect of the system. One possible improvement by replacing the CCD sensor by one with a smaller pixel size. However, we cannot go lower than one single speckle, so we need to count, on average, the number of pixels of one speckle 
and consider the magnifying factor of the system to determine which size it corresponds on the sample.

Since the LOFI has a punctual acquisition, it needs a galvanometer scanner to quickly sweep the laser beam over the sample. For the studied system, the spatial resolution is of the hundreds of $\mu \mathrm{m}$. This value is depended on the optical elements of the system and to improve this means highly increase the price of the device.

\section{Temporal resolution}

Being a time based interferometric technique, the LOFI has a better temporal resolution compared to the stroboscopic Mach-Zehnder. Its temporal resolution is $10 \mu s, 1000 \mathrm{x}$ better than the Mach-Zehnder, which is of the order of $1 \mathrm{~ms}$.

In the LOFI method, the bandwidth is 10x larger than the stroboscopic Mach-Zehnder, being $1 \mathrm{MHz}$ compared to $100 \mathrm{kHz}$ on the Mach-Zehnder. The measuring area at the photon noise corresponds to a certain frequency range between the carrier and the Bessel peaks that represents a few MHz. With this, we can measure vibrations of the order of a few MHz.

For the Mach-Zehnder interferometer, we are limited by the duration of the stroboscopic pulse. This duration is also related to the sensitivity of the CCD sensor. With the current camera of our system and without using the gain, the temporal resolution is $1 \mathrm{~ms}$, which requires on a case of measuring at least 10 points for each period, for a frequency of $100 \mathrm{kHz}$. This is the reason why we did not run high frequency tests with this set up.

\section{Acquisition time}

Considering the fact that the laser beam needs to be scanned on the sample by the galvanometer scanner and the trigger associated with the procedure makes the acquisition time of 26 minutes for a $512 \times 512$ pixels video. This is certainly not ideal if considered for biomedical applications. This acquisition time is this long due to necessity to process each temporal acquisition before recording it because of a memory limitation. Clearly, this value can be improved by better acquisition cards.

As for the Mach-Zehnder, the acquisition time is around 2 seconds for a 512 x 512 pixels video, averaged 300 times for each image. This includes only the time required to trigger the equipment (the function generator that modulates both the $\mathrm{AOM}$ and the piezoelectric transducer and the $\mathrm{CCD}$ 
camera) and record the images in the computer. As it was mentioned before, this trigger and recording are controlled by a MATLAB program that could possibly be optimized.

The processing time, which is done by a separate MATLAB program, takes around 1 minute to complete and generate a video. Future works on this involves combining both acquisition and processing in one single, optimized, program. It is an advantage of the system to post process the measurements, separating the acquisition and processing steps.

\section{2}

\section{Discussion}

Table 5.1 summarizes the parameters discussed in the previous section. Regarding the noise and the laser power, the LOFI is superior to the stroboscopic Mach-Zehnder. That goes even considering the fact that the noise of the Mach-Zenhder is averaged 300 times.

The Mach-Zehnder system samples all points in time simultaneously while the LOFI is limited by the scan speed. No matter high the scan speed is, even if the LOFI could work with resonant scanners, it is always restricted to point by point measurement. For the moment, this point by point measurement has not been able to be parallelized. The Mach-Zehnder, despite having a slower sampling speed limited by the detector, can be truly simultaneous in the 2D space. This simultaneous detection can be advantageous for certain applications, especially where the phase difference in 2D space is important. Here, the stroboscopic Mach-Zehnder is intrinsically synchronized by the detector itself (this is true for many detectors but not rolling readout detectors which effectively scan in one dimension (59)).

The LOFI is a temporally resolved vibrometer that uses low optical power since it is amplified by an intrinsic gain (the resonance). Another advantage is the fact that it is a auto-aligned system compared to a slightly more complex alignment procedure with the Mach-Zehnder.

Another limitation of the stroboscopic system is the necessity of knowing the frequency of the vibration signal analyzed. The requirement of previously knowing the frequency of the vibration relies on the fact that the AOM needs to be modulated accordingly. This is a problem for conventional pulsed photoacoustic imaging, while in frequency domain photoacoustic imaging, this will not be an issue (60). It will be possible to know the vibration frequency in advance since it will be the same as the excitation frequency. However, to excite small biological samples, the vibration frequencies need to be of the order of 2.5 MHz. To be able to detect those frequencies with the Mach-Zehnder system, 
the pulse duration should be $40 \mathrm{~ns}$ and to have a minimum illumination time of $100 \mathrm{~ms}$, it needs to be averaged 2500 times. One possible way to go around changing the pulse duration is to rise the laser power, but keeping in my the medical standards for laser power. With these limitations, the LOFI is a more suited system for higher frequencies.

From the point of view of acquisition time and spatial resolution, the stroboscopic system is more advantageous if compared to the LOFI. It would be a better fit in real time applications, for example. For measurements of vibration waves, we can divide it in two scenarios. In an initial full field analysis of the sample, the stroboscopic Mach-Zehnder is ideal for a fast evaluation of the surface. Once the region of interest is assessed, the LOFI allows to access the transient state without being disturbed by external noise. Both systems are already viable for non destructive testing, but as for biomedical applications, high technology components are needed to push the systems to its limits.

Table 5.1: Comparison between LOFI and Mach-Zehnder.

\begin{tabular}{lcc}
\hline Parameters & LOFI & Stroboscopic MZ \\
\hline \hline $\begin{array}{c}\text { Acquisition time } \\
\text { Processing time }\end{array}$ & $6 \mathrm{~ms} /$ pixel $\dagger$ & $\begin{array}{c}2 \mathrm{~s}(512 \times 512 \text { pixels }) \ddagger \\
1 \mathrm{~min}(512 \times 512 \text { pixels })\end{array}$ \\
\hline Temporal Resolution & $10 \mu \mathrm{s}$ & $1 \mathrm{~ms}$ \\
\hline Spatial Resolution & $100 \mu \mathrm{m} \star$ & $50 \mu \mathrm{mo}$ \\
\hline Laser Power & $4 \mathrm{~mW} / \mathrm{cm}^{2}$ & $2 \mathrm{~mW} / \mathrm{cm}^{2}$ \\
\hline Noise & $<0.5 \mathrm{~nm}$ & $5 \mathrm{~nm}$ \\
\hline$\dagger 26$ min without average for $512 \times 512$ pixels \\
$\ddagger$ Averaged 300 times \\
$\star$ Depended of the optics \\
$\circ 4$ pixels per speckle plus the magnification \\
\hline \hline
\end{tabular}

It should be noted, however, that it is complicated to qualitatively compare these 2 systems. Both systems studied experimentally are limited by the elements used, which do not correspond to the most advanced components available. Faster galvanometer scanner, DAC with more memory or faster CCD sensors are a few examples of components that could improve each system performance. We base our discussion on the physics of each systems, keeping in mind that it could be improved by investing in more advanced technologies. 


\section{6}

\section{Conclusion and perspectives}

The present work analyzed and discussed the application of point scanning and full-field optical techniques in vibration detection with the aim on photoacoustic imaging systems. Among different full-field interferometric techniques studied, we chose to evaluate in further details the stroboscopic MachZehnder for the acquisition of ultrasound waves. To compare our results and also find a better suited system for the future intend application as detection of vibration signals in a photoacoustic system, a powerful point scanning technique, the LOFI, was evaluated and compared with the stroboscopic set-up.

The stroboscopic Mach-Zehnder is a full-field interferometric system, with fast acquisition and processing time, high spatial resolution with relatively low cost and low laser power on the surface of the sample.

The LOFI technique is an interesting tool to acquire high spatial resolution images in difficult conditions. The filtering of undesirable reflections in the LOFI signal allows measurements limited by the photon noise. The phase information allows us to measure vibrations with frequencies up to a few $\mathrm{MHz}$ with nanometric amplitudes. However, the acquisition speed is linked to the laser's response time, which in the case of microlasers, does not allow to reach video cadences. On the other hand, this response time could be greatly decreased by using laser diodes, with the detriment of the sensitivity of the technique. Therefore, as it is common place with imaging techniques, there is a compromise between sensitivity and acquisition speed.

The Mach-Zehnder system samples all points in time simultaneously while the LOFI is limited by the scan speed. No matter high the scan speed is, even if the LOFI could work with resonant scanners, it is always restricted to point by point measurement. The Mach-Zehnder, despite having a slower sampling speed limited by the detector, can be truly simultaneous in the 2D space. This simultaneous detection can be advantageous for certain applications, especially where the phase difference in 2D space is important. Here, the stroboscopic Mach-Zehnder is intrinsically synchronized by the detector itself.

The LOFI is a temporally resolved vibrometer that uses low optical power since it is amplified by an intrinsic gain, the resonance. Another advantage is 
the fact that it is a auto-aligned system compared to a slightly more complex alignment procedure with the Mach-Zehnder.

One limitation of the stroboscopic system is the necessity of knowing the frequency of the vibration signal analyzed. This is a problem for conventional pulsed photoacoustic imaging, while in frequency domain photoacoustic imaging, this will not be an issue (60). It would be possible to know the vibration frequency in advance since it will be the same as the excitation frequency. However, to excite small biological samples, the vibration frequencies need to be of the order of $2.5 \mathrm{MHz}$. To be able to detect those frequencies with the Mach-Zehnder system, the pulse duration should be $40 \mathrm{~ns}$ and to have a minimum illumination time of $100 \mathrm{~ms}$, it needs to be averaged 2500 times. One possible way to avoid changing the pulse duration is to rise the laser power, but keeping in my the medical standards for laser power. With these limitations, the LOFI is a more suited system for higher frequencies.

From the point of view of acquisition time and spatial resolution, the stroboscopic system is more advantageous if compared to the LOFI. It would be a better fit in real time applications, for example. For measurements of vibration waves, we can divide it in two scenarios. In an initial full field analysis of the sample, the stroboscopic Mach-Zehnder is ideal for a fast evaluation of the surface. Once the region of interest is assessed, the LOFI allows to access the transient state without being disturbed by external noise. Both systems are already viable for non destructive testing, but as for biomedical applications, high technology components are needed to push current design to even be on par with other techniques on the field.

The conclusion from the analysis and comparison of these two system is that they are complementary to one another. An ideal concept would be to use the two systems for biomedical applications, where, first, a full field image would be acquired for a rapid evaluation of the sample. This would serve to determine the area of interest to be further analyzed. We would, then, use the LOFI on this smaller section of the sample to access the transient state, which is only limited by the photon noise.

Further analysis into the LOFI, focusing on its non-linearity, it was demonstrated how quantitative vibration measurements can be extracted from the non-linear dynamics of a laser submitted to frequency shifted optical feedback. We have studied the non-linear dynamical coupling between the modulation at the carrier frequency and the modulation of the vibration sidebands at the acoustic frequency.

For small vibrations, it was shown how the non-linear amplification of the vibration spectrum by the laser dynamics is related to the strength of the 
optical feedback and, therefore, of the amplitude of the carrier. It also shown how this amplification can be compensated to obtain vibration measurements without bias in the nanometer range at relatively high frequency, of the order of several hundred of $\mathrm{kHz}$. The theoretical predictions and the numerical simulations are in good agreement with the experimental data for both transient and harmonic vibrations.

Encouraged by the preliminary results on the sensitivity and performances of the non-linear LOFI device, it will be tested for the optical detection with a photon-noise sensitivity of acoustics waves induced by a photoacoustic effect. For the full-field technique, one set-up that has shown good results is a heterodyne synchronous detection $(61,62)$. Another approach is an optical heterodyne system based on all fiber acousto-optic frequency shifter, that is capable of measuring micro-vibrations (63).

One important point must be made regarding the qualitatively comparison of these two systems. Both systems studied experimentally are limited by the elements used on their respective set up at the laboratory, which do not correspond to the most advanced components available. With more advanced technologies as faster galvanometer scanner, DAC with more memory or faster CCD sensors, the performance of each system could be significantly improved. The discussion presented, therefore, bases itself on the physics of each system, keeping in mind that it could be improved by their different components. 


\section{Bibliography}

[1] BISHOP, R. E. D.. Vibration. Cambridge University Press, 2nd edition, 1979.

[2] EWINS, D. J.. Modal Testing: Theory, Practice, and Application. Research Studies Press, 2nd edition, 2000.

[3] BRUEL; KJAER. Frequency Analysis. Naerum, Denmark : Bruel amd Kjaer, 1987.

[4] BRUEL; KJAER. Measuring vibration, 1982.

[5] ROYLANCE, L. M.; ANGELL, J. B.. A batch-fabricated silicon accelerometer. IEEE Transactions on Electron Devices, 26(12):1911-1917, 1979.

[6] CASTELLINI, P.; MARTARELLI, M. ; TOMASINI, E.. Laser doppler vibrometry: Development of advanced solutions answering to technology's needs. Mechanical Systems and Signal Processing, 20(6):1265 1285, 2006. Special Issue: Laser Doppler Vibrometry.

[7] CUMMINS, H. Z.; KNABLE, N. ; YEH, Y.. Observation of diffusion broadening of rayleigh scattered light. Phys. Rev. Lett., 12:150-153, Feb 1964.

[8] YEH, Y.; CUMMINS, H.. Localized fluid flow measurements with an he-ne laser spectrometer. Applied Physics Letters, 4(10):176-178, 1964.

[9] BENDICK, P.. A laser doppler velocimeter to measure instantaneous velocity profiles. In: PROCEEDINGS OF THE FIRST SYMPOSIUM ON FLOW, ITS MEASUREMENT AND CONTROL IN SCIENCE AND INDUSTRY, p. 1033-1036, 1971.

[10] RIVA, C.; ROSS, B. ; BENEDEK, G. B.. Laser doppler measurements of blood flow in capillary tubes and retinal arteries. Investigative Ophthalmology \& Visual Science, 11(11):936, 1972. 
[11] BRIERS, J. D.. Laser doppler, speckle and related techniques for blood perfusion mapping and imaging. Physiological Measurement, 22(4):R35, 2001.

[12] MIKAMI, O.; FUJIKAWA, C.. 3-beam laser doppler velocimeter for 3-d velocity measurement. In: 2016 IEEE 6TH INTERNATIONAL CONFERENCE ON PHOTONICS (ICP), p. 1-3, March 2016.

[13] FERCHER, A. F.; DREXLER, W.; HITZENBERGER, C. K. ; LASSER, T.. Optical coherence tomography - principles and applications. Reports on Progress in Physics, 66(2):239, 2003.

[14] SIEGMAN, A. E.. The antenna properties of optical heterodyne receivers. Appl. Opt., 5(10):1588-1594, Oct 1966.

[15] LONGO, R.; VANLANDUIT, S.; VANHERZEELE, J. ; GUILLAUME, P.. A method for crack sizing using laser doppler vibrometer measurements of surface acoustic waves. Ultrasonics, 50(1):76-80, 2010.

[16] BEARD, P.. Biomedical Photoacoustic Imaging: a review. Interface Focus, 1(June):602-631, 2011.

[17] WANG, L. V.; HU, S.. Photoacoustic Tomography: In Vivo Imaging from Organelles to Organs. Science, 335(6075):1458-1462, 2012.

[18] BURGHOLZER, P.; GRUN, H. ; SONNLEITNER, A.. Photoacoustic tomography: Sounding out fluorescent proteins. Nat Photon, 3(7):378-379, 072009.

[19] ZACKRISSON, S.; VEN, S. M. W. Y. V. D. ; GAMBHIR, S. S.. Light In and Sound Out : Emerging Translational Strategies for Photoacoustic Imaging. Cancer Research, 74(4):979-1004, 2014.

[20] XU, M.; WANG, L. V.. Photoacoustic imaging in biomedicine. Review of Scientific Instruments, 77(4):041101, 2006.

[21] HU, S.; WANG, L. V.. Photoacoustic imaging and characterization of the microvasculature. Journal of Biomedical Optics, 15(1):011101, 2010.

[22] PAGE, D. A.. Handbook of Optical Metrology: Principles and Applications, chapter Interferometry, p. 191-218. CRC Press, 2009.

[23] GOODMAN, J. W.. Some fundamental properties of speckle. Journal of the Optical Society of America, 66(11):1145, 1976. 
[24] ENNOS, A.. $\{$ IV $\}$ speckle interferometry. volumen 16 de Progress in Optics, p. 233 - 288. Elsevier, 1978.

[25] CREATH, K.. Phase-measurement interferometry techniques. volumen 26 de Progress in Optics, p. 349 - 393. Elsevier, 1988.

[26] GHIGLIA, D. C.; PRITT, M. D.. Two-dimensional phase unwrapping: theory, algorithms, and software. J. Wiley \& Sons, New York, 1998.

[27] OTSUKA, K.. Effects of external perturbations on lindp4o12lasers. IEEE Journal of Quantum Electronics, 15(7):655-663, Jul 1979.

[28] HUGON, O.; PAUN, I.; RICARD, C.; VAN DER SANDEN, B.; LACOT, E.; JACQUIN, O. ; WITOMSKI, A.. Cell imaging by coherent backscattering microscopy using frequency-shifted optical feedback in a microchip laser. Ultramicroscopy, 108(6):523 - 528, 2008.

[29] LACOT, E.; DAY, R. ; STOECKEL, F.. Laser optical feedback tomography. Opt. Lett., 24(11):744-746, Jun 1999.

[30] LACOT, E.; DAY, R. ; STOECKEL, F.. Coherent laser detection by frequency-shifted optical feedback. Phys. Rev. A, 64:043815, Sep 2001.

[31] LACOT, E.; JACQUIN, O.; ROUSSELY, G.; HUGON, O. ; DE CHATELLUS, H. G.. Comparative study of autodyne and heterodyne laser interferometry for imaging. J. Opt. Soc. Am. A, 27(11):2450-2458, Nov 2010.

[32] SIEGMAN, E.. Lasers, chapter Laser spiking and mode competition, p. 955-971. California, University Science Books, Mill Valley, 1986.

[33] DAY, R.. Une nouvelle technique d'imagerie laser basée sur la réinjection décalée en fréquence. Laser Optical Feedback Imaging ( LOFI ). PhD thesis, Université Joseph Fourier - Grenoble I, 2000.

[34] JACQUIN, O.; LACOT, E.; GLASTRE, W.; HUGON, O. ; DE CHATELLUS, H. G.. Experimental comparison of autodyne and heterodyne laser interferometry using an nd:yvo4 microchip laser. J. Opt. Soc. Am. A, 28(8):1741-1746, Aug 2011.

[35] HUGON, O.; JOUD, F.; LACOT, E.; JACQUIN, O. ; DE CHATELLUS, H. G.. Coherent microscopy by laser optical feedback imaging (lofi) technique. Ultramicroscopy, 111(11):1557 - 1563, 2011. 
[36] GLASTRE, W. G.. Plenoptic imaging through complex media using synthetic aperture imaging. Theses, Université de Grenoble, Sept. 2013.

[37] OTSUKA, K.; ABE, K.; KO, J.-Y. ; LIM, T.-S.. Real-time nanometervibration measurement with a self-mixing microchip solid-state laser. Opt. Lett., 27(15):1339-1341, Aug 2002.

[38] HUNG, Y. Y.. Shearography : a new optical method for strain measurement and nondestructive testing. Optical Engineering, 21(3):391395, 1982.

[39] LEENDERTZ, J. A.; BUTTERS, J. N.. An image-shearing specklepattern interferometer for measuring bending moments. Journal of Physics E: Scientific Instruments, 6(11):1107, 1973.

[40] HUNG, Y. Y.. A Speckle-shearing interferometer: a tool for measuring derivatives of surface displacements. Optics Communications, 11(2):132-135, 1974.

[41] HUNG, Y.. Digital shearography versus TV-holography for nondestructive evaluation. Optics and Lasers in Engineering, 26(4-5):421436, mar 1997.

[42] HUNG, Y.; HO, H.. Shearography: An optical measurement technique and applications. Materials Science and Engineering: R: Reports, 49(3):61-87, apr 2005.

[43] FOCKE, O.; HILDEBRAND, A.; VON KOPYLOW, C. ; CALOMFIRESCU, M.. Inspection of Lamb Waves in Carbon Fiber Composites using Shearographic Interferometry. Proc. of SPIE, 6934:693403-12, mar 2008.

[44] PEDRINI, G.; ZOU, Y.-L. ; TIZIANI, H. J.. Quantitative evaluation of digital shearing interferogram using the spatial carrier method. Pure and Applied Optics: Journal of the European Optical Society Part A, 5:313-321, 1996.

[45] NG, T.. Shear measurement in digital speckle shearing interferometry using digital correlation. Optics Communications, 115(34):241-244, 1995.

[46] HUNG, Y. Y.; LIANG, C. Y.. Image-shearing camera for direct measurement of surface strains. Applied optics, 18(7):1046-51, apr 1979. 
[47] STEINCHEN, W.; KUPFER, G. ; MACKEL, P.. Vibration analysis by digital shearography. The Journal of the Acoustical Society of America, 108(5):2622, 2000.

[48] FRANCIS, D.; TATAM, R. P. ; GROVES, R. M.. Shearography technology and applications: a review. Measurement Science and Technology, 21(10):102001, oct 2010.

[49] XIE, X.; LI, X.; CHEN, X. ; YANG, L.. Review of recent developments of spatial phase-shift digital shearography. In: Quan, C.; Qian, K.; Asundi, A. ; Chau, F. S., editors, INTERNATIONAL CONFERENCE ON EXPERIMENTAL MECHANICS, volumen 9302, p. 93020E, mar 2015.

[50] ERF, R.. Holographic Nondestructive Testing. Elsevier, 1974.

[51] CUCHE, E.; BEVILACQUA, F. ; DEPEURSINGE, C.. Digital holography for quantitative phase-contrast imaging. Optics letters, 24(5):291293, 1999.

[52] CUCHE, E.; MARQUET, P. ; DEPEURSINGE, C.. Spatial filtering for zero-order and twin-image elimination in digital off-axis holography. Applied optics, 39(23):4070-4075, 2000.

[53] DE GROOT, P.. Stroboscopic white-light interference microscopy. Appl. Opt., 45(23):5840-5844, Aug 2006.

[54] LI, C.; WANG, L. V.. Photoacoustic tomography and sensing in biomedicine. Physics in medicine and biology, 54(19):R59-R97, 102009.

[55] THORLABS. Thorlabs resolution test targets. https://www . thorlabs.com/newgrouppage9. cfm?objectgroup_id=4338\&pn=R1DS1P, 2016. Accessed: 2016-09-06.

[56] TAKEDA, M.. Spatial-carrier fringe-pattern analysis and its applications to precision interferometry and profilometry: An overview. Industrial Metrology, 1(2):79-99, jun 1990.

[57] TAKEDA, M.; INA, H. ; KOBAYASHI, S.. Fourier-transform method of fringe-pattern analysis for computer-based topography and interferometry. Journal of the Optical Society of America, 72(1):156, 1982.

[58] GIRARDEAU, V.; GOLONI, C.; JACQUIN, O.; HUGON, O.; INGLEBERT, M. ; LACOT, E.. Nonlinear laser dynamics induced by frequency 
shifted optical feedback: application to vibration measurements. Appl. Opt., 55(34):9638-9647, Dec 2016.

[59] FORSSÉN, P.-E.; RINGABY, E.. Rectifying rolling shutter video from hand-held devices. In: CVPR10, SAN FRANSISCO, USA, JUNE 13-18, 2010, p. 507-514. IEEE Computer Society, 2010.

[60] LeBOUllueC, P.; LIU, H. ; YUAN, B.. A cost-efficient frequencydomain photoacoustic imaging system. American Journal of Physics, 81(9):712-717, 2013.

[61] GROSS, M.; JOUD, F.; ATLAN, M.; TAILLARD, P.-A.; VERPILLAT, F. ; VERRIER, N.. Vibration motions studied by heterodyne holography. In: DIGITAL HOLOGRAPHY AND THREE-DIMENSIONAL IMAGING, p. DTh3A.5. Optical Society of America, 2013.

[62] VERRIER, N.; ATLAN, M.. Absolute measurement of small amplitude vibrations by time-averaged heterodyne holography with a dual local oscillator. Opt. Lett., 38(5):739-741, Mar 2013.

[63] ZHANG, W.; GAO, W.; HUANG, L.; MAO, D.; JIANG, B.; GAO, F.; YANG, D.; ZHANG, G.; XU, J. ; ZHAO, J.. Optical heterodyne micro-vibration measurement based on all-fiber acousto-optic frequency shifter. Opt. Express, 23(13):17576-17583, Jun 2015.

[64] LANG, R.; KOBAYASHI, K.. External optical feedback effects on semiconductor injection laser properties. IEEE Journal of Quantum Electronics, 16:347-355, Mar. 1980.

[65] ERNEUX, T.; KOVANIS, V. ; GAVRIELIDES, A.. Nonlinear dynamics of an injected quantum cascade laser. Phys. Rev. E, 88:032907, Sep 2013.

[66] TAIMRE, T.; NIKOLIĆ, M.; BERTLING, K.; LIM, Y. L.; BOSCH, T. ; RAKIĆ, A. D.. Laser feedback interferometry: a tutorial on the self-mixing effect for coherent sensing. Adv. Opt. Photon., 7(3):570-631, Sep 2015.

[67] OTSUKA, K.. Self-mixing thin-slice solid-state laser metrology. Sensors, 11(2):2195-2245, 2011.

[68] OKAMOTO, S.; TAKEDA, H. ; KANNARI, F.. Ultrahighly sensitive laser-doppler velocity meter with a diode-pumped nd:yvo4 microchip laser. Review of Scientific Instruments, 66(5):3116-3120, 1995. 
[69] KAWAI, R.; ASAKAWA, Y. ; OTSUKA, K.. Ultrahigh-sensitivity selfmixing laser doppler velocimetry with laser-diode-pumped microchip lindp4o12 lasers. IEEE PHOTONICS TECHNOLOGY LETTERS, 11(6):706-708, JUN 1999.

[70] SUDO, S.; OHTOMO, T.; TAKAHASHI, Y.; OISHI, T. ; OTSUKA, K.. Determination of velocity of self-mobile phytoplankton using a selfmixing thin-slice solid-state laser. APPLIED OPTICS, 48(20):40494055, JUL 102009.

[71] LACOT, E.; HUGON, O.. Phase-sensitive laser detection by frequency shifted optical feedback. Phys. Rev. A, 70:053824, Nov 2004.

[72] MUZET, V.; LACOT, E.; HUGON, O. ; GUILLARD, Y.. Experimental comparison of shearography and laser optical feedback imaging for crack detection in concrete structures. In: Osten, W.; Gorecki, C. ; Novak, E. L., editors, OPTICAL MEASUREMENT SYSTEMS FOR INDUSTRIAL INSPECTION IV, volumen 5856, p. 793-799, June 2005.

[73] PETERMANN, K.. Laser diode modulation and noise, 1988.

[74] KOLOBOV, M. I.; DAVIDOVICH, L.; GIACOBINO, E. ; FABRE, C.. Role of pumping statistics and dynamics of atomic polarization in quantum fluctuations of laser sources. Phys. Rev. A, 47:1431-1446, Feb 1993.

[75] TAN, Y.; XU, C.; ZHANG, S. ; ZHANG, S.. Power spectral characteristic of a microchip nd:yag laser subjected to frequency-shifted optical feedback. Laser Physics Letters, 10(2):025001, 2013.

[76] TAN, Y.; ZHANG, S.; ZHANG, S.; ZHANG, Y. ; LIU, N.. Response of microchip solid state laser to external frequency-shifted feedback and its applications. Scientific Reports, 3, 2013.

[77] LACOT, E.; HOUCHMANDZADEH, B.; GIRARDEAU, V.; HUGON, O. ; JACQUIN, O.. Non linear modification of the laser noise power spectrum induced by frequency-shifted optical feedback. Submitted to Phys. Rev. A, 2016.

[78] SCHWARTZ, I. B.; ERNEUX, T.. Subharmonic hysteresis and period doubling bifurcations for a periodically driven laser. SIAM Journal on Applied Mathematics, 54(4):1083-1100, 1994. 
[79] WITOMSKI, A.; LACOT, E.; HUGON, O. ; ERNEUX, T.. Parametric amplification of frequency-shifted optical feedback. Phys. Rev. A, 72:023801, Aug 2005.

[80] PHILLIPS, M.; GONG, H.; FERGUSON, A. ; HANNA, D.. Optical chaos and hysteresis in a laser-diode pumped nd doped fibre laser. Optics Communications, 61(3):215 - 218, 1987.

[81] CELET, J. C.; DANGOISSE, D.; GLORIEUX, P.; LYTHE, G. ; ERNEUX, T.. Slowly passing through resonance strongly depends on noise. Phys. Rev. Lett., 81:975-978, Aug 1998. 


\section{A \\ Appendix: Non-linear laser dynamics induced by frequency shifted optical feedback}

Laser properties (power, polarization, coherence, dynamical behavior) can be significantly affected and modified by optical feedback $(64,65)$ which allows for the realization of non-conventional sensors. One potential application is Laser Feedback Interferometry (LFI), where the steady-state intensity of a laser is modified by coherent optical feedback from an external surface. With this phase sensitive technique, the signal depends on the reflectivity, distance and motion of the target (66). However, when the amount of reinjected light is very small, the interference contrast occurring inside the laser cavity is drastically reduced. To overcome this problem, one solution is to use the dynamical properties of the laser which can be several orders of magnitude more sensitive to optical feedback than the laser steady-state properties.

Since the pioneering work of K. Otsuka on self-mixing modulation effect in a class-B laser (27), the dynamical sensitivity of lasers to frequency shifted optical feedback has been used in metrology (67), for example in self mixing Laser Doppler Velocimetry (LDV) $(68,69,70)$ and in Laser Optical Feedback Imaging (LOFI) $(29,35,36)$. Compared to conventional optical heterodyne detection, frequency shifted optical feedback allows for higher (several order of magnitude) intensity modulation contrast (30, 31, 34).

For weak optical feedback, the laser dynamics is linear and the maximum of the modulation is reached when the shift frequency is resonant with the laser relaxation oscillation frequency. In this condition, an optical feedback level as low as $-170 \mathrm{~dB}\left(10^{17}\right.$ times weaker than the laser intra-cavity power) has been detected (68).

Phase measurements (such as profilometry or vibrometry) with low noise needs to increase the amount of optical feedback reinjected inside the laser cavity $(37,71,72)$. But, for strong optical feedback, the laser dynamics becomes non-linear, making it difficult to obtain quantitative measurements.

In this appendix, we start by recalling the equations governing the dynamics of a laser with a frequency-shifted optical reinjection back-scattered from a vibrating target and the signal processing to extract vibration measurements from the laser dynamics. We follow by presenting the numerical 
Appendix A. Appendix: Non-linear laser dynamics induced by frequency shifted optical feedback

simulations vibration signals (harmonic and transient) extracted from the laser dynamic in both the linear and nonlinear regimes. These numerical simulations show that the gain of the laser dynamics needs to be compensated to extract, without bias, the vibration amplitude.

A bifurcation analysis is made to obtain an asymptotic solution for the gain of the laser dynamics. Our calculations show that the carrier and the vibration signal are coupled through the non-linear laser dynamics. We demonstrate that the nonlinear amplification of the vibration spectrum is related to the amplitude of the carrier signal. Therefore, our calculation confirms that to obtain quantitative vibration measurements, the non-linear gain must be taken into account in the signal processing. The theoretical predictions presented, confirmed by the numerical simulations, are in good agreement with the experimental data discussed in chapter 4 .

\section{A.1}

\section{Laser with frequency-shifted optical feedback}

The use of a LOFI set up for our experiments was motivated by the following reasons:

o the LOFI interferometer is always self-aligned because the laser simultaneously fulfills the functions of source (photons-emitter) and photodetector (photons-receptor);

o the LOFI detection is photon noise limited (even with a low power laser) in a frequency range located near the relaxation oscillation frequency of the laser $(30,31,34)$.

In this appendix we present the equations governing the dynamics of a laser with a frequency-shifted optical reinjection, or feedback, back scattered from a vibrating target.

Using a bifurcation analysis, we show how the carrier and the vibration signal are coupled through the non-linear laser dynamics and we demonstrate that the non-linear amplification of the vibration spectrum is related to the amplitude of the carrier signal. Therefore, to obtain quantitative vibration measurements, this non-linear gain needs to be compensated through signal processing.

By using the non-linear gain previously determined, we show typical examples of vibration signals (harmonic and transient) extracted from the non-linear laser dynamic. The theoretical predictions, confirmed by numerical simulations, are in good agreement with the experimental data presented and discussed in chapter 4 . 
Appendix A. Appendix: Non-linear laser dynamics induced by frequency shifted optical feedback

\section{A.1.1}

\section{Basic equations for vibrometry}

For weak optical feedback (effective power reflectivity $R_{e} \ll 1$ ) and a short round trip time delay ( $\tau_{e} \ll 1 / F_{0}$ ), the dynamical behavior of a laser with a frequency shifted $\left(F_{0}\right)$ optical feedback can be described by the following set of differential equations (30):

$$
\begin{aligned}
\frac{d I}{d t} & =B N I-\gamma_{c} I-2 \gamma_{c} \sqrt{R_{e}} I \cos \left(\Omega_{0} t-\Phi_{e}(t)\right)+F_{1}(t), \\
\frac{d N}{d t} & =\gamma_{1}\left(N_{0}-N\right)-B N I \\
\left\langle F_{1}(t)\right\rangle & =0 \\
\left\langle F_{1}(t) F_{1}(t-\tau)\right\rangle & =2 \gamma_{c}\langle I\rangle \delta(\tau)
\end{aligned}
$$

where $\mathrm{N}$ is the population inversion (atom unit), $\gamma_{1} N_{0}$ is the pumping rate, $\mathrm{B}$ is related to Einstein coefficient (laser cross section), I is the number of photons in the laser cavity, $\gamma_{1}$ is the decay rate of the population inversion, $\gamma_{c}$ is the laser cavity decay rate and $\gamma_{1} N_{0}$ is the pumping rate. $R_{e}$ is the effective reflectivity coefficient of the sample, taking into account the diffusing aspect of the object imaged as well as the recovery between the wave emitted by the laser and the wave injected into the laser. Regarding the noise, the laser quantum fluctuations are described by the Langevin noise function $F_{1}(t)$, with a zero mean value and a white noise type correlation function $(73,74)$.

In $\mathrm{A}-1 \mathrm{a}$, the cosine function expresses the coherent interaction (i.e. the beat at the shift frequency: $\Omega_{0}=2 \pi F_{0}$ ) between the lasing and the feedback electric field. The optical reinjection backscattered from a vibrating target is characterized by the effective power reflectivity $R_{e}$ and the time dependent optical phase shift $\Phi_{e}(t)=\frac{2 \pi}{\lambda_{c}} 2 d_{e}(t)$ induced by the distance $d_{e}(t)$ between the laser the vibrating target, and $\lambda_{c}$ is the laser's wavelength.

In the absence of optical feedback $\left(R_{e}=0\right)$, the laser steady-state is given by the mean values of the population inversion and of the laser intensity:

$$
\begin{aligned}
N_{S} & =\frac{\gamma_{c}}{B}, \\
I_{S} & =I_{s a t}[\eta-1],
\end{aligned}
$$

where $\eta=N_{0} / N_{S}$ is the quantum efficiency and $I_{\text {sat }}=\gamma_{1} / B$ is related to the saturation intensity of the laser transition. Also for $R_{e}=0$, the intrinsic dynamics of a class-B laser $\left(\gamma_{c} \gg \eta \gamma_{1}\right)$ is characterized by damped relaxation oscillations of the laser output power with a relaxation angular frequency $\Omega_{R}=\sqrt{\gamma_{1} \gamma_{c}(\eta-1)}$ and a damping rate $\Gamma_{R}=\eta \gamma_{1} / 2$. Experimentally, this 
Appendix A. Appendix: Non-linear laser dynamics induced by frequency shifted optical feedback

transient dynamics is constantly excited by the laser quantum noise described by the Langevin force $F_{1}(t)$.

In the presence of optical feedback $\left(R_{e} \neq 0\right)$, the laser intrinsic dynamics are modified and the extent of changes in the laser dynamical properties depends on the feedback conditions. We call strong feedback the case where the modulation frequency is nearly resonant $\left(\Omega_{0}=\Omega_{R}\right)$ and where the amount of optical feedback is high enough to induce non-linear dynamical behaviors in the laser output power modulation. The strong feedback situation is also characterized by a strong modification of the RF signal power spectrum of the laser $(75,76)$. In particular, the relaxation oscillations frequency can decrease, jointly with a modification of the shape of the noise power spectrum of the laser in the radio frequency (RF) range (77).

In contrast, the weak feedback regime corresponds to the situation where the modulation frequency is far away from the resonance $\left(\left|\Omega_{0}-\Omega_{R}\right| \gg 0\right)$ and where the amount of optical feedback is small enough to induce only linear dynamical behavior in the laser output power modulation. In this situation, the noise power spectrum of the laser in RF range, with and without optical feedback, is unchanged. The RF noise power spectrum is an image of the modulation transfer function (gain) of the laser dynamics (77). The knowledge of this gain is of paramount importance to extract without bias the vibration measurements from the laser dynamics of a reinjected laser, as will be discussed in the following section.

\section{A.1.2}

\section{Signal processing for vibrometry}

The aim of the present paper is to explain how it is possible to extract the time dependence of the phase shift of the optical feedback namely $\Phi_{e}(t)$ (and consequently $d_{e}(t)$ ) from the laser dynamics, in both the weak and the strong feedback regimes.

For a laser with frequency shifted optical feedback, the output power modulation of the laser is given by the convolution product between the input modulation (i.e. the coherent beating at the carrier frequency) and the impulse response of the laser dynamics. The impulse response of the laser dynamics is the inverse Fourier transform $\left(\mathcal{F}^{-1}\right)$ of the gain of the laser dynamics $G\left(\Omega, R_{e}, \Omega_{0}\right)$ :

$$
\Delta I(t)=I(t)-I_{S} \propto \sqrt{R_{e}}\left[\mathcal{F}^{-1}\left\{G\left(\Omega, R_{e}, \Omega_{0}\right)\right\} \otimes \exp \left[i\left(\Omega_{0} t+\Phi_{e}(t)\right)+c c\right]\right]
$$

In Equation A-4, the gain of the laser dynamics can depend of the feedback condition, specially in the strong feedback regime. To extract the 
Appendix A. Appendix: Non-linear laser dynamics induced by frequency shifted optical feedback

temporal evolution of the phase $\Phi_{e}(t)$ induced by the vibration, the different steps of the signal processing are given by the set of the following equations:

$$
\begin{array}{r}
\mathcal{F}\{\Delta I(t)\} \propto \sqrt{R_{e}}\left\{G\left(\Omega, R_{e}, \Omega_{0}\right) \times\left[\mathcal{F}\left\{\exp \left(i \Phi_{e}(t)\right)\right\} \otimes \delta\left(\Omega-\Omega_{0}\right)\right]+\right. \\
\left.G^{*}\left(\Omega, R_{e}, \Omega_{0}\right) \times\left[\mathcal{F}\left\{\exp \left(-i \Phi_{e}(t)\right)\right\} \otimes \delta\left(\Omega+\Omega_{0}\right)\right]\right\} \\
\mathcal{F}_{\Delta \Omega_{0}}\{\Delta I(t)\} \propto \sqrt{R_{e}}\left\{G\left(\Omega, R_{e}, \Omega_{0}\right) \times\left[\mathcal{F}\left\{\exp \left(i \Phi_{e}(t)\right)\right\} \otimes \delta\left(\Omega-\Omega_{0}\right)\right]\right\} \\
\frac{\mathcal{F}_{\Delta \Omega_{0}} \Delta I(t)}{G\left(\Omega, R_{e}, \Omega_{0}\right)} \propto \sqrt{R_{e}}\left\{G\left(\Omega, R_{e}, \Omega_{0}\right) \times\left[\mathcal{F}\left\{\exp \left(i \Phi_{e}(t)\right)\right\} \otimes \delta\left(\Omega-\Omega_{0}\right)\right]\right\} \\
\left.\mathcal{F}^{-1}\left\{\frac{\mathcal{F}_{\Delta \Omega_{0}} \Delta I(t)}{G\left(\Omega, R_{e}, \Omega_{0}\right)}\right\} \propto \sqrt{R_{e}} \exp \left(i \Phi_{e}(t)\right) \exp \left(i \Omega_{0} t\right)\right) \\
\mathcal{F}^{-1}\left\{\frac{\left.\left.\mathcal{F}_{\Delta \Omega_{0} \Delta I(t)}\right\} \exp \left(-i \Omega_{0} t\right)\right) \propto \sqrt{R_{e}} \exp \left(i \Phi_{e}(t)\right)}{G\left(\Omega, R_{e}, \Omega_{0}\right)}\right\} \\
\left.\arg \left\{\mathcal{F}^{-1}\left\{\frac{\mathcal{F}_{\Delta \Omega_{0}} \Delta I(t)}{G\left(\Omega, R_{e}, \Omega_{0}\right)}\right\} \exp \left(-i \Omega_{0} t\right)\right)\right\}=\Phi_{e}(t)
\end{array}
$$

In Equation A-5, the Fourier Transform of the time evolution of the laser output power is calculated. Then, the Fourier spectrum is filtered using a bandpass filter with a central frequency $\Omega_{0}$ and a bandwidth $\Delta \Omega_{0}$ in Equation A-6. The bandwidth is adapted to the frequency range of the vibration spectrum. Following to Equation A-7, the distortion of the vibration spectrum induced by the laser dynamics, the amplification gain, is compensated. We return to the temporal space in Equation A-8 and the signal is demodulated [Equation A-9 and finally, in Equation A-10, the temporal evolution of the phase is extracted from the complex signal.

In this signal processing, it can be noticed that the multiplication, by any real constant number, of the input signal (the laser output power modulation), has no impact on the extraction of output signal, which is a phase signal. So, the gain can be multiplied by any real constant. Therefore, for the experimental measurement of vibrations presented in section 4.1, the absolute knowledge of the amplitude of the gain was not necessary. This means that only the complex frequency shape is needed when acquiring experimental measurements. This is due to the fact that, for vibration measurements, only the relative ratio between the amplitudes of the spectral components is important.

\section{A.1.3}

\section{Compensation of the gain of the laser dynamics}

To demonstrate how different kinds of vibrations can be extracted from the laser dynamics, we have numerically solved the set of differential equations given by Equation A-1. 
Appendix A. Appendix: Non-linear laser dynamics induced by frequency shifted optical feedback

\section{A.1.3.1 \\ Numerical harmonics vibrations}

Figure A.1 shows examples of the calculations of a harmonic vibration:

$$
\Phi_{e}(t)=\Phi_{a} \sin \left(\Omega_{a} t\right)
$$

with an acoustic angular frequency $\Omega_{a}=\Omega_{R} / 3.5$ and with a small vibration amplitude $\Phi_{a}=2 \pi / 50$.

The calculations were made for a given frequency shift $\left(\Omega_{0}=1.1 \Omega_{R}\right)$ and for two optical feedback reflectivities $\left(R_{e}=10^{-9}\right.$ and $\left.R_{e}=10^{-7}\right)$. When the feedback is weak $\left(R_{e}=10^{-} 9\right)$, it can be noted from plot a) in Figure A.1 that the gain adjusted on the noise power spectrum exhibits only one resonance (linear). On the other hand, when the feedback is strong $\left(R_{e}=10^{-7}\right)$, the gain, which becomes non-linear, exhibits two resonances symmetrically located on both side of the carrier frequency (plot b) (77).

The middle row (plots $\mathrm{c}$ and $\mathrm{d}$ ) show a comparison between the exact vibration motion and the vibration obtained after the signal processing, without the gain compensation. This means that the step given by Equation A7 of the signal processing was not done. Neither the amplitude nor the phase of the detected vibration on these plots present the desired results. In the weak feedback case, the carrier signal, which is near the resonance, is amplified more than the vibration sidebands and thus the vibration amplitude is lower than the real one. In the strong feedback case, the opposite situation occurs. The vibration sidebands, which are near the resonances, are amplified above the carrier signal, and therefore, the detected vibration amplitude is larger than the real one. In both cases, a $\pi$ phase-shift was induced by the resonance.

The last row of Figure A.1 (plots e and f) show that the accurate vibration is recovered if the complex gain compensation is done in the signal processing. These results clearly show that the gain compensation (linear or non-linear) is necessary to accurate quantitative vibration measurements of amplitude and phase from the reinjected laser dynamics.

The comparison of plots e) and f) also shows that the SNR increases when the amount of optical feedback increases. This effect is mainly due to the increase of all the vibration components relative to the noise baseline. So, even for a strong optical feedback, where the laser dynamics becomes non-linear, the compensation of the non-linear amplification allows to restore accurate vibration measurement with an improved SNR. Therefore, with the non-linear gain compensation the detection of very small vibration amplitudes is possible with a reinjected laser. 
Power spectrum (dB)
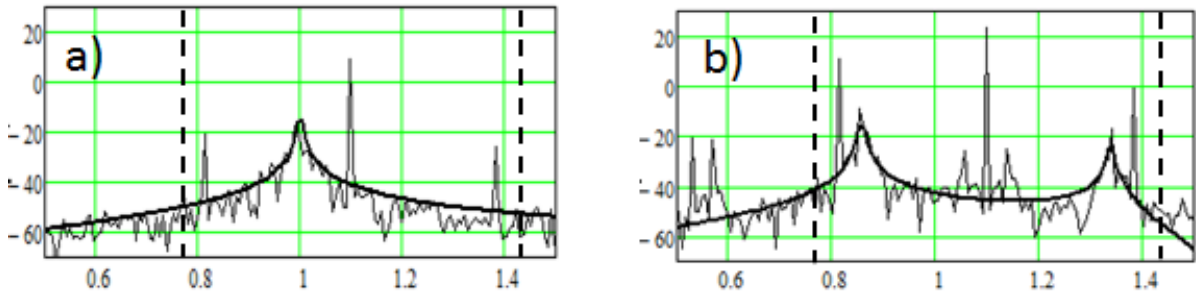

Normalized frequency: $F / F_{R}$

\section{Reconstructed phase(rad) without gain compensation}
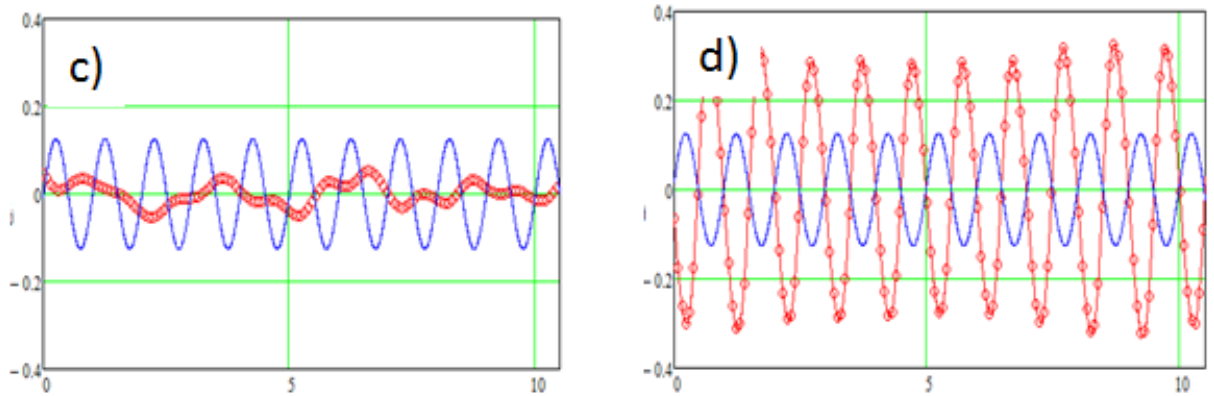

Normalized time: $\mathrm{txF}_{\mathrm{a}}$

\section{Reconstructed phase(rad) with gain compensation}
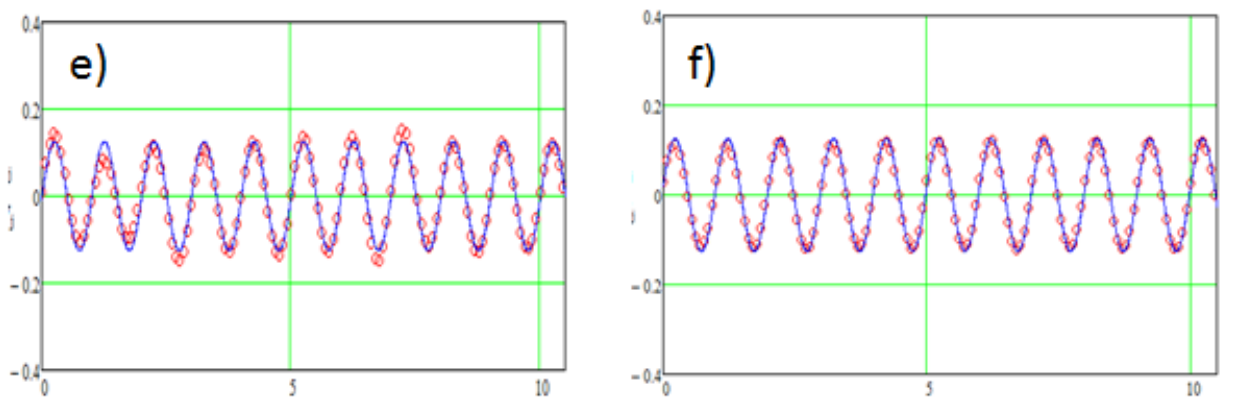

Normalized time: $\mathrm{txF}_{\mathrm{a}}$

Figure A.1: Calculations of a harmonic vibration $\left(\Omega_{a}=\Omega_{R} / 3.5\right)$ with a small amplitude of vibration $\left(\Phi_{a}=2 \pi / 50\right)$. Plots a), c) and e) correspond to a weak optical feedback $\left(R_{e}=10^{-9}\right)$ while plots b), d) and f) correspond to a strong optical feedback $\left(R_{e}=10^{-7}\right)$. The top row (a and b) shows the laser power spectrum where the resonance gain (bold line) is adjusted on the noise spectrum. The calculations are made with and a bandpass filter $\Delta \Omega_{0}=\Omega_{R} / 1.5$ (see dashed vertical lines). The middle row (c, d) shows a comparison between the exact vibration motion (solid line) and the vibration obtained after the signal processing (line of circles), but without the gain compensation. The bottom row $(e, f)$ shows the same comparison when the gain compensation has been applied. Laser parameters: $\eta=1.2, \frac{\gamma_{1}}{\gamma_{c}}=10^{-5}, \frac{F_{R}}{\gamma_{c}}=2.251 \times 10^{-4}$ (58). 
Appendix A. Appendix: Non-linear laser dynamics induced by frequency shifted optical feedback

\section{A.1.3.2}

\section{Numerical transient vibrations}

To verify that our signal processing can be applied to any kind of vibrations, we have numerically simulated transient vibrations:

$$
\Phi_{e}(t)=\frac{\Phi_{a}}{1+\left(\frac{t-\tau}{\tau_{a}}\right)^{2}} .
$$

With a half time width $\tau_{a} \approx 1.8 / F_{R}$ (i.e. a broad spectral bandwidth $\left.\Delta \Omega a \approx \Omega_{R} / 1.8\right)$ and a time delay $\tau=16.4 \tau_{a}$. Figure A.2 shows examples of the extraction of an impulse vibrations for 3 different values of the amplitudes $\left(\Phi_{a}=2 \pi / 5, \Phi_{a}=2 \pi / 50\right.$ and $\left.\Phi_{a}=2 \pi / 500\right)$. The numerical simulation have been made for a given frequency shift $\Omega_{0}=1.1 \Omega_{R}$ and for strong feedback reflectivity $R_{e}=10^{-7}$.

For the first analysis of the 3 images on the right column, the non-linear gains used for the gain compensation in the signal processing is always the same, also being identical to the one used in Figure A.1 for the calculation of harmonic vibration (left column). This is due to the fact that the feedback conditions are the same and, consequently, the gain of the laser dynamics is the same for all these vibration calculations. The plots on the right column of Figure A.2 (b, d and f) show that the impulse vibration with the accurate amplitude and the accurate time delay can be extracted from the non-linear laser dynamics.

The impulse shape is recovered regardless of the vibration amplitude, even when the vibration amplitude is large (see Figure A.2 plots a and b) and when the RF power spectrum is strongly perturbed by non-linear dynamical effects of higher order.

Finally, on Figure A.2f, an amplitude of $d_{a}=\frac{\lambda}{1000}=1 \pm 0.2 \mathrm{~nm}$ is detected, showing the high potential of this measurement method for the detection of small vibration amplitudes at relatively high frequencies. It must be noted that the SNR of the order of 5 has been obtained with a filtering process (see Equation A-6) adapted to the width of the vibration spectrum $\left(\Delta \Omega_{0}=\Omega_{R} / 1.5 \approx \Delta \Omega_{a}\right)$.

For a narrower bandwidth $\left(\Delta \Omega_{0}<\Delta \Omega_{a}\right)$ the noise (i.e. the fluctuation at the base of the impulse vibration) is lower, but the vibration spectrum is truncated. Consequently, the amplitude and width of the reconstructed vibration pulse is not correct. Conversely, if the bandwidth is wider $\left(\Delta \Omega_{0}>\Delta \Omega_{a}\right)$, the noise increases without any significant improvement of the reconstructed pulse shape.

In Figure A.3, the transient vibration is again a short impulse vibration 
Power spectrum $(\mathrm{dB})$
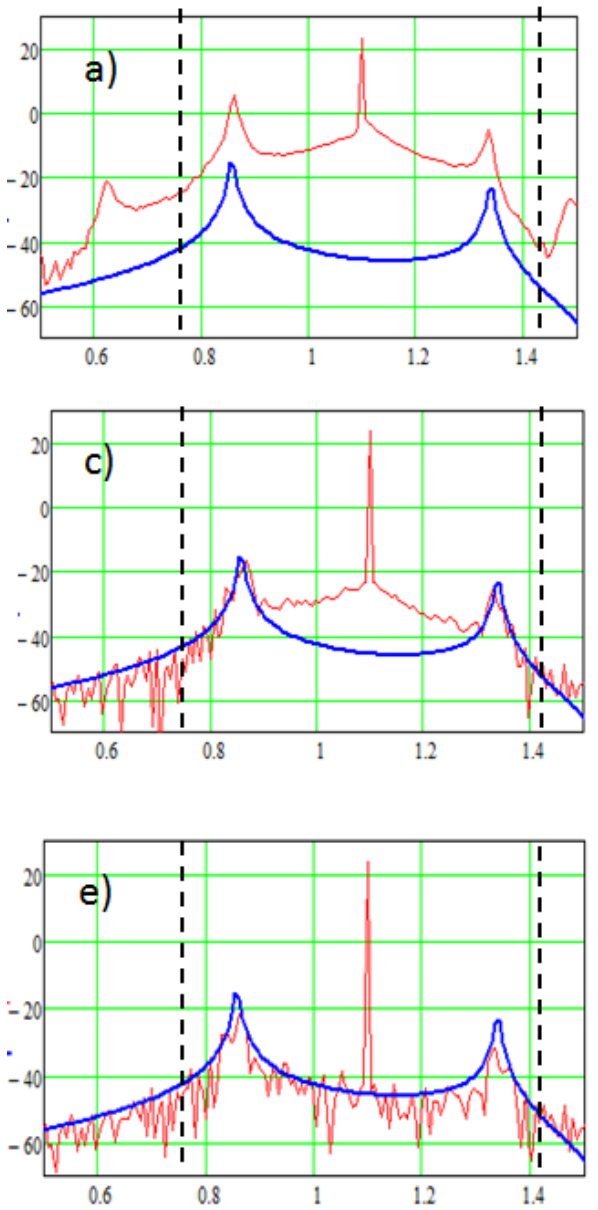

Normalized frequency: $F / F_{R}$
Transient vibration(nm)
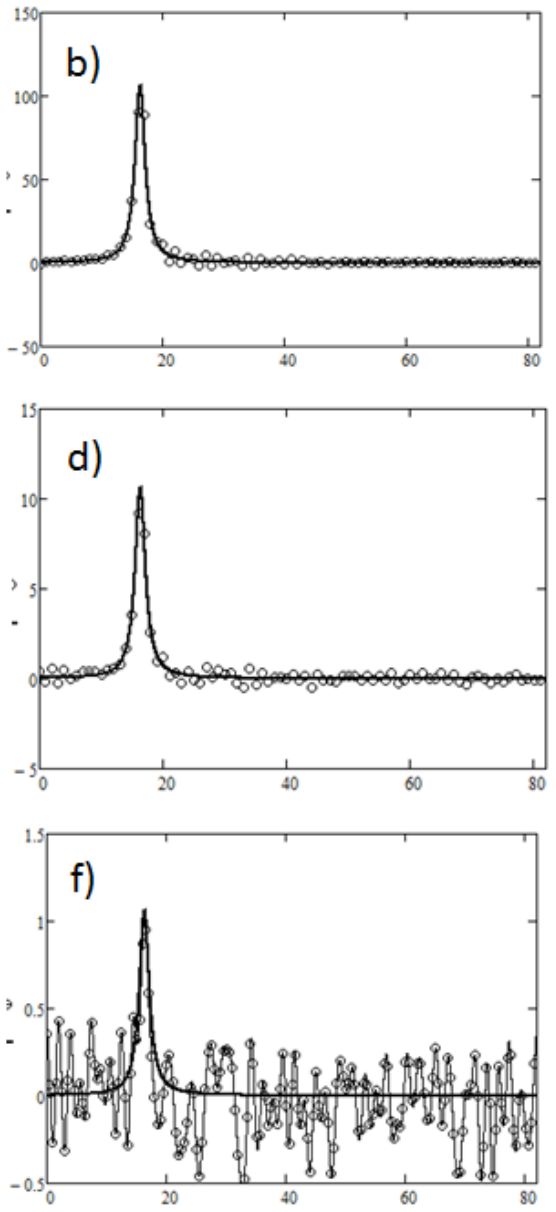

Normalized time: $\mathrm{t} / \tau_{\mathrm{a}}$

Figure A.2: Calculations of a transient vibration with a full time width. Top row $(\mathrm{a}, \mathrm{b})$ : large amplitude of vibration $\left(\Phi_{a}=2 \pi / 5\right)$. Middle row (c, d): small amplitude of vibration $\left(\Phi_{a}=2 \pi / 50\right)$. Bottom row (e,f): very small amplitude of vibration $\left(\Phi_{a}=2 \pi / 500\right)$. The left column (a, c, e) shows the RF power spectra, where the bold lines show the shape of the non-linear gain used for the signal processing. The right column (b, d, f) shows a comparison between the exact vibration motion (solid line) and the vibration obtained after the signal processing (circles). All the calculations are made with the same feedback conditions $\left(R_{e}=10^{-7}\right.$ and $\left.\omega_{0}=1.1 \omega_{R}\right)$ and the same bandpass filter $\Delta \Omega_{0}=\Omega_{R} / 1.5$ (corresponding to the dashed vertical lines). (58)

with a wide spectral bandwidth $\left(\Delta \Omega_{0}=\Omega_{R} / 1.8\right)$ and with a small vibration amplitude $\left(\Phi_{a}=2 \pi / 50\right)$, but now the calculations have been made for a strong feedback reflectivity $\left(R_{e}=10^{-7}\right)$ and for 3 different values of the carrier frequency $\left(\Omega_{0} / \Omega_{R}=1.3, \Omega_{0} / \Omega_{R}=1.1\right.$, and $\left.\Omega_{0} / \Omega_{R}=1.01\right)$.

The left column shows how the non-linear gain is modified when the carrier frequency approaches the intrinsic laser relaxation frequency (i.e. when $\left.\frac{\Omega_{0}}{\Omega_{R}} \rightarrow 1\right)$. As already mentioned, the modification of the non-linear gain is 

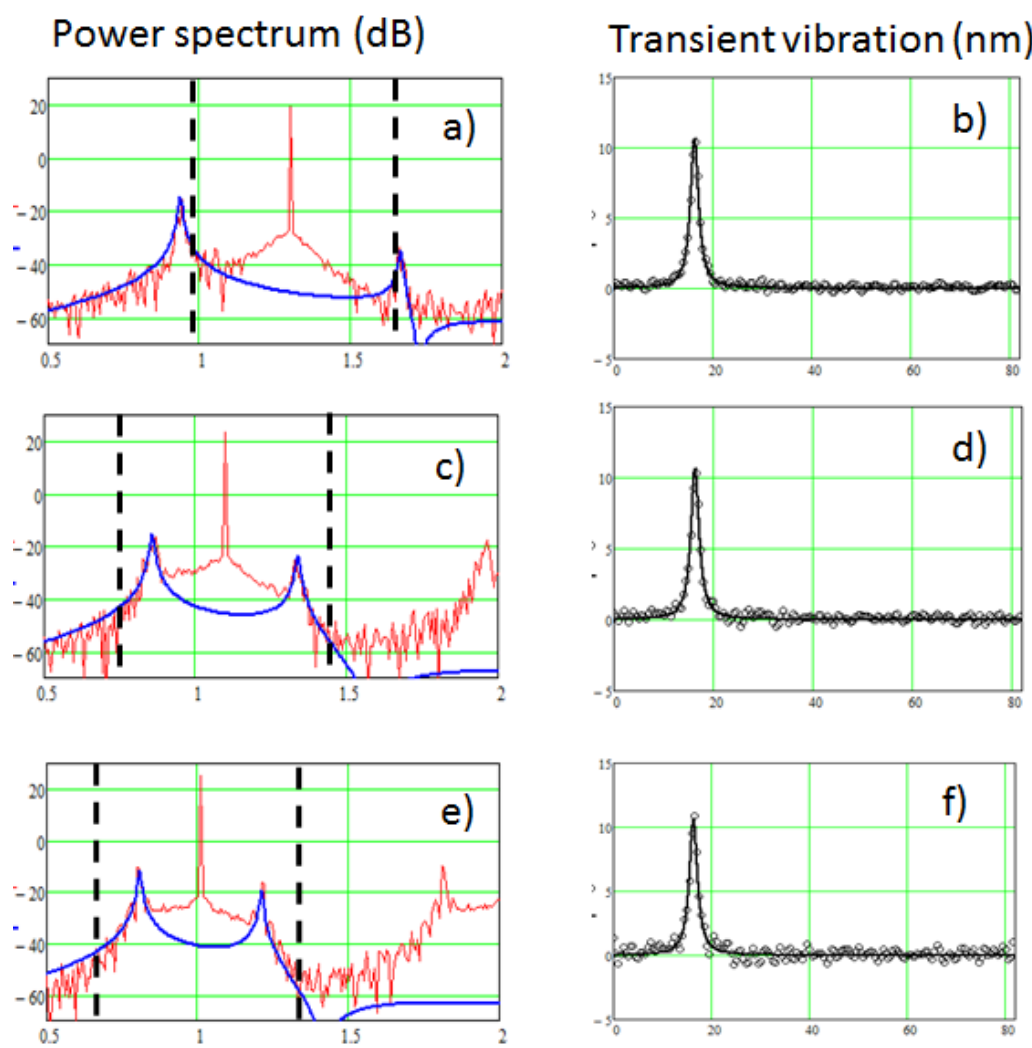

Normalized frequency: $F / F_{R}$

Normalized time: $\mathrm{t} / \tau_{\mathrm{a}}$

Figure A.3: Calculations of a transient vibration with a full time width. Top row $(\mathrm{a}, \mathrm{b})$ : large amplitude of vibration $\left(\Phi_{a}=2 \pi / 5\right)$. Middle row $(\mathrm{c}, \mathrm{d})$ : small amplitude of vibration $\left(\Phi_{a}=2 \pi / 50\right)$. Bottom row (e,f): very small amplitude of vibration $\left(\Phi_{a}=2 \pi / 500\right)$. The left column (a, c, e) shows the RF power spectra, where the bold lines show the shape of the non-linear gain used for the signal processing. The right column (b, d, f) shows a comparison between the exact vibration motion (solid line) and the vibration obtained after the signal processing (circles). All the calculations are made with the same feedback conditions $\left(R_{e}=10^{-7}\right.$ and $\left.\Omega_{0}=1.1 \Omega_{R}\right)$ and the same bandpass filter $\Delta \Omega_{0}=\Omega_{R} / 1.5$ (corresponding to the dashed vertical lines). (58)

induced by the modification of the carrier amplitude (77).

The images on the left column show that the frequency distance between the two maxima (located symmetrically on both side of the carrier frequency) decreases. Also, in it can be noted that the amplitude of the maximum located on the right side of the carrier frequency increases (77).

The right column of Figure A.3 shows that regardless of the shape of the non-linear gain, the impulse vibration is recovered with an accurate amplitude and an accurate time delay. Nevertheless, it can be noticed a small degradation of the signal-to-noise-ratio (roughly by a factor 2 ) when $\Omega_{0}=1.01 \Omega_{R}$. In Figure A.3f, the higher noise, which is visible at the base of the impulse vibration, can be attributed to the fact that the adjustment of the noise power 
Appendix A. Appendix: Non-linear laser dynamics induced by frequency shifted optical feedback

spectrum by the non-linear gain is not always perfect and that the amount of noise before the gain compensation inside the bandwidth filter increases when $\frac{\Omega_{0}}{\Omega_{R}} \rightarrow 1$ (see Figure A.3e).

\section{A.2}

\section{Gain of the nonlinear laser dynamics}

As explained in the previous section, vibration measurements without bias are possible with frequency shifted optical feedback in the laser, if the gain compensation is applied in the signal processing. So the gain of the laser dynamics needs to be known. The aim of the present section is to obtain an analytic expression of the complex gain, for all the feedback conditions and laser dynamics.

\section{A.2.1}

\section{Bifurcation analysis}

To analytically study the dynamical response of a laser subject to frequency shifted optical feedback $\left(R_{e} \neq 0\right)$, we perfomed a bifurcation analysis similar to those described in $(77,78,79)$. We focus our analysis on the case $\Omega_{0} \approx \Omega_{R}$, where $\Omega_{R}$ is the intrinsic relaxation oscillation frequency of the laser. For a more convenient theoretical analysis, we have reformulated the set of equations A-1 by introducing the new variables $\mathrm{x}$ and $\mathrm{y}$ which are, respectively, the deviation from the nonzero intensity laser steady-state and the population inversion steady-state. We also introduce the new modulation parameters $\delta_{e}, \sigma_{e}$, defined by :

$$
\begin{array}{r}
I=I_{S}(1+y), N=N_{S}\left(1+\frac{\Omega_{R}}{\gamma_{c}} x\right), s=\Omega_{R} t, \\
\sigma_{e}=\frac{\Omega_{0}}{\Omega_{R}}, \delta_{e}=2 \sqrt{R_{e}} \frac{\gamma_{c}}{\Omega_{R}}
\end{array}
$$

In the absence of noise, the set of equations A-1 can then be rewritten as $(77,78,79)$ :

$$
\begin{gathered}
\frac{d x}{d s}=y-\epsilon x[1+(\eta-1)(1+y)], \\
\frac{d y}{d s}=(1+y)\left[x+\delta_{e} \cos \left(\sigma_{e} s+\Phi_{e}\right)\right] .
\end{gathered}
$$

For our microchip laser, $\epsilon=\gamma_{1} / \Omega_{R} \approx 10^{-3}$ is a small quantity which motivates an asymptotic analysis of equations A-14. For $\sigma_{e} \approx 1$, the leading approximation of the solutions for the deviations $\mathrm{x}$ and $\mathrm{y}$ from the nonzero 
Appendix A. Appendix: Non-linear laser dynamics induced by frequency shifted optical feedback

intensity state can be written as $(77,78,79)$ :

$$
\begin{array}{r}
x=A \exp (i S)-i \frac{A^{2}}{3} \exp (i 2 S)+c c, \\
y=-i A \exp (i S)-\frac{2}{3} A^{2} \exp (i 2 S)+c c,
\end{array}
$$

where $S=\sigma_{e} s=\Omega_{0} t$. The complex amplitude $A=0\left(\epsilon^{1 / 2}\right)$ of the small periodic oscillation is a function of the slow time $\epsilon s$ and satisfies the following equation (see $(78,79)$ for more details):

$$
\frac{d A}{d s}=-i\left[\sigma_{e}-1\right] A-i \frac{A^{2} A^{*}}{6}-\frac{\eta \epsilon}{2} A+i \frac{\delta_{e}}{4} \exp \left(i \Phi_{e}\right) .
$$

Note that our asymptotic approximation is valid when $\epsilon \rightarrow 0$, assuming that $\sqrt{R_{e}}$ and $\left|\sigma_{e}-1\right|$ are at maximum $0(\epsilon)$ quantities.

In the right-hand side of equations A-15a A-15b, the first terms correspond to the output power modulation at the modulation frequency $F_{0}$. The second terms correspond to the first harmonic modulation induced by the nonlinear coupling between the laser intensity and the laser population inversion through the stimulated emission.

\section{A. 2.2}

\section{Asymptotic solution for the nonlinear gain}

To obtain the gain (the modulation transfer function) of the laser dynamic, we now investigate the solutions of Equation A-16, in the particular case of a vibrating target with a harmonic oscillation:

$$
\Phi_{e}(s)=\Phi_{0}+\Phi_{a} \sin \left(\sigma_{a} s+\phi_{a}\right)
$$

where $\sigma_{a}=\frac{\Omega_{a}}{\Omega_{R}}$ is the normalized acoustic vibration frequency, $\Phi_{a}=\frac{2 \pi}{\lambda} 2 d_{a}$ is the phase-shift linked to the vibration amplitude $d_{a}, \phi_{a}$ the modulation phase shift and $\Phi_{0}$ an additional phase shift corresponding to the mean distance between the laser and the vibrating target. Without loss of generality, we assume that $\Phi_{0}=2 \pi m$, where $\mathrm{m}$ is an integer.

In the case of sub-wavelength vibration amplitudes, with $\Phi_{a} \ll 2 \pi$, eq. (A-16) can be rewritten as:

$$
\begin{aligned}
\frac{d A}{d s} & =-i\left[\sigma_{e}-1\right] A-i \frac{A^{2} A^{*}}{6}-\frac{\eta \epsilon}{2} A \\
& +i \frac{\delta_{e}}{4}\left[J_{0}\left(\Phi_{a}\right)+J_{1}\left(\Phi_{a}\right) \exp \left[i\left(\sigma_{a} s+\phi_{a}\right)\right]+J_{-1}\left(\Phi_{a}\right) \exp \left[i\left(-\sigma_{a} s-\phi_{a}\right)\right]\right]
\end{aligned}
$$

where $J_{n}\left(\Phi_{a}\right)$ is the Bessel function of the first kind and of order $\mathrm{n}$ with: 
Appendix A. Appendix: Non-linear laser dynamics induced by frequency shifted optical feedback

$J_{0}\left(\Phi_{a}\right) \approx 1, J_{1}\left(\Phi_{a}\right) \approx \Phi_{a} / 2$ and $J_{-1}\left(\Phi_{a}\right)=-J_{1}\left(\Phi_{a}\right)$.

The key term here is $-i \frac{A^{2} A^{*}}{6}$, which couples the amplitude of the modulation at the carrier frequency with the vibration amplitudes at the acoustic sideband frequencies $\left(\Omega_{0} \pm \Omega_{a}\right)$.

The solution of equation A-18 can be written as:

$$
A(s)=C_{e}+T_{R}(s)+R_{a} \exp \left[i\left(\sigma_{a} s+\phi_{a}\right)\right]+L_{a} \exp \left[-i\left(\sigma_{a} s+\phi_{a}\right)\right]
$$

where $C_{e}$ is the stationary modulation amplitude at the carrier frequency, $T_{R}(s)$ is the transient dynamics of random excitation around the stationary solution due to quantum noise, while $R_{a}$ and $L_{a}$ are respectively the amplitudes of the right and left sidebands of the acoustic vibration. Next, we will assume that the carrier signal is much stronger than the laser quantum noise and the vibration sidebands.

Keeping only the first order non-linear terms, Equation A-19 gives:

$$
\begin{aligned}
A^{2} A^{*} \approx & C_{e} C_{e} C_{e}^{*}+2 C_{e} C_{e}^{*} T_{R}+C_{e} C_{e} T_{R}^{*}+2 C_{e} C_{e}^{*} R_{a} \exp \left[i\left(\sigma_{a} s+\phi_{a}\right)\right] \\
& +C_{e} C_{e} R_{a}^{*} \exp \left[-i\left(\sigma_{a} s+\phi_{a}\right)\right]+2 C_{e} C_{e}^{*} L_{a} \exp \left[-i\left(\sigma_{a} s+\phi_{a}\right)\right] \\
& +C_{e} C_{e} L_{a}^{*} \exp \left[i\left(\sigma_{a} s+\phi_{a}\right)\right] .
\end{aligned}
$$

Applying equations A-19 and A-20 into eq. (A-18) gives the following equalities:

$$
\begin{aligned}
i\left(\sigma_{e}-1\right) C_{e}+i \frac{C_{e}^{2} C_{e}^{*}}{6}+\frac{\eta \epsilon}{2} C_{e} & =i \frac{\delta_{e}}{4} J_{0}\left(\Phi_{a}\right), \\
\frac{d T_{R}}{d s}=-\left[\frac{\eta \epsilon}{2}+i\left(\sigma_{e}-1\right)+i \frac{C_{e} C_{e}^{*}}{3}\right] T_{R}-i \frac{C_{e}^{2}}{6} T_{R}^{*} & \\
R_{a}\left[\frac{\eta \epsilon}{2}+i \sigma_{a}+i\left(\sigma_{e}-1\right)+i \frac{C_{e} C_{e}^{*}}{3}\right]+L_{a}^{*}\left[i \frac{C_{e} C_{e}}{6}\right] & =i \frac{\delta_{e}}{4} J_{1}\left(\Phi_{a}\right), \\
R_{a}^{*}\left[i \frac{C_{e} C_{e}}{6}\right]+L_{a}\left[\frac{\eta \epsilon}{2}-i \sigma_{a}+i\left(\sigma_{e}-1\right)+i \frac{C_{e} C_{e}^{*}}{3}\right] & =i \frac{\delta_{e}}{4} J_{-1}\left(\Phi_{a}\right) .
\end{aligned}
$$

Let us recall that the analytical resolution of Equation A-21a shows that the power spectrum of the output power modulation at the carrier frequency $\left(\left|C_{e}\left(\delta_{e}, \sigma_{e}\right)\right|^{2}\right.$ versus $\left.\sigma_{e}\right)$ exhibits hysteresis in the strong feedback situation and that the extent of the hysteresis zone increases with the amount of optical feedback $\sigma_{e}(77,78,80,81)$. Combined, the study of the transient dynamics (Equation A-21b) allows us to determine the noise power spectrum of the laser submitted to frequency shifted optical feedback.

It is also important to recall that the noise power spectrum is composed of two resonant curves with a resonant width $\eta \epsilon$ and with resonance frequencies 
Appendix A. Appendix: Non-linear laser dynamics induced by frequency shifted optical feedback

symmetrically located on both side of the carrier frequency (77):

$$
\begin{aligned}
\sigma_{R \pm}\left(\delta_{e}, \sigma_{e}\right. & \left.=\sigma_{e}\right) \pm \delta_{R}\left(\delta_{e}, \sigma_{e}\right), \\
\delta_{R}\left(\delta_{e}, \sigma_{e}\right) & =\sqrt{\left(\sigma_{e}-1+\frac{\left|C_{e}\left(\delta_{e}, \sigma_{e}\right)\right|^{2}}{3}\right)^{2}-\left(\frac{\left|C_{e}\left(\delta_{e}, \sigma_{e}\right)\right|^{2}}{6}\right)^{2}} .
\end{aligned}
$$

Equation A-22 shows that the modification of the noise power spectrum is linked to the strength of the optical feedback, specially through its dependence on the non-linear modulation amplitude .

For the vibration, equations A-21c and A-21d clearly show that $R_{a}$ and $L_{a}$ are coupled through the non-linear modulation amplitude $C_{e}$. Nevertheless, the resolution of equations A-21c and A-21d is straightforward and gives the following solutions for the modulation amplitude of the two sidebands:

$$
\begin{aligned}
& R_{a}\left(\sigma_{a}, \delta_{e}, \sigma_{e}\right)= \\
& \quad \frac{i \frac{\delta_{e}}{4} J_{1}\left(\Phi_{a}\right) B^{*}-\left(i \frac{\delta_{e}}{4} J_{-1}\left(\Phi_{a}\right)\right)^{*}\left[i \frac{\left|C_{e}\left(\delta_{e}, \sigma_{e}\right)\right|^{2}}{6}\right]}{A B-\left[\frac{\left|C_{e}\left(\delta_{e}, \sigma_{e}\right)\right|^{2}}{6}\right]}, \\
& L_{a}\left(\sigma_{a}, \delta_{e}, \sigma_{e}\right)= \\
& \frac{i \frac{\delta_{e}}{4} J_{-1}\left(\Phi_{a}\right) A^{*}-\left(i \frac{\delta_{e}}{4} J_{-1}\left(\Phi_{a}\right)\right)^{*}\left[i \frac{\left|C_{e}\left(\delta_{e}, \sigma_{e}\right)\right|^{2}}{6}\right]}{A B-\left[\frac{\left|C_{e}\left(\delta_{e}, \sigma_{e}\right)\right|^{2}}{6}\right]}
\end{aligned}
$$

where

$$
\begin{aligned}
& A=\left[\frac{\eta \epsilon}{2}+i \sigma_{a}+i\left(\sigma_{e}-1\right)+i \frac{\left|C_{e}\left(\delta_{e}, \sigma_{e}\right)\right|^{2}}{3}\right], \\
& B=\left[\frac{\eta \epsilon}{2}-i \sigma_{a}+i\left(\sigma_{e}-1\right)+i \frac{\left|C_{e}\left(\delta_{e}, \sigma_{e}\right)\right|^{2}}{3}\right] .
\end{aligned}
$$

We now follow by explaining why it is possible to extract an accurate value of $\Phi_{a}$ and, finally, of the vibration amplitude $d_{a}$, from the measurement of the two vibration sidebands $R_{a}$ and $L_{a}$ along with the carrier modulation $C_{e}$.

Using the two vibration sidebands given by equations A-23a and A-23b, we define the nonlinear gain of the laser dynamics by the following equation:

$$
\left.G \sigma_{v}, \delta_{e}, \sigma_{e}\right)= \begin{cases}R_{a}\left(\sigma_{v}-\sigma_{e}, \delta_{e}, \sigma_{e}\right) /\left[J_{1}\left(\Phi_{a}\right) \sqrt{R_{e}}\right] & \text { if } \sigma_{v} \geq \sigma_{e} \\ L_{a}\left(\sigma_{v}-\sigma_{e}, \delta_{e}, \sigma_{e}\right) /\left[J_{1}\left(\Phi_{a}\right) \sqrt{R_{e}}\right] & \text { if } \sigma_{v}<\sigma_{e}\end{cases}
$$

Like the noise power spectrum, this gain exhibits two resonance frequencies symmetrically located on both side of the carrier frequency (equations 
Appendix A. Appendix: Non-linear laser dynamics induced by frequency shifted optical feedback

A-22a and A-22b). This double resonance is due to the cross coupling between the vibration sidebands.

The frequency distance and the amplitude ratio between the two maxima depend on the feedback conditions and are roughly given by (77):

$$
\begin{array}{r}
2 \delta_{R}\left(\delta_{e}, \sigma_{e}\right) \approx 2\left[\sigma_{e}-1+\frac{\left|C_{e}\left(\delta_{e}, \sigma_{e}\right)\right|^{2}}{3}\right] \\
\frac{\left|G\left(\sigma_{e}+\delta_{R}, \delta_{e}, \sigma_{e}\right)\right|}{\left|G\left(\sigma_{e}-\delta_{R}, \delta_{e}, \sigma_{e}\right)\right|} \approx \frac{\left|C_{e}\left(\delta_{e}, \sigma_{e}\right)\right|^{2} / 6}{2 \delta_{R}\left(\delta_{e}, \sigma_{e}\right)} .
\end{array}
$$

In agreement with the results shown on Figure A.1a and b, Equation A25b explains that the amplitude of the two maxima, located symmetrically on both side of the carrier frequency, are of the same order of magnitude for strong optical feedback, when $\left|C_{e}\left(\delta_{e}, \sigma_{e}\right)\right|^{2} \approx 1$. Also, the right maximum disappears for weak optical feedback $\left|C_{e}\left(\delta_{e}, \sigma_{e}\right)\right|^{2}<1$, leading to the conventional LOFI linear gain, with only one resonance frequency $(30,31,34)$ :

$$
\left|G\left(\sigma_{v}, \delta_{e}, \sigma_{e}\right)\right| \approx\left|G_{L}\left(\sigma_{v}\right)\right|=\frac{\frac{\delta_{e}}{4 \sqrt{R_{e}}}}{\sqrt{\left(\sigma_{v}-1\right)^{2}+\left(\frac{\eta \epsilon}{2}\right)^{2}}}=\frac{\gamma_{c}}{\sqrt{4\left(\Omega_{v}-\Omega_{R}\right)^{2}+\left(\eta \gamma_{1}\right)^{2}}} .
$$

At this point, it can be noticed that the linear gain depends only on the intrinsic dynamical parameters of the laser $\left(\gamma_{1}, \gamma_{c}\right.$ and $\left.\eta\right)$, while the non-linear gain also depends on the feedback conditions $\left(R_{e}\right.$ and $\left.\Omega_{0}\right)$.

Also, in agreement with the results shown on Figure A.3a, c and e, equations A-25a and A-25b explain why the frequency distances between the two maxima decreases and why the amplitude of the maximum located on the right side of the carrier frequency increases when $\sigma_{e}=\Omega_{0} / \Omega_{R} \rightarrow 1$.

\section{A.2.3}

\section{Gain compensation for vibration measurements}

Using equations A-21a A-23A.2.2, we can obtain:

$$
C_{e}\left(\delta_{e}, \sigma_{e}\right)=G\left(\left(\sigma_{v}=\sigma_{e}, \delta_{e}, \sigma_{e}\right) J_{0}\left(\Phi_{a}\right) \sqrt{R_{e}}\right.
$$

Equations A.2.2 and A-27 show that an accurate measurement of the vibration can be extract from the non-linear laser dynamics $\left(C_{e}, R_{a}\right.$, and $\left.L_{a}\right)$, if the non-linear amplification is compensated:

$$
\begin{aligned}
\frac{J_{1}\left(\Phi_{a}\right)}{J_{0}\left(\Phi_{a}\right)} & =\frac{R_{a}\left(\sigma_{a}, \delta_{e}, \sigma_{e}\right)}{C_{e}\left(\delta_{e}, \sigma_{e}\right)} \frac{G\left(\left(\sigma_{v}=\sigma_{e}, \delta_{e}, \sigma_{e}\right)\right.}{G\left(\sigma_{v}=\sigma_{e}+\sigma_{a}, \delta_{e}, \sigma_{e}\right)}, \\
\frac{J_{-1}\left(\Phi_{a}\right)}{J_{0}\left(\Phi_{a}\right)} & =\frac{L_{a}\left(\sigma_{a}, \delta_{e}, \sigma_{e}\right)}{C_{e}\left(\delta_{e}, \sigma_{e}\right)} \frac{G\left(\left(\sigma_{v}=\sigma_{e}, \delta_{e}, \sigma_{e}\right)\right.}{G\left(\sigma_{v}=\sigma_{e}-\sigma_{a}, \delta_{e}, \sigma_{e}\right)} .
\end{aligned}
$$


Appendix A. Appendix: Non-linear laser dynamics induced by frequency shifted optical feedback

At this point, knowing the accurate ratio between the Bessel functions, $\Phi_{a}$ could be determined.

For example, if we recall that for a very small vibration amplitude $\left(\Phi_{a} \ll\right.$ $2 \pi$ ) these ratios are equal to $J_{1}\left(\Phi_{a}\right) / J_{0}\left(\Phi_{a}\right) \approx \Phi_{a} / 2$ and $J_{-1}\left(\Phi_{a}\right) / J_{0}\left(\Phi_{a}\right) \approx$ $-\Phi_{a} / 2$. Also, as already mentioned earlier, equations A-28a A-28b show that the multiplication of $G\left(\sigma_{v}, \delta_{e}, \sigma_{e}\right)$ by any constant has no influence on the relative ratio between the amplitude of the spectral components of the vibration spectrum, the Bessel functions. Therefore, to restore accurate vibration measurement, the frequency shape of the complex gain is important, but not its exact value.

Figure A.4 shows a comparison between the non-linear gain $G\left(\sigma_{v}, \delta_{e}, \sigma_{e}\right)$ given by Equation A.2.2 and the amplitude of the vibration sidebands $\left(C_{e}, R_{a}\right.$ and $L_{a}$ ) obtained from a numerical simulation. Figure A.4a shows the RF power spectrum of the laser output power modulation for a strong optical feedback $\left(R_{e}=10^{-7}\right.$ and $\left.\Omega_{e}=1.1 \Omega_{R}\right)$ and a harmonic vibration $\left(\Omega_{a}=\Omega_{R} / 7\right)$ with a small amplitude of vibration. The signal is composed of one peak at the carrier frequency $\left(\sigma_{e}\right)$ and two peaks for the vibration sidebands $\left(\sigma_{e} \pm \sigma_{a}\right)$. The noise power spectrum exhibits a double resonance with resonance frequencies symmetrically located on both sides of the carrier frequency $\left(\sigma_{e} \pm \delta_{R}\right)$.

Also, Figure A.4a allows us to show the link between the noise power spectrum, the gain of the laser dynamics and the relative amplitudes of the spectral components of the vibration spectrum. To check the validity of equations A-28a and A-28b, the shape (i.e. not the absolute value) of the complex gain is simply determined by adjusting the shape of the analytical expression of $G\left(\sigma_{v}, \delta_{e}, \sigma_{e}\right)$ on the noise power spectrum (lower gain curve). In practice, specially the distance and the relative height between the two resonance peaks are carefully adjusted. Then, if the shape of the adjusted gain is renormalized (multiplied by a constant) to fit the modulation amplitude at the carrier frequency (upper gain curve), the division of the renormalized gain by $J_{0}\left(\Phi_{a}\right) / J_{1}\left(\Phi_{a}\right)$, allows us to obtains a very good agreement between the value of the gain and the amplitude of the vibration sidebands (middle gain curve).

This verification demonstrates that the frequency shapes of the complex gain, which must be known to restore accurate vibration measurement, can be simply determined experimentally by adjusting the shape of $G\left(\sigma_{v}, \delta_{e}, \sigma_{e}\right)$ on the shape of the noise power spectrum. This applies for both feedback conditions and, therefore, linear or nonlinear laser dynamics.

Plots b) and c) show the amplitude and the phase of the vibration sidebands obtained for different modulation frequencies $\left(\sigma_{a}\right)$. From these 
Appendix A. Appendix: Non-linear laser dynamics induced by frequency shifted optical feedback

a) RF Power Spectrum (dB)

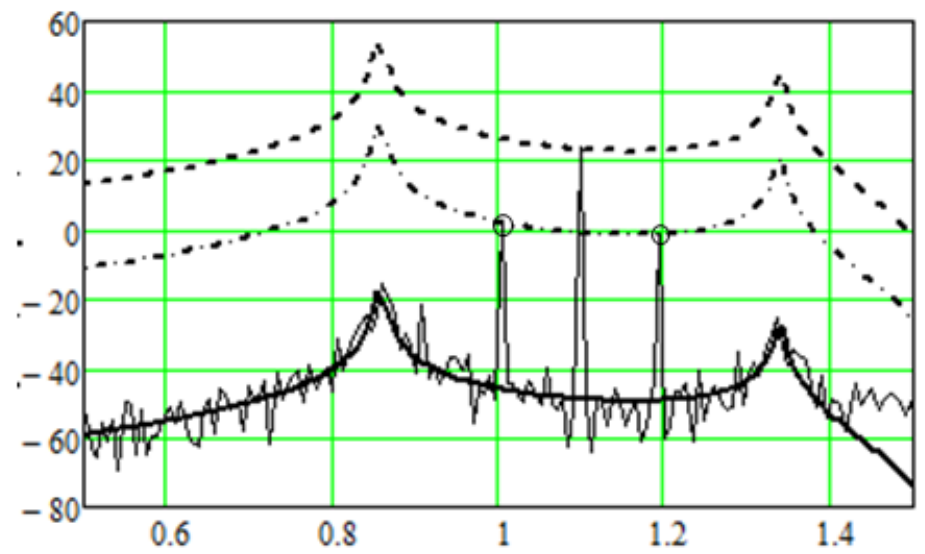

b) Sidebands amplitude (dB)

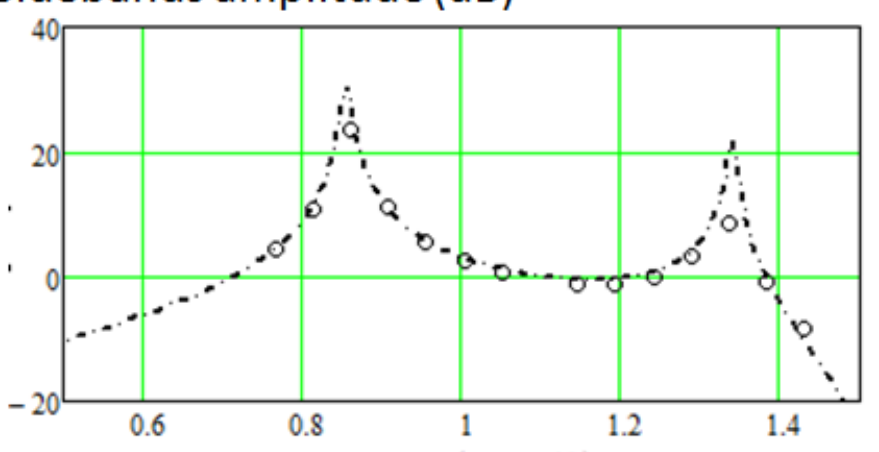

c) Sidebands phase $/ \pi$

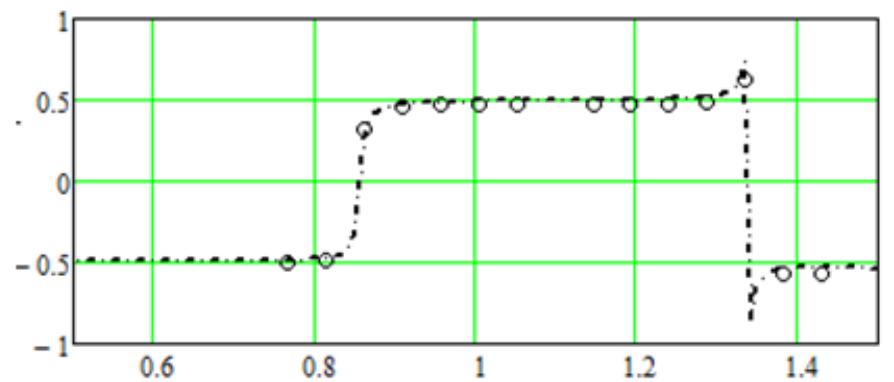

Normalized Frequency : $F / F_{R}$

Figure A.4: Comparison between the complex amplitude of the vibration sidebands (circle) obtained from a numerical simulation and the non-linear gain (solid, dashed and dash-dotted lines) obtained analytically. a) RF power spectrum of the laser output power modulation for a strong optical feedback $\left(R_{e}=10^{-7}\right.$ and $\left.\Omega_{0}=1.1 \Omega_{R}\right)$ and a harmonic vibration $\left(\Omega_{0} \approx \Omega_{R} / 10\right)$ with a small amplitude of vibration $\Phi_{a}=2 \pi / 50$. b) Amplitude and c) phase of the vibration sidebands, for 7 acoustic frequencies. (58)

results it is possible to see a good agreement between the numerical results and the non-linear complex gain $G\left(\sigma_{v}, \delta_{e}, \sigma_{e}\right)$ previously determined, confirming the possibility to extract quantitative vibration measurements. 